TRANSACTIONS OF THE

AMERICAN MATHEMATICAL SOCIETY

Volume 349, Number 1, January 1997, Pages 1-54

S 0002-9947(97)01752-2

\title{
HOMOTOPY COHERENT CATEGORY THEORY
}

\author{
JEAN-MARC CORDIER AND TIMOTHY PORTER
}

\begin{abstract}
This article is an introduction to the categorical theory of homotopy coherence. It is based on the construction of the homotopy coherent analogues of end and coend, extending ideas of Meyer and others. The paper aims to develop homotopy coherent analogues of many of the results of elementary category theory, in particular it handles a homotopy coherent form of the Yoneda lemma and of Kan extensions. This latter area is linked with the theory of generalised derived functors.
\end{abstract}

In homotopy theory, one often needs to "do" universal algebra "up to homotopy" for instance in the theory of homotopy everything $H$-spaces. Universal algebra nowadays is most easily expressed in categorical terms, so this calls for a form of category theory "up to homotopy" (cf. Heller [34]).

In studying the homotopy theory of compact metric spaces, there is the classically known complication that the assignment of the nerve of an open cover to a cover is only a functor up to homotopy. Thus in shape theory, [39], one does not have a very rich underlying homotopy theory. Strong shape (cf. Lisica and Mardešić, [37]) and Steenrod homotopy (cf. Edwards and Hastings [27]) have a richer theory but at the cost of much harder proofs. Shape theory has been interpreted categorically in an elegant way. This provides an overview of most aspects of the theory, as well as the basic proobject formulation that it shares with étale homotopy and the theory of derived categories. Can one perform a similar process with strong shape and hence provide tools for enriching that theory, rigidifying étale homotopy and enriching derived categories? We note, in particular, Grothendieck's plan for the theory of derived categories, sketched out in 'Pursuing Stacks', [31], which bears an uncanny ressemblance to the view of homotopy theory put forward by Heller in [34].

Grothendieck's 'Pursuing Stacks' program, in fact, again raises the spectre of doing categorical construction 'up to homotopy', as his image of a stack is as a sheaf 'up to homotopy' in which the stalks are algebraic models of homotopy types and the whole object has geometric meaning.

Problems of homotopy coherence also arise naturally in studying equivariant homotopy theory. If $G$ is a group then the equivariant homotopy of $G$-complexes can be studied in a useful way by translating to a category of diagrams indexed by the orbit category of $G$, that is the full subcategory of $G$-sets determined by

Received by the editors July 24, 1995.

1991 Mathematics Subject Classification. Primary 18D20, 18D05, 18G30, 18A99.

Key words and phrases. Simplicially enriched categories, homotopy coherent ends and coends, Yoneda lemma.

(C)1997 American Mathematical Society 
the coset spaces $G / H$. If however $G$ is a topological group then this category is more naturally considered to be simplicially enriched and the equivariant homotopy theory of the $G$-complexes ends up benefitting from results on homotopy coherence to handle the bar-resolutions etc. used to translate from the equivariant setting to the category of diagrams; see [24] and the references it contains. Again the equivariant theory looks like the discrete case but with the categorical arguments done 'up to homotopy'.

In this paper we try to lay some of the foundations of such a theory of categories 'up to homotopy' or more exactly 'up to coherent homotopies'. The method we use is based on earlier work on:

1. simplicial descriptions of homotopy coherence, [16];

2. Vogt's theorem, [52], interpreting homotopy categories of diagrams as categories of coherent diagrams [21], see also [20] and more recently, [7];

3. rectifications of coherent diagrams, [18] and [23];

4. simplicial formulation of homotopy limits, [10] and [18];

5. descriptions of Steenrod homology, [19] and [27];

6. ideas independently developed by Heller [33] and others;

7. geometric constructions in strong shape theory, cf. [37] and [32], and of course,

8. category theory and enriched category theory.

We aim for a simplicially enriched category theory with a simplicially based $\infty$ laxification of the structure. The simplicially enriched categories will usually be locally weakly Kan or locally Kan, corresponding to a lax $\infty$-category or lax $\infty$ groupoid structure via their nerves. Using $\infty$-lax or homotopy coherent ends and coends generalising the formulation of homotopy limits of homotopy coherent diagrams given in [10] or [18], we develop further the theory of homotopy coherent Kan extensions (related to ideas of Heller in [33] and earlier work by other authors). This yields a homotopy coherent form of the Yoneda lemma, the 'revelation' of a homotopy associative composition on the simplicial sets of coherent transformations between simplicial functors, which satisfies a "Godement interchange rule" in a homotopy coherent way. Homotopy coherent forms of adjointness result as well as representability up to homotopy coherence for certain simplicially enriched functors, a result that generalises to some extent both the formal representability results of Bénabou (cf. Mac Lane [38]) and the Brown representability theorem from algebraic topology (cf. Spanier [47], Adams [1] etc). The detailed proofs of these latter results are not given in this article.

Some of the results in the early sections of this paper were already contained the preprints [17] and [22].

\section{Contents}

1. Simplicially coherent ends and coends 4

2. Coherent ends and Kan complexes 11

3. Coherent transformations and the coherent Yoneda lemma 14

4. Ordinal sum and composition of coherent transformations 21

5. Coherent extensions 30

6. Coherent extensions with locally Kan codomain 33

7. The coherent Godement law 36 
8. Universality results in a coherent setting 40

9. Evaluation of coherent morphisms 43

10. Coherent adjunctions $\quad 47$

References $\quad 52$

\section{ACKNOWLEDGEMENTS}

The main results of this research were sketched out during a visit of the second author funded by a Royal Society grant for a sabbatical term in Amiens and Paris, during the summer of 1987.

\section{Notation AND TERminology}

The basic category theory we will need can be found in Mac Lane, [38], especially the theory of ends and their link with Kan extensions. The theory of enriched categories, indexed limits, etc., can be found in Kelly, [36], or Gray's article, [30]. For simplicial techniques the reference we will most often use is Bousfield and Kan, [11].

This may seem a lot of prerequisites, so a brief explanation is perhaps called for. The use of ends and coends extends that of limits and colimits, which is well known amongst homotopy theorists. Ends are a type of limit for "functors of two variables", $T: \mathbf{A}^{o p} \times \mathbf{A} \rightarrow \mathbf{B}$. The best illustration is probably when $F, G: \mathbf{A} \rightarrow \mathbf{B}$ are two functors with $\mathbf{A}$ small; then set $T\left(A, A^{\prime}\right)=\mathbf{B}\left(F A, G A^{\prime}\right)$. The corresponding end $\int_{A} T(A, A)$ is the set of natural transformations from $F$ to $G$. It is constructed by 'averaging out' the action of $\mathbf{A}$ on both the left and right of the collection of sets $T\left(A, A^{\prime}\right)$, picking out the fixed points of this action. Dually a coend, $\int^{A} T(A, A)$, will correspond to the set of orbits of the action. Examples of coends include the geometric realisation functor giving $|X|$ for a simplicial set $X$ via a coend formula

$$
|X|=\int^{n} X_{n} \times \Delta^{n} \text {. }
$$

The main method used in this paper is to place ourselves in an enriched setting (see below), to develop homotopy end and coend constructions analogous to homotopy limits and colimits, and to combine them to get a homotopy coherent analogue of the natural transformations. Then we exploit this notion systematically attempting to obtain analogues of other 'well-loved' constructions of category theory. The particular form of enriched setting we use will be that of simplicially enriched categories.

The category of combinatorial simplices will be denoted $\boldsymbol{\Delta}$. The symbol, $S$, will denote the category of simplicial sets, so that $S=F u n c\left(\boldsymbol{\Delta}^{o p}\right.$, Sets $)$; Top will denote the category of compactly generated spaces and Top will denote the $S$-enriched category (or $S$-category) obtained by setting, for each $n \geq 0$,

$$
\operatorname{Top}(X, Y)_{n}=\operatorname{Top}\left(X \times \Delta^{n}, Y\right) .
$$

In general, if a category is considered both in an $S$-enriched form and without its $S$-enrichment, the notation will reflect this by using a bold type for the $S$-enriched version. Thus $\mathbf{B}$ is $S$-enriched, but $\mathcal{B}$ is simply a category. At some points, it is 
difficult to be entirely thorough with this convention due to interference with other notation, but this convention will in general be adopted throughout the paper.

If $A, B$ are objects in a general $S$-category $\mathbf{B}$, the simplicial hom-set corresponding to $(A, B)$ will be denoted $\mathbf{B}(A, B)$. In considering $\mathbf{S}$, the simplicially enriched category of simplicial sets, we have

$$
\mathbf{S}(X, Y)_{n}=S(X \times \Delta[n], Y) .
$$

(Here $\Delta[n]$ is the simplicial $n$-simplex, $\Delta[n]=\boldsymbol{\Delta}(,[n])$.)

If $K$ is a simplicial set and $B$ is an object of $\mathbf{B}$, the tensor of $B$ by $K, K \bar{\otimes} B$, if it exists, satisfies the universal property given by the isomorphism (natural in $B^{\prime}$ )

$$
\mathbf{B}\left(K \bar{\otimes} B, B^{\prime}\right) \cong \mathbf{S}\left(K, \mathbf{B}\left(B, B^{\prime}\right)\right) .
$$

The cotensor $\overline{\mathbf{B}}(K, B)$ is defined to be the object of $\mathbf{B}$, again if it exists, satisfying

$$
\mathbf{B}\left(B^{\prime}, \overline{\mathbf{B}}(K, B)\right) \cong \mathbf{S}\left(K, \mathbf{B}\left(B^{\prime}, B\right)\right),
$$

naturally in $B^{\prime}$. For more details on tensor and cotensors, see Quillen, [45], Gray, [30] or Kelly, [36]. For us a tensor $K \bar{\otimes} B$ replaces a product $K \times B$ when the ambient $S$-category, $\mathbf{B}$, is not that of simplicial sets. Dually the cotensor $\overline{\mathbf{B}}(K, B)$ replaces a 'mapping space' object. In particular we will need these with $K=\Delta[1]$, the 1simplex, giving a natural cylinder or cocylinder construction, and thus a homotopy theory. We also will need the case $K=(\Delta[1])^{n}$, the $n$-cube, so as to handle and internalise the homotopy coherence that is initially coded in the 'external' homobjects $\mathbf{B}(X, Y)$.

The subcategory of $\mathbf{S}$ determined by the Kan complexes will be denoted Kan and a general $S$-category $\mathbf{B}$ will be said to be locally Kan if for each pair, $(A, B)$, of objects of $\mathbf{B}, \mathbf{B}(A, B)$ is a Kan complex. If $\mathbf{B}$ is locally Kan, and is either tensored or cotensored then it is comparatively easy to develop a well-behaved homotopy theory in B (cf. Kamps and Porter [35]). Similarly "locally weakly Kan" will mean that the hom-objects are weak Kan complexes, that is, that fillers of zeroth or last face horns are not demanded in these hom-objects.

An unknown referee asked why locally weakly Kan categories were important. The motive for studying them is that whilst categories such as spaces, chain complexes, $\omega$-groupoids, crossed complexes, etc. are locally Kan, the category of $\infty$ categories would seem to be enriched over weak Kan simplicial sets having a nice canonical filler condition, discovered by J. E. Roberts, [43] and used by Street, [48], and Verity, [51], in their theory of Complicial Sets. When, as in recent work in topological quantum field theory, the objects encountered look like lax infinity categories, then experience with general Kan complexes, as against simplicial groups, suggests that the 'laxity' corresponds to non-canonical fillers. Thus locally weakly Kan categories seem to be one possible lax-analogue of $\infty$-categories and in part, our attempt here to mimic large chunks of basic category theory can be interpreted as a 'test bed' for the partial validation of such a hypothesis.

\section{Simplicially COHEREnT ENDS AND COEndS}

As pointed out above, end and coends are basic constructions in category theory. Mac Lane, [38], shows, for example, how the important notion of Kan extension can be encoded in the language of ends and coends and then that formulation can be used together with the 'end-calculus' and results such as 'Fubini's theorem', to give quick, elegant proofs of some of the important results in the development of that 
subject. In this section, we develop the basic machinery of simplicially coherent ends and coends in a general $S$-enriched category. Results that depend on the $S$-category being locally Kan will be postponed to section 2 .

Variants of these have been proposed by several authors (Segal, [46], Meyer, [41], Heller, [33], Dwyer, and Kan, [26] as well as Cordier, [18] and Cordier and Porter, [21]). They generalise homotopy limits, and as we will show, can be used with good effect to construct a homotopy coherent version of category theory.

Let $\mathbf{A}$ be a small $S$-category. For $A, B$ in $\mathbf{A}$, form the bisimplicial set $X(A, B)$ defined by

$$
X(A, B)_{n, \star}=\coprod_{A_{0} \ldots A_{n}} \mathbf{A}\left(A, A_{0}\right)_{\star} \times \mathbf{A}\left(A_{0}, A_{1}\right)_{\star} \times \ldots \times \mathbf{A}\left(A_{n}, B\right)_{\star}
$$

where

$$
d_{j}: X(A, B)_{n, \star} \rightarrow X(A, B)_{n-1, \star}
$$

is defined by composition in $\mathbf{A}$,

$$
\mathbf{A}\left(A_{i-1}, A_{i}\right) \times \mathbf{A}\left(A_{i}, A_{i+1}\right) \rightarrow \mathbf{A}\left(A_{i-1}, A_{i+1}\right)
$$

(we write $A_{-1}=A, A_{n+1}=B$ for the purposes of this definition), and $s_{i}$ : $X(A, B)_{n} \rightarrow X(A, B)_{n+1}$ is induced by the morphism,

$$
\Delta[0] \rightarrow \mathbf{A}\left(A_{i}, A_{i}\right)
$$

the simplicial morphism 'constant on $I d_{A_{i}}$ '.

Now set $\hat{\mathbf{A}}(A, B)=\operatorname{Diag} X(A, B)$, the diagonal simplicial set. We note that, in general, for a bisimplicial set, $X_{\bullet}, \star$,

$$
\operatorname{Diag} X_{\star} \cong \int^{[n]} \Delta[n] \times X_{n, \star} .
$$

Example. Let $\mathbf{A}$ be a small category, $A, B$ objects of $\mathbf{A}$. Consider $\mathbf{A}$ as an $S$ category with the trivial simplicial enrichment. Let $A \downarrow \mathbf{A} \downarrow B$ be the category of objects under $A$ and over $B$; then $X(A, B) \cong \operatorname{Ner}(A \downarrow \mathbf{A} \downarrow B)$, the nerve of this category.

This example suggests the use we will make of the $X(A, B)$, especially if one considers the use of the comma categories $\mathbf{A} \downarrow A$ by Bousfield and Kan, [11], and the extension to $S$-enriched indexing categories given by Bourn and the first author, [10].

Definition 1.1. Let $\mathbf{B}$ be a complete $S$-category, $\mathbf{A}$, as above, a (small) $S$-category and $T: \mathbf{A}^{o p} \times \mathbf{A} \rightarrow \mathbf{B}$, an $S$-functor.

The simplicially coherent end of $T$ will be the object $\oint_{A} T(A, A)$ of $\mathbf{B}$ defined by

$$
\oint_{A} T(A, A)=\int_{\left(A^{\prime}, A^{\prime \prime}\right)} \overline{\mathbf{B}}\left(\hat{\mathbf{A}}\left(A^{\prime}, A^{\prime \prime}\right), T\left(A^{\prime}, A^{\prime \prime}\right)\right)
$$

where $\left(A^{\prime}, A^{\prime \prime}\right) \in \mathbf{A}^{o p} \times \mathbf{A}$.

Remark. We will be consistently using $\hat{\mathbf{A}}$ to index these coherent ends; however it is important to note that if we were to replace $X(A, B)$ by $X(A, B)^{*}$ where $d_{i}^{n *}=d_{n-i}^{n}, s_{i}^{n *}=s_{n-i}^{n}$, we would obtain a parallel theory. 
Given any cosimplicial object $Y$ in $\mathbf{B}$ (so $Y: \Delta \rightarrow \mathbf{B}$ ), we can form the total object of $Y$ in $\mathbf{B}$ defined by

$$
\int_{[n]} \overline{\mathbf{B}}\left(\boldsymbol{\Delta}[n], Y^{n}\right)
$$

Thus in the case when $\mathbf{B}=\mathbf{S}, Y$ is a cosimplicial simplicial set and the total complex, denoted $\operatorname{Tot}(Y)$ or hom $(\Delta, Y)$, introduced by Bousfield and Kan [11], is $\int_{[n]} \mathbf{S}\left(\Delta[n], Y^{n}\right)$. This construction was used together with a cosimplicial replacement formula to provide a useful reformulation of the homotopy limit functor in [10]. Here we will give a similar description of $\oint_{A} T(A, A)$. Again let $T: \mathbf{A}^{o p} \times \mathbf{A} \rightarrow \mathbf{B}$ and set

$$
Y(T)^{n}=\prod_{A_{0}, \ldots, A_{n}} \overline{\mathbf{B}}\left(\mathbf{A}\left(A_{0}, A_{1}\right) \times \ldots \times \mathbf{A}\left(A_{n-1}, A_{n}\right), T\left(A_{0}, A_{n}\right)\right) .
$$

These form the basis for the cosimplicial object, $Y(T)$, where the coface and codegeneracy maps of $Y(T)$ are defined as follows: First let

$$
p_{A_{0}, \ldots, A_{n}}: Y(T)^{n} \rightarrow \overline{\mathbf{B}}\left(\mathbf{A}\left(A_{0}, A_{1}\right) \times \ldots \times \mathbf{A}\left(A_{n-1}, A_{n}\right), T\left(A_{0}, A_{n}\right)\right)
$$

denote the projection;

- for $0<i<n, c_{i}$ will denote the map from

$$
\mathbf{A}\left(A_{0}, A_{1}\right) \times \ldots \times \mathbf{A}\left(A_{i-1}, A_{i}\right) \times \mathbf{A}\left(A_{i}, A_{i+1}\right) \times \ldots \times \mathbf{A}\left(A_{n-1}, A_{n}\right)
$$

to

$$
\mathbf{A}\left(A_{0}, A_{1}\right) \times \ldots \times \mathbf{A}\left(A_{i-1}, A_{i+1}\right) \times \ldots \times \mathbf{A}\left(A_{n-1}, A_{n}\right)
$$

induced by the composition from $\mathbf{A}\left(A_{i-1}, A_{i}\right) \times \mathbf{A}\left(A_{i}, A_{i+1}\right)$ to $\mathbf{A}\left(A_{i-1}, A_{i+1}\right)$;

- for $0 \leq i \leq n-1, k_{i}$ will denote the map

$$
\begin{aligned}
\mathbf{A}\left(A_{0}, A_{1}\right) \times \ldots \times \mathbf{A}\left(A_{n-2}, A_{n-1}\right) & \\
& \rightarrow \mathbf{A}\left(A_{0}, A_{1}\right) \times \ldots \times \mathbf{A}\left(A_{i}, A_{i}\right) \times \ldots \times \mathbf{A}\left(A_{n-2}, A_{n-1}\right)
\end{aligned}
$$

induced by the map $\Delta[0] \rightarrow \mathbf{A}\left(A_{i}, A_{i}\right)$ which picks out the identity map, (i.e. the "name of the identity map");

- for any objects $A, A^{\prime}, A^{\prime \prime}$;

$$
T(, A)_{A^{\prime}, A^{\prime \prime}}: \mathbf{A}\left(A^{\prime}, A^{\prime \prime}\right) \rightarrow \mathbf{B}\left(T\left(A^{\prime \prime}, A\right), T\left(A^{\prime}, A\right)\right)
$$

and

$$
T(A,)_{A^{\prime}, A^{\prime \prime}}: \mathbf{A}\left(A^{\prime}, A^{\prime \prime}\right) \rightarrow \mathbf{B}\left(T\left(A, A^{\prime}\right), T\left(A, A^{\prime \prime}\right)\right)
$$

will denote the maps corresponding to the fact that $T(, A)$ and $T(A$,$) are$ S-functors.

Now the codegeneracies $s^{i}$ of $Y(T)$ are given by

$$
p_{A_{0}, \ldots, A_{n-1}} s^{i}=\overline{\mathbf{B}}\left(k_{i}, T\left(A_{0}, A_{n-1}\right)\right) p_{A_{0}, \ldots, A_{i}, A_{i}, \ldots, A_{n-2}}, \quad \text { for } 0 \leq i \leq n-1,
$$

whilst for $0<i<n$, the coface map $d^{i}$ is given by

$$
p_{A_{0}, \ldots, A_{n}} d^{i}=\overline{\mathbf{B}}\left(c_{i}, T\left(A_{0}, A_{n-1}\right)\right) p_{A_{0}, \ldots, A_{i-1}, A_{i+1}, \ldots, A_{n}}
$$

(i.e. $s^{i}$ and $d^{i}$ are induced by the inclusion of identities and the composition in the usual way; the slightly more complicated formulation is forced on us by the 
setting). This leaves $d^{0}$ and $d^{n}$. These are slightly more difficult to specify. The map $T(-, A)_{A^{\prime}, A^{\prime \prime}}: \mathbf{A}\left(A^{\prime}, A^{\prime \prime}\right) \rightarrow \mathbf{B}\left(T\left(A^{\prime \prime}, A\right), T\left(A^{\prime}, A\right)\right)$ gives an element in

$$
\mathbf{S}\left(\mathbf{A}\left(A^{\prime}, A^{\prime \prime}\right), \mathbf{B}\left(T\left(A^{\prime \prime}, A\right), T\left(A^{\prime}, A\right)\right)\right)_{0}
$$

and hence in

$$
\mathbf{B}\left(T\left(A^{\prime \prime}, A\right), \overline{\mathbf{B}}\left(\mathbf{A}\left(A^{\prime}, A^{\prime \prime}\right), T\left(A^{\prime}, A\right)\right)\right)_{0}
$$

(as, by assumption, $\mathbf{B}$ is cotensored). This is thus a map

$$
T(, A)_{A^{\prime}, A^{\prime \prime}}: T\left(A^{\prime \prime}, A\right) \rightarrow \overline{\mathbf{B}}\left(\mathbf{A}\left(A^{\prime}, A^{\prime \prime}\right), T\left(A^{\prime}, A\right)\right)_{0} .
$$

We also need the isomorphism

$$
\begin{aligned}
\tau: \overline{\mathbf{B}}\left(\mathbf{A}\left(A_{0}, A_{1}\right) \times \ldots \times \mathbf{A}\left(A_{n-1}, A_{n}\right), T\left(A_{0}, A_{n}\right)\right) \\
\quad \rightarrow \overline{\mathbf{B}}\left(\mathbf{A}\left(A_{1}, A_{2}\right) \times \ldots \times \mathbf{A}\left(A_{n-1}, A_{n}\right), \overline{\mathbf{B}}\left(\mathbf{A}\left(A_{0}, A_{1}\right), T\left(A_{0}, A_{n}\right)\right)\right) .
\end{aligned}
$$

Define $d^{0}$ by $p_{A_{0}, \ldots, A_{n}} d^{0}=\tau \circ \overline{\mathbf{B}}\left(\mathbf{A}\left(A_{1}, A_{2}\right) \times \ldots \times \mathbf{A}\left(A_{n-1}, A_{n}\right), T\left(, A_{n}\right)_{A_{0}, A_{1}}\right)$. Similarly $T(A,)_{A^{\prime}, A^{\prime \prime}}: \mathbf{A}\left(A^{\prime}, A^{\prime \prime}\right) \rightarrow \mathbf{B}\left(T\left(A, A^{\prime}\right), T\left(A, A^{\prime \prime}\right)\right)$ gives us

$$
T(A,)_{A^{\prime}, A^{\prime \prime}}: T\left(A, A^{\prime}\right) \rightarrow \overline{\mathbf{B}}\left(\mathbf{A}\left(A^{\prime}, A^{\prime \prime}\right), T\left(A, A^{\prime \prime}\right)\right),
$$

and

$$
p_{A_{0}, \ldots, A_{n}} d^{n}=\tau^{\prime} \circ T\left(A_{0},\right)_{A_{n-1}, A_{n}},
$$

where $\tau^{\prime}$ is the analogous isomorphism. In practice we pretend that $\tau$ and $\tau^{\prime}$ are identities so that

$$
\begin{gathered}
p_{A_{0}, \ldots, A_{n}} d^{n}=\overline{\mathbf{B}}\left(c_{0}, T\left(, A_{n}\right)_{A_{0}, A_{1}}\right) p_{A_{1}, \ldots, A_{n}}, \\
p_{A_{0}, \ldots, A_{n}} d^{n}=\overline{\mathbf{B}}\left(c_{n}, T\left(A_{0},\right)_{A_{n-1}, A_{n}}\right) p_{A_{0}, \ldots, A_{n-1}}
\end{gathered}
$$

where $c_{0}$ and $c_{n}$ are the obvious projections. Hence we have here a very similar formulation to that in the case of Bousfield and Kan's cosimplicial replacement functor.

Proposition 1.2 (Cosimplicial replacement). Let $T$ be an $\mathbf{S}$-functor from $\mathbf{A}^{o p} \times \mathbf{A}$ to a complete $S$-category $\mathbf{B}$. Then the simplicially coherent end of $T$ is isomorphic to the total object of the cosimplicial object $Y(T)$ and hence has the following universal property: for $n \in \mathbb{N}$, let $p_{n}: N=\oint_{A} T(A, A) \rightarrow \overline{\mathbf{B}}\left(\Delta[n], Y(T)^{n}\right)$ be the canonical projection. Then for $\mu:[n] \rightarrow[m] \in \boldsymbol{\Delta}$,

$$
\overline{\mathbf{B}}\left(\Delta[n], Y(T)^{\mu}\right) p_{n}=\overline{\mathbf{B}}\left(\Delta[\mu], Y(T)^{m}\right) p_{m}
$$

and if $N^{\prime}$ is in $\mathbf{B}$, and $q_{n}: N^{\prime} \rightarrow \overline{\mathbf{B}}\left(\Delta[n], Y(T)^{n}\right), n \in \mathbb{N}$, is a family of morphisms so that for each $\mu:[n] \rightarrow[m]$

$$
\overline{\mathbf{B}}\left(\Delta[n], Y(T)^{\mu}\right) q_{n}=\overline{\mathbf{B}}\left(\Delta[\mu], Y(T)^{m}\right) q_{m},
$$

then there is a unique morphism $b: N^{\prime} \rightarrow N$ in $\mathbf{B}$ such that for all $n, p_{n} b=q_{n}$.

Proof. We start by noting that by the usual construction of the enriched end applied to the functor from $\boldsymbol{\Delta}^{o p} \times \boldsymbol{\Delta}$ to $\mathbf{B}$ which assigns to $([n],[m])$ the object $\overline{\mathbf{B}}\left(\Delta[n], Y(T)^{m}\right)$, the total object of $Y(T)$ is the kernel of the pair of morphisms,

$$
\prod_{n} \overline{\mathbf{B}}\left(\Delta[n], Y(T)^{n}\right) \longrightarrow \prod_{\mu:[n] \rightarrow[m]} \overline{\mathbf{B}}\left(\Delta[n], Y(T)^{m}\right),
$$

given by $\overline{\mathbf{B}}\left(\Delta[n], Y(T)^{\mu}\right)$ and $\overline{\mathbf{B}}\left(\Delta[\mu], Y(T)^{m}\right)$. This implies that the universal property is immediate, once the first part is proved. This first part is however 
merely an exercise in the end calculus (using "Fubini" (cf. Mac Lane, [38]) several times):

$$
\begin{array}{rl}
\oint_{A} & T(A, A) \\
= & \int_{A^{\prime}} \int_{A^{\prime \prime}} \overline{\mathbf{B}}\left(\int^{n} \Delta[n] \times \coprod \mathbf{A}\left(A^{\prime}, A_{0}\right) \times \ldots \times \mathbf{A}\left(A_{n}, A^{\prime \prime}\right), T\left(A^{\prime}, A^{\prime \prime}\right)\right) \\
= & \int_{n} \int_{A^{\prime}} \int_{A^{\prime \prime}} \prod \overline{\mathbf{B}}\left(\Delta[n] \times \mathbf{A}\left(A^{\prime}, A_{0}\right) \times \ldots \times \mathbf{A}\left(A_{n-1}, A_{n}\right),\right. \\
= & \left.\int_{n} \int_{A^{\prime}} \prod \overline{\mathbf{B}}\left(\mathbf{A}\left(A_{n}, A^{\prime \prime}\right), T\left(A^{\prime}, A^{\prime \prime}\right)\right)\right) \\
\left.\int_{A^{\prime \prime}} \overline{\mathbf{B}}\left(\mathbf{A}\left(A_{n}, A^{\prime \prime}\right), T\left(A^{\prime}, A^{\prime \prime}\right)\right)\right)
\end{array}
$$

where the indexation of the (co)products is over all $A_{0}, \ldots, A_{n}$ in $\mathbf{A}$. The "enriched Yoneda lemma" (see Kelly, [36]) allows one to evaluate the last part of this end. (The classical Yoneda lemma tells one that given a functor $F: A \rightarrow$ Sets, the natural transformations from a 'hom-set' functor $A(a,-)$ to $F$ are in bijective correspondence with the elements of $F(a)$. This is easily encoded into the language of ends, and then the natural way of enriching this gives the following isomorphism in our context.)

$$
\int_{A^{\prime \prime}} \overline{\mathbf{B}}\left(\mathbf{A}\left(A_{n}, A^{\prime \prime}\right), T\left(A^{\prime}, A^{\prime \prime}\right)\right) \cong T\left(A^{\prime}, A_{n}\right) .
$$

Continuing we get

$$
\begin{aligned}
& \oint_{A} T(A, A) \\
\cong & \int_{n} \int_{A^{\prime}} \prod \overline{\mathbf{B}}\left(\Delta[n] \times \mathbf{A}\left(A^{\prime}, A_{0}\right) \times \ldots \times \mathbf{A}\left(A_{n-1}, A_{n}\right), T\left(A^{\prime}, A_{n}\right)\right) \\
\cong & \int_{n} \prod \overline{\mathbf{B}}\left(\Delta[n] \times \mathbf{A}\left(A_{0}, A_{1}\right) \times \ldots \times \mathbf{A}\left(A_{n-1}, A_{n}\right), \int_{A^{\prime}} \overline{\mathbf{B}}\left(\mathbf{A}\left(A^{\prime}, A_{0}\right),\left(T\left(A^{\prime}, A_{n}\right)\right)\right)\right) \\
\cong & \int_{n} \overline{\mathbf{B}}\left(\Delta[n], \prod \overline{\mathbf{B}}\left(\mathbf{A}\left(A_{0}, A_{1}\right) \times \ldots \times \mathbf{A}\left(A_{n-1}, A_{n}\right), T\left(A_{0}, A_{n}\right)\right)\right) \\
\cong & \int_{n} \overline{\mathbf{B}}\left(\Delta[n], Y(T)^{n}\right),
\end{aligned}
$$

as required.

Examples. 1. Let $\mathbf{A}$ be an $S$-category and $T: \mathbf{A}^{o p} \times \mathbf{A} \rightarrow \mathbf{S}$ an $S$-functor. Then $\oint_{A} T(A, A)=\operatorname{Tot}(Y(T))$ in the sense of Bousfield and Kan, i.e. $Y$ is "cosimplicial replacement".

One can handle the elements of $\oint_{A} T(A, A)$ as follows, at least in low dimensions: if $\sigma \in Y(T)_{n}^{0}$, then $p(A) \sigma \in \mathbf{S}(\Delta[0], T(A, A))_{n}=S(\Delta[n], T(A, A))$ and so can be represented by a map from $\Delta[n]$ to $T(A, A)$. The coface $d^{0} \sigma \in Y(T)_{n}^{1}$ projects via $p\left(A_{0}, A_{1}\right)$ to an element in

$$
\mathbf{S}\left(\mathbf{A}\left(A_{0}, A_{1}\right), T\left(A_{0}, A_{1}\right)\right)_{n}
$$


thus is represented by some map

$$
p\left(A_{0}, A_{1}\right) d^{0} \sigma: \mathbf{A}\left(A_{0}, A_{1}\right) \times \Delta[n] \rightarrow T\left(A_{0}, A_{1}\right) .
$$

This, if one interprets the general coface formula above, is the composite

$$
\mathbf{A}\left(A_{0}, A_{1}\right) \times \Delta[n] \rightarrow \mathbf{A}\left(A_{0}, A_{1}\right) \times T\left(A_{1}, A_{1}\right) \rightarrow T\left(A_{0}, A_{1}\right),
$$

where the first map is $\mathbf{A}\left(A_{0}, A_{1}\right) \times p\left(A_{1}\right) \sigma$ and the second is the left action of $\mathbf{A}\left(A_{0}, A_{1}\right)$ on $T\left(A_{1}, A_{1}\right)$, i.e. "precomposition". Similarly for $p\left(A_{0}, A_{1}\right) d^{1} \sigma$ which thus is the composite

$$
\mathbf{A}\left(A_{0}, A_{1}\right) \times \Delta[n] \rightarrow \mathbf{A}\left(A_{0}, A_{1}\right) \times T\left(A_{0}, A_{0}\right) \rightarrow T\left(A_{0}, A_{1}\right)
$$

involving $\mathbf{A}\left(A_{0}, A_{1}\right) \times p\left(A_{0}\right) \sigma$ and the "post composition" map or right action.

With longer strings $A_{0}, A_{1}, \ldots, A_{n}$, the intermediate cofaces cause no problems and, following the detailed description given earlier, it is easy to write down $d^{i} \sigma$ for all $\sigma \in Y(T)_{n}^{m}$.

If $\sigma \in\left(\oint_{A} T(A, A)\right)_{n}$, then $\sigma: \Delta \times \Delta[n] \rightarrow Y(T)$. We will consider only $n=0$ and 1 , as it is only these that we will need in such detail later and this case is, here, quite typical of the general one. The extension to the general case poses no problems. Then $\sigma=\left(\sigma^{m}\right)$ where $\sigma^{m}: \Delta[m] \rightarrow Y(T)^{m}$ and for each $m$ and $0 \leq i \leq m, \sigma^{m} d^{i}=d^{i} \sigma^{m+1}$, similarly for degeneracies. We examine $\sigma^{1} d^{0}=d^{0} \sigma^{0}$ in detail. As both maps end up in $Y(T)^{1}$, we project via $p\left(A_{0}, A_{1}\right)$ and get that $p\left(A_{0}, A_{1}\right) d^{0} \sigma^{0}$ is as we have written earlier, whilst $p\left(A_{0}, A_{1}\right)\left(\sigma^{1} d^{0}\right)$ is

$$
\mathbf{A}\left(A_{0}, A_{1}\right) \rightarrow \mathbf{A}\left(A_{0}, A_{1}\right) \times \Delta[1] \stackrel{p\left(A_{0}, A_{1}\right) \sigma^{1}}{\rightarrow} T\left(A_{0}, A_{1}\right),
$$

where the first map is the inclusion into the top of the cylinder, i.e. interpreting $p\left(A_{0}, A_{1}\right) \sigma^{1} d^{0}$ as $d_{0}\left(p\left(A_{0}, A_{1}\right) \sigma^{1}\right)$. We have

$$
d_{0}\left(p\left(A_{0}, A_{1}\right) \sigma^{1}\right)=p\left(A_{0}, A_{1}\right) d^{0} \sigma^{0}
$$

and similarly

$$
d_{1}\left(p\left(A_{0}, A_{1}\right) \sigma^{1}\right)=p\left(A_{0}, A_{1}\right) d^{1} \sigma^{0}
$$

i.e. $p\left(A_{0}, A_{1}\right) \sigma^{1}$ is a homotopy between $p\left(A_{0}, A_{1}\right) d^{1} \sigma^{0}$ and $p\left(A_{0}, A_{1}\right) d^{0} \sigma^{0}$. We will return to this later in specific examples, for instance when we wish to interpret what it means for a collection of maps $\left(f_{A}: F(A) \rightarrow G(A)\right)$ to be a "coherent transformation".

2. Consider a 2-category $\mathbf{A}$ as an $S$-category by taking the nerve of each homcategory and let $T: \mathbf{A}^{o p} \times \mathbf{A} \rightarrow$ Cat be a 2-functor, again considered as being $S$-enriched rather than $C a t$-enriched. Then the above description of $\oint_{A} T(A, A)$ shows that this simplicially coherent end construction is isomorphic to the lax end construction of Bozapalides, [12], cf. [13].

3. Let $A$ be an ordinary small category considered as a trivially enriched $S$ category, and $T: A^{o p} \times A \rightarrow T$ op be a functor; then

$$
\oint_{A} T(A, A)=\int_{n} \prod \overline{T o p}\left(\Delta^{n}, T\left(A_{0}, A_{n}\right)\right),
$$

where the product is over all $n$-simplices, $A \stackrel{f_{1}}{\rightarrow} A \rightarrow \cdots \stackrel{f_{n}}{\rightarrow} A_{n}$ in $\operatorname{Ner} A$.

This sort of construction is well known in special cases, cf. Cordier, [18] or Vogt, [52], and interprets geometrically as saying that the elements of $\left(\oint_{A} T(A, A)\right)_{0}$ are the families of functions

$$
h\left(f_{1}, \ldots, f_{n}\right): \Delta^{n} \rightarrow T\left(A_{0}, A_{n}\right)
$$


such that, on writing a point in $\Delta^{n}$ as an $n$-tuple $\left(v_{1}, \ldots, v_{n}\right)$ with

$$
0 \leq v_{1} \leq \ldots \leq v_{n} \leq 1
$$

we have:

$$
\begin{aligned}
& h\left(f_{1}, \ldots, f_{n}\right)\left(v_{1} \leq \ldots \leq v_{n}\right) \\
& \quad= \begin{cases}T\left(f_{1}, A_{n}\right) h\left(f_{2}, \ldots, f_{n}\right)\left(v_{2} \leq \ldots \leq v_{n}\right) & \text { if } v_{1}=0 \\
h\left(f_{1}, \ldots, f_{i+1} f_{i}, \ldots, f_{n}\right)\left(v_{1} \leq \ldots v_{i} \leq v_{i+2} \leq \ldots \leq v_{n}\right) & \text { if } v_{i}=v_{i+1} \\
T\left(A_{0}, f_{n}\right) h\left(f_{1}, \ldots, f_{n-1}\right)\left(v_{1} \leq \ldots \leq v_{n-1}\right) & \text { if } v_{n}=1 \\
h\left(f_{1}, \ldots, f_{i-1}, f_{i+1}, \ldots, f_{n}\right)\left(v_{1} \leq \ldots \leq v_{i-1} \leq v_{i+1} \leq \ldots v_{n}\right) & \text { if } f_{i}=i d .\end{cases}
\end{aligned}
$$

This can be interpreted geometrically if $T\left(A, A^{\prime}\right)$ is $\operatorname{Top}\left(F A, G A^{\prime}\right)$, for instance.

Definition 1.3. Let $\mathbf{A}$ be an $S$-category and $T$ an $S$-functor from $\mathbf{A}^{o p} \times \mathbf{A}$ to a cocomplete $S$-category $\mathbf{B}$, tensored by $-\bar{\otimes}-: \mathbf{S} \times \mathbf{B} \rightarrow \mathbf{B}$.

The simplicially coherent coend of $T$ will be the object

$$
\oint^{A} T(A, A)=\int^{A^{\prime}, A^{\prime \prime}}\left(\widehat{\mathbf{A}^{o p}}\right)\left(A^{\prime}, A^{\prime \prime}\right) \bar{\otimes} T\left(A^{\prime}, A^{\prime \prime}\right)
$$

where $\left(\widehat{\mathbf{A}^{o p}}\right)\left({ }_{-},-\right)$is the $S$-functor from $\mathbf{A} \times \mathbf{A}^{o p}$ to $\mathbf{S}$ given by $\left(\widehat{\mathbf{A}^{o p}}\right)\left(A^{\prime}, A^{\prime \prime}\right)=$ $\operatorname{Diag} X\left(A^{\prime \prime}, A^{\prime}\right)$.

Proposition 1.4 (dual form).

$$
\oint^{A} T(A, A)=\int^{n} \Delta[n] \bar{\otimes}\left(\left(\coprod \mathbf{A}\left(A_{0}, A_{1}\right) \times \ldots \times \mathbf{A}\left(A_{n-1}, A_{n}\right)\right) \bar{\otimes} T\left(A_{n}, A_{0}\right)\right)
$$

where as usual the indexation of the coproduct is over all $A_{0}, \ldots, A_{n}$ in $\boldsymbol{A}$.

Remarks. 1. As we already have noted, if $Y$ is a bisimplicial set, Diag $Y$ is given by

$$
(\operatorname{Diag} Y) \bullet=\int^{n} \Delta[n] \times Y_{n, \bullet}
$$

thus the formula given for $\oint^{A} T(A, A)$ by the above is a generalisation of the diagonal, applied to a simplicial object $Y(T)$ in $\mathbf{B}$, given by the term in brackets. If $\mathbf{B}=\mathbf{S}$, we retrieve a diagonal; if $\mathbf{B}=\mathbf{C a t}$ then we have a description similar to that of a lax coend and for $\mathbf{B}=$ Top, we get a description in terms of higher homotopy coherence data, since the tensor, $K \bar{\otimes} \_$, is the product with the geometric realisation, $|K| \times \ldots$. This can be compared with the cobar construction of May, [40], Elmendorf, [28], Meyer, [41], and others.

2. We note that our results on coherent ends and coends are formal in the sense that they do not depend on the existence of the objects supposedly represented by the formula, but only on the formal manipulation of these formulae. Thus if $Y(T)$ is to exist, then it must be possible to handle the product within $\mathbf{B}$, over all ordered sets of objects, $\left\{A_{0}, \ldots, A_{n}\right\}$, in A. Of course $\oint_{A} T(A, A)$ might exist, even if such products do not exist, but in any case these considerations are extraneous to our work here. Our results describe how $\oint_{A} T(A, A)$ reacts when it exists, regardless of questions related to the size of $\mathbf{A}$. 


\section{Coherent ends and Kan COMPlexes}

In the category of simplicial sets, $S$, the enriched hom-object, $\mathbf{S}(X, Y)$ is a Kan complex if $Y$ is Kan, or, adopting the terminology of Quillen's model category theory, if $Y$ is fibrant. Thus to some extent locally Kan $S$-categories are like categories in which every object is fibrant. This, of course, greatly enriches the potential of the theory as will be seen later. Partially because of this, it is useful to know conditions that imply that $\oint_{A} T(A, A)$ will be a Kan complex for $T$ : $\mathbf{A}^{o p} \times \mathbf{A} \rightarrow \mathbf{S}$. The methods we will use are based heavily on those developed for use with homotopy limits by Bousfield and Kan, [11].

Proposition 2.1. If $\boldsymbol{A}$ is an $S$-category and $T: \mathbf{A}^{o p} \times \mathbf{A} \rightarrow \mathbf{S}$ is an $S$-functor such that each $T\left(A^{\prime}, A^{\prime \prime}\right)$ is a Kan complex, then $\oint_{A} T(A, A)$ is a Kan complex.

Proof. Recall [11], Ch.X, that if $X$ is a cosimplicial simplicial set and $n \geq-1$, the matching space $M^{n} X$ is the simplicial set with simplices $(n+1)$-tuples

$$
\left(x^{0}, \ldots, x^{n}\right) \in X^{n} \times \ldots \times X^{n}
$$

for which $s^{i} x^{j}=s^{j-1} x^{i}$ whenever $0 \leq i<j \leq n$. (Thus $M^{n} X$ is a cosimplicial analogue of the simplicial kernel construction often considered in simplicial set theory.) There is a natural map

$$
s: X^{n+1} \rightarrow M^{n} X
$$

in $S$, given by $s(x)=\left(s^{0} x, \ldots, s^{n} x\right)$ for all $x \in X^{n+1}$. Clearly $M^{-1} X \cong \Delta[0]$ and $M^{0} X=X^{0}$. Following Bousfield and Kan [11], we will say $X$ is fibrant if for each $n \geq-1$

$$
s: X^{n+1} \rightarrow M^{n} X
$$

is a Kan fibration.

A special case of Axiom SM7 on page 277 of [11] (corresponding to $A=\emptyset, B=$ $\Delta, X=X, Y=\Delta[0])$ gives:

If $X$ is a fibrant cosimplicial simplicial set, then Tot $X$ is a Kan complex.

Thus to prove the above proposition it suffices to prove that each

$$
s: Y(T)^{n+1} \rightarrow M^{n} Y(T)
$$

is a fibration. The proof of this is based on the following idea: $s$ is a map between products. By introducing some "dummy" trivial factors into $M^{n} Y(T)$ in a natural way, we can decompose $s$ as a product of simpler maps and it will be sufficient to prove that each of these simpler factors is a Kan fibration. To do this, we will show that each is of the form

$$
\mathbf{S}(X, Y) \rightarrow \mathbf{S}(A, Y)
$$

for $i: A \rightarrow X$ an inclusion/cofibration and $Y$ Kan. The simplicial set $Y(T)^{n}$ in this case has a simpler description

$$
\prod \mathbf{S}\left(\mathbf{A}\left(A_{0}, A_{1}\right) \times \ldots \times \mathbf{A}\left(A_{n-1}, A_{n}\right), T\left(A_{0}, A_{n}\right)\right)
$$

since we are in the base $\mathbf{B}=\mathbf{S}$. The codegeneracies

$$
s^{i}: Y(T)^{n+1} \rightarrow Y(T)^{n}
$$

are induced by the $k_{i}$ 's introduced earlier, given by inclusion of the $i^{\text {th }}$ identity

$$
\Delta[0] \rightarrow \mathbf{A}\left(A_{i}, A_{i}\right) .
$$


We will consider $k_{i}$ as an inclusion of

$$
\mathbf{A}\left(A_{0}, A_{1}\right) \times \ldots \times \mathbf{A}\left(A_{i-1}, A_{i}\right) \times \Delta[0] \times \mathbf{A}\left(A_{i}, A_{i+1}\right) \times \ldots \times \mathbf{A}\left(A_{n-1}, A_{n}\right)
$$

into

$$
\mathbf{A}\left(A_{0}, A_{1}\right) \times \ldots \times \mathbf{A}\left(A_{i-1}, A_{i}\right) \times \mathbf{A}\left(A_{i}, A_{i}\right) \times \mathbf{A}\left(A_{i}, A_{i+1}\right) \times \ldots \times \mathbf{A}\left(A_{n-1}, A_{n}\right) .
$$

For a given indexing sequence $\left(A_{0}, \ldots, A_{n+1}\right)$, we set

$$
\begin{aligned}
& D\left(A_{0}, \ldots, A_{n+1}\right) \\
& \quad=\bigcup_{1 \leq i \leq n+1} \text { Domain of } k_{i}=\left\{\left(a_{0}, \ldots, a_{n}\right) \mid \text { there is some } i\right. \\
& \quad \subseteq \mathbf{A}\left(A_{0}, A_{1}\right) \times \ldots \times \mathbf{A}\left(A_{n}, A_{n+1}\right) .
\end{aligned}
$$

We note that if for each $j, A_{j} \neq A_{j+1}$, then $D\left(A_{0}, \ldots, A_{n+1}\right)$ is empty, since no $k_{i}$ can have codomain $\mathbf{A}\left(A_{0}, A_{1}\right) \times \ldots \times \mathbf{A}\left(A_{n-1}, A_{n}\right)$. Similarly if a repetition $A_{i}=A_{i+1}$ occurs exactly once in the sequence then $D\left(A_{0}, \ldots, A_{n+1}\right)=$ domain of $k_{i}$. With this notation $k_{i}$ can be considered as a composite:

$$
\text { Domain of } k_{i} \rightarrow D\left(A_{0}, \ldots A_{n+1}\right) \rightarrow \mathbf{A}\left(A_{0}, A_{1}\right) \times \ldots \times \mathbf{A}\left(A_{n}, A_{n+1}\right) .
$$

Moreover if $A_{i}=A_{i+1}$ and $A_{j}=A_{j+1}$, Domain of $k_{i} \cap$ Domain of $k_{j}$ can be identified with the simplicial set corresponding to the index

$$
\left(A_{0}, \ldots, A_{i-1}, A_{i}, A_{i+2}, \ldots, A_{j-1}, A_{j}, A_{j+2}, \ldots, A_{n+1}\right) .
$$

Now suppose $f \in \mathbf{S}\left(D\left(A_{0}, \ldots, A_{n+1}\right), T\left(A_{0}, A_{n+1}\right)\right)$. Set $f_{i}=f \mid$ Domain of $k_{i}$ and, abusing notation, write $k_{j}$ for $k_{j} \mid$ (Domain of $k_{i} \cap$ Domain of $k_{j}$ ). Then for $0 \leq i<j \leq n$,

$$
f_{j} k_{i}=f_{i} k_{j}
$$

Similarly if $\left(f_{0}, \ldots, f_{n}\right)$ is a sequence of simplices satisfying the above equations (possibly some with empty domain), then they glue together to give a simplex

$$
f \in \mathbf{S}\left(D\left(A_{0}, \ldots, A_{n+1}\right), T\left(A_{0}, A_{n+1}\right)\right) .
$$

This correspondence between compatible sequences of simplices

$$
\left.f_{i} \in \mathbf{S} \text { (Domain of } k_{i}, T\left(A_{0}, A_{n+1}\right)\right)
$$

and simplices

$$
f \in \mathbf{S}\left(D\left(A_{0}, \ldots, A_{n+1}\right), T\left(A_{0}, A_{n+1}\right)\right)
$$

enables us to

1. write $M^{n} Y(T)$ as a product

$$
\prod \mathbf{S}\left(D\left(A_{0}, \ldots, A_{n+1}\right), T\left(A_{0}, A_{n+1}\right)\right)
$$

with the product taken over all sequences $\left(A_{0}, \ldots, A_{n+1}\right)$ of objects in $\mathbf{A}$, (remember if $\left(A_{0}, \ldots, A_{n+1}\right)$ contains no repetitions, $D\left(A_{0}, \ldots, A_{n+1}\right)$ is empty and

$$
\left.\mathbf{S}\left(D\left(A_{0}, \ldots, A_{n+1}\right), T\left(A_{0}, A_{n+1}\right)\right) \cong \Delta[0]\right)
$$


2. write $s: Y(T)^{n+1} \rightarrow M^{n} Y(T)$ as a product of maps from

$$
\mathbf{S}\left(\mathbf{A}\left(A_{0}, A_{1}\right) \times \ldots \times \mathbf{A}\left(A_{n}, A_{n+1}\right), T\left(A_{0}, A_{n+1}\right)\right)
$$

to

$$
\mathbf{S}\left(D\left(A_{0}, \ldots, A_{n+1}\right), T\left(A_{0}, A_{n+1}\right)\right),
$$

each induced by the inclusion of the corresponding $D\left(A_{0}, \ldots, A_{n+1}\right)$.

As each inclusion

$$
D\left(A_{0}, \ldots, A_{n+1}\right) \rightarrow \mathbf{A}\left(A_{0}, A_{1}\right) \times \ldots \times \mathbf{A}\left(A_{n}, A_{n+1}\right),
$$

is a cofibration and each $T\left(A_{0}, A_{n+1}\right)$ is a Kan complex, each of these factor maps is a Kan fibration, hence so is $s$ itself and the proof is complete.

Corollary 2.2. If $T_{0}, T_{1}: \mathbf{A}^{o p} \times \mathbf{A} \rightarrow \mathbf{S}$ are $S$-functors such that each $T_{0}\left(A^{\prime}, A^{\prime \prime}\right)$ and each $T_{1}\left(A^{\prime}, A^{\prime \prime}\right)$ is a Kan complex and

$$
\left\{\eta\left(A^{\prime}, A^{\prime \prime}\right): T_{0}\left(A^{\prime}, A^{\prime \prime}\right) \rightarrow T_{1}\left(A^{\prime}, A^{\prime \prime}\right)\right\}
$$

is an $S$-natural transformation of $S$-functors such that each $\eta\left(A^{\prime}, A^{\prime \prime}\right)$ is a homotopy equivalence, then $\eta$ induces a homotopy equivalence

$$
\oint_{A} \eta(A, A): \oint_{A} T_{0}(A, A) \rightarrow \oint_{A} T_{1}(A, A) .
$$

Proof. It is immediate that $\eta$ induces a map of cosimplicial simplicial sets $Y(\eta)$ : $Y\left(T_{0}\right) \rightarrow Y\left(T_{1}\right)$ and that $Y(\eta)$ is a weak equivalence in the sense of Bousfield and Kan ([11], Ch.X §4), i.e. that for each $n \geq 0, Y(\eta)^{n}: Y\left(T_{0}\right)^{n} \rightarrow Y\left(T_{1}\right)^{n}$ is a homotopy equivalence.

(In fact they only require that each $Y(\eta)^{n}$ be a weak equivalence, but as $Y\left(T_{0}\right)^{n}$ and $Y\left(T_{1}\right)^{n}$ are Kan complexes, this implies that each $Y(\eta)^{n}$ is a homotopy equivalence.) As a consequence we can conclude, [11], Ch.X $\S 5$, that $\operatorname{Tot} Y(\eta)$ is a homotopy equivalence in $\mathbf{S}$, but Tot $Y(\eta)$ is, of course, $\oint_{A} \eta(A, A)$.

In the next corollary, we extend the proposition above to an arbitrary complete $S$-category $\mathbf{B}$. We will say an object $B$ in $\mathbf{B}$ is fibrant if $\mathbf{B}(X, B)$ is a Kan complex for all objects $X$ in $\mathbf{B}$. Thus if $\mathbf{B}$ is locally Kan, all objects are fibrant. The method we use below will be used many times later in this article.

Corollary 2.3. Let $T: \mathbf{A}^{o p} \times \mathbf{A} \rightarrow \mathbf{B}$ be an $S$-functor where $\mathbf{B}$ is a complete $S$ category and for each $A^{\prime}, A^{\prime \prime}$ in $\mathbf{A}, T\left(A^{\prime}, A^{\prime \prime}\right)$ is fibrant. Then $\oint_{A} T(A, A)$ is fibrant.

Proof. Let $X$ be an arbitrary object of B. One easily checks

$$
\mathbf{B}\left(X, \oint_{A} T(A, A)\right) \cong \oint_{A} \mathbf{B}(X, T(A, A))
$$

since $\mathbf{B}\left(X,{ }_{-}\right)$preserves limits, but as $\mathbf{B}\left(X, T\left({ }_{-},{ }_{-}\right)\right): \mathbf{A}^{o p} \times \mathbf{A} \rightarrow \mathbf{S}$ takes "Kan" values, $\oint_{A} \mathbf{B}(X, T(A, A))$ is Kan. As $X$ was arbitrary, this interprets as saying $\oint_{A} T(A, A)$ is fibrant.

Remark. This corollary is the result mentioned in [21] for extending that version of Vogt's theorem to the case where B is a locally Kan full sub $S$-category of a complete $S$-category and the rectification used in the proof (which was an example of a coherent end) involves fibrant objects, since $\mathbf{B}$ is locally Kan. This use of subcategories of fibrant objects mirrors that given in Quillen's theory, the idea being that on fibrant (cofibrant) objects, the homotopy theory is more easy to 
manipulate. As we are using ends, i.e. limits, here the key ideas relate to fibrations and fibrant objects as in K. S. Brown's, [14], rather than cofibrations and cofibrant objects.

The internal homotopy theory in a locally Kan $S$-category has been mentioned earlier and we will not develop it as such here; however we will define $f: B \rightarrow B^{\prime}$ in an $S$-category $\mathbf{B}$ to be a homotopy equivalence if for all $X$ in $\mathbf{B}, \mathbf{B}(X, f)$ is a homotopy equivalence. This external homotopy equivalence can be internalised provided B is locally Kan and is a full sub $S$-category of a complete (or cocomplete) $S$-category.

Corollary 2.4. If $T_{0}, T_{1}: \mathbf{A}^{o p} \times \mathbf{A} \rightarrow \mathbf{B}, \eta: T_{0} \rightarrow T_{1}$ is a natural homotopy equivalence, and $T_{0}, T_{1}$ take fibrant values, then $\oint_{A} \eta$ is a homotopy equivalence in B.

The proof should now be clear as it combines the methods of 2.2 and 2.3 .

\section{Coherent transformations and the Coherent Yoneda lemma}

Our guiding principle is that when looking for the coherent analogue of categorical constructions, the way to proceed is to replace sets by simplicial sets, categories by $S$-categories, functors by $S$-functors, etc., and ends by coherent ends. It is routine that if $A$ and $B$ are categories and $F, G: A \rightarrow B$ are functors, the set of natural transformations from $F$ to $G$ is given by $\int_{A} B(F A, G A)$. (It is well known, but less routine, that in the analogous 2-categorical situation, one can use lax ends to get the lax analogue.) This suggests the following:

Definition 3.1. Let $F, G: \mathbf{A} \rightarrow \mathbf{B}$ be two $S$-functors; then the simplicial set of coherent transformations from $F$ to $G$ denoted $\operatorname{Coh}(\mathbf{A}, \mathbf{B})(F, G)$ is defined to be

$$
\operatorname{Coh}(\mathbf{A}, \mathbf{B})(F, G)=\oint_{A} \mathbf{B}(F A, G A)
$$

This is thus given as a total object by the formula

$$
\int_{n} \mathbf{S}\left(\Delta[n], \prod_{A_{0}, \ldots, A_{n}} \mathbf{S}\left(\mathbf{A}\left(A_{0}, A_{1}\right) \times \ldots \times \mathbf{A}\left(A_{n-1}, A_{n}\right), \mathbf{B}\left(F A_{0}, G A_{n}\right)\right)\right) .
$$

Example. Let $\mathbf{A}=A$ be an ordinary category, $F, G: A \rightarrow$ Top be two ordinary functors; then

$$
\operatorname{Coh}(A, \mathbf{T o p})(F, G)=\int_{n} \operatorname{Top}\left(\Delta^{n} \times \coprod_{A_{0} \stackrel{f_{1}}{\rightarrow} \ldots \stackrel{f_{n}}{\rightarrow} A_{n}} F A_{0}, G A_{n}\right) .
$$

Specifying a coherent morphism

$$
m: F \rightarrow G
$$

thus corresponds to a 0 -simplex of $\operatorname{Coh}(A, \mathbf{T o p})(F, G)$, that is, to the specification of a higher 'homotopy',

$$
h\left(f_{1}, \ldots, f_{n}\right): F A_{0} \times \Delta^{n} \rightarrow G A_{n},
$$

for each $n$-tuple of composable morphisms, $A_{0} \stackrel{f_{1}}{\rightarrow} A_{1} \rightarrow \ldots \stackrel{f_{n}}{\rightarrow} A_{n}$. These homotopies are compatible with each other in the following sense (essentially read off 
from the face and degeneracy information encoded in the coherent end):

$$
\begin{aligned}
& h\left(f_{1}, \ldots, f_{n}\right)\left(x, v_{1} \leq \ldots \leq v_{n}\right) \text { is } h\left(f_{2}, \ldots, f_{n}\right)\left(F\left(f_{1}\right) x, v_{2} \leq \ldots \leq v_{n}\right) \text { if } v_{1}=0 ; \\
& h\left(f_{1}, \ldots, f_{i+1} f_{i}, \ldots, f_{n}\right)\left(x, v_{1} \leq \ldots \leq v_{i} \leq v_{i+2} \leq \ldots \leq v_{n}\right) \\
& \text { if } v_{i}=v_{i+1} \text { for } 0<i<n ; \\
& G\left(f_{n}\right) h\left(f_{1}, \ldots, f_{n-1}\right)\left(x, v_{1} \leq \ldots \leq v_{n-1}\right) \text { if } v_{n}=1 ; \text { and } \\
& h\left(f_{1}, \ldots, f_{i-1}, f_{i+1}, \ldots, f_{n}\right)\left(x, v_{1} \leq \ldots \leq v_{i-1} \leq v_{i+1} \leq \ldots \leq v_{n}\right) \text { if } f_{i}=i d .
\end{aligned}
$$

Remark. 1 . These are only the 0 -simplices. There are higher order simplices giving homotopies between these coherent morphisms and so on.

2. It is simple to extend this detailed description to the case where $F, G$ are homotopy coherent diagrams of type $A$. One replaces $A$ by $\mathbf{S}(A)$, the $S$-category resolving $A$, cf. Cordier, [16], and also [21]. The data for a coherent transformation as defined geometrically in those papers is slightly more general than that used here. The two simplicial sets have the same homotopy type however since the "receiving category" Top is locally Kan.

Proposition 3.2. Given $F, G: \mathbf{A} \rightarrow \mathbf{B}$, where $\mathbf{B}$ is locally Kan, then the simplicial set $\operatorname{Coh}(\mathbf{A}, \mathbf{B})(F, G)$ is a Kan complex.

The proof is immediate from the definition given the results of section 2. Later on we will examine the question of composition of coherent transformations, but before that we will look at a representation of coherent transformations as being $S$-natural transformations between related $S$-functors.

The augmentation of the indexation. A technical tool we shall use several times in the following is the augmentation of the indexation functor.

The bisimplicial set $X(A, B)$ comes with a natural augmentation

$$
d_{0}: X(A, B)_{0} \rightarrow \mathbf{A}(A, B)
$$

given by composition:

$$
X(A, B)_{0}=\coprod_{A_{0}} \mathbf{A}\left(A, A_{0}\right) \times \mathbf{A}\left(A_{0}, B\right) \rightarrow \mathbf{A}(A, B) .
$$

This has a homotopy inverse given by $s_{-1}$, i.e. it sends $g \in \mathbf{A}(A, B)_{n}$ to $\left(i d_{A}, g\right)$. These two maps pass to the diagonal to give

$$
d_{0}: \hat{\mathbf{A}}(A, B) \rightarrow \mathbf{A}(A, B),
$$

and

$$
s_{-1}: \mathbf{A}(A, B) \rightarrow \hat{\mathbf{A}}(A, B),
$$

which are both homotopy equivalences. The important difference between them is that whilst $d_{0}$ is natural in $A$ and $B, s_{-1}$ is natural in $B$ but not in $A$. Furthermore whilst $d_{0} s_{-1}$ is the identity, $s_{-1} d_{0}$ is homotopic to the identity by a homotopy which, again, is natural in $B$ but not in $A$. In fact both $s_{-1}$ and this homotopy are coherent in $A$ as is easily checked.

Now suppose $\mathbf{B}$ is a complete locally Kan $S$-category and that $F: \mathbf{A} \rightarrow \mathbf{B}$, $G: \mathbf{A} \rightarrow \mathbf{S}$ are $S$-functors. We define the coherent mean cotensor of $F$ and $G$ by

$$
\overline{B^{A}}(G, F)=\oint_{A} \overline{\mathbf{B}}(G A, F A) .
$$


In particular, we get a new functor $\bar{F}: \mathbf{A} \rightarrow \mathbf{B}$, which is defined by $\bar{F}(A)=$ $\overline{B^{A}}\left(\mathbf{A}\left(A,{ }_{-}\right), F(-)\right)$.

For $S$-functors, $F, G: \mathbf{A} \rightarrow \mathbf{B}, \mathbf{B}^{\mathbf{A}}(G, F)=\int_{A} \mathbf{B}(G A, F A)$ is the simplicial set of natural transformations, so this coherent mean cotensor generalises this both by replacing $G$ by $G: \mathbf{A} \rightarrow \mathbf{S}$ and by replacing the end by a coherent end.

Dually if $G: \mathbf{A}^{o p} \rightarrow \mathbf{S}$, then the coherent mean tensor, $G \bar{\otimes} F$, will exist if $\mathbf{B}$ is cocomplete:

$$
G \bar{\otimes} F=\oint^{A} G A \bar{\otimes} F A .
$$

In particular we will write $\underline{F}: \mathbf{A} \rightarrow \mathbf{B}$ for the functor given by $\underline{F}(A)=$ $\mathbf{A}\left({ }_{-}, A\right) \bar{\otimes} F$.

These functors $\bar{F}$ and $\underline{F}$ "absorb coherence" as follows:

Proposition 3.3. (i) If $\mathbf{B}$ is complete, there is a natural isomorphism

$$
\operatorname{Coh}(\mathbf{A}, \mathbf{B})(F, G) \cong \mathbf{B}^{A}(F, \bar{G})
$$

for any $F, G: \mathbf{A} \rightarrow \mathbf{B}$, where, as above, $\mathbf{B}^{\mathbf{A}}(F, \bar{G})$ is the simplicial set of natural transformations from $F$ to $\bar{G}$.

(ii) If $\mathbf{B}$ is cocomplete, there is a natural isomorphism

$$
\operatorname{Coh}(\mathbf{A}, \mathbf{B})(F, G) \cong \mathbf{B}^{A}(\underline{F}, G) .
$$

Proof. We will only give the proof of (ii). That of (i) is dual.

$$
\begin{aligned}
\mathbf{B}^{A}(\underline{F}, G) & =\int_{A} \mathbf{B}(\underline{F} A, G A) \\
& =\int_{A} \mathbf{B}\left(\int^{A^{\prime}, A^{\prime \prime}}\left(\widehat{\mathbf{A}^{o p}}\right)\left(A^{\prime}, A^{\prime \prime}\right) \bar{\otimes}\left(\mathbf{A}\left(A^{\prime}, A\right) \bar{\otimes} F A^{\prime \prime}\right), G A\right) \\
& \cong \int_{A, A^{\prime}, A^{\prime \prime}} \mathbf{S}\left(\operatorname{Diag} X\left(A^{\prime \prime}, A^{\prime}\right) \times \mathbf{A}\left(A^{\prime}, A\right), \mathbf{B}\left(F A^{\prime \prime}, G A\right)\right) \\
& \cong \int_{A, A^{\prime \prime}} \mathbf{S}\left(\hat{\mathbf{A}}\left(A^{\prime \prime}, A\right), \mathbf{B}\left(F A^{\prime \prime}, G A\right)\right) \\
& =\operatorname{Coh}(\mathbf{A}, \mathbf{B})(F, G) .
\end{aligned}
$$

Examples. 1. For fixed $A$ in $\mathbf{A}$, let $F=\mathbf{A}\left(A,_{-}\right): \mathbf{A} \rightarrow \mathbf{S}$, then

$$
\begin{aligned}
\underline{F}(B) & =\int^{A^{\prime}, A^{\prime \prime}}\left(\widehat{\mathbf{A}^{o p}}\right)\left(A^{\prime}, A^{\prime \prime}\right) \times \mathbf{A}\left(A^{\prime}, B\right) \times \mathbf{A}\left(A, A^{\prime \prime}\right) \\
& \cong \int^{A^{\prime}, A^{\prime \prime}} \mathbf{A}\left(A, A^{\prime \prime}\right) \times \hat{\mathbf{A}}\left(A^{\prime \prime}, A^{\prime}\right) \times \mathbf{A}\left(A^{\prime}, B\right) \\
& \cong \hat{\mathbf{A}}(A, B) .
\end{aligned}
$$

The isomorphism of 3.3 (ii) specialises to give

$$
\mathbf{S}^{A}\left(\hat{\mathbf{A}}\left(A,_{-}\right), \mathbf{A}\left(A,,_{-}\right)\right) \cong C o h(\mathbf{A}, \mathbf{S})\left(\mathbf{A}\left(A,_{-}\right), \mathbf{A}\left(A,_{-}\right)\right)
$$

and the $S$-natural transformation corresponding to the identity coherent transformation is $d_{0}$, as it is induced by composition.

2. Reversing the rôles of $\hat{\mathbf{A}}\left(A,,_{-}\right)$and $\mathbf{A}\left(A,,_{-}\right)$, we get that to the identity in

$$
\mathbf{S}^{A}\left(\hat{\mathbf{A}}\left(A,_{-}\right), \hat{\mathbf{A}}\left(A,_{-}\right)\right)
$$


there corresponds a coherent transformation, $\sigma$, in

$$
\operatorname{Coh}(\mathbf{A}, \mathbf{S})\left(\mathbf{A}\left(A,{ }_{-}\right), \hat{\mathbf{A}}\left(A,{ }_{-}\right)\right)_{0} .
$$

Direct calculation of $\sigma$ shows it to be given by a natural map

$$
\sigma: \hat{\mathbf{A}}\left(A^{\prime \prime},{ }_{-}\right) \rightarrow \mathbf{S}\left(\mathbf{A}\left(A, A^{\prime \prime}\right), \hat{\mathbf{A}}\left(A,_{-}\right)\right)
$$

which is adjoint to the $\mathbf{A}$-action

$$
\bar{\sigma}: \mathbf{A}\left(A, A^{\prime \prime}\right) \times \hat{\mathbf{A}}\left(A^{\prime \prime},{ }_{-}\right) \rightarrow \hat{\mathbf{A}}\left(A,_{-}\right)
$$

given by composition. Thus to the identity string in $\hat{\mathbf{A}}\left(A^{\prime \prime}, A^{\prime \prime}\right)$, there corresponds a natural map $\sigma(i d): \mathbf{A}\left(A, A^{\prime \prime}\right) \rightarrow \hat{\mathbf{A}}\left(A, A^{\prime \prime}\right)$ and if $g \in \mathbf{A}\left(A, A^{\prime \prime}\right)_{n}$, then $\sigma(i d)(g)=$ $\left(i d_{A}, g\right)$. In other words $\sigma(i d)$ is the homotopy inverse to the augmentation and is, in fact, a natural transformation in the variable $A^{\prime \prime}$.

3. In general if $\mathbf{B}$ is complete and $F: \mathbf{A} \rightarrow \mathbf{B}$, then there is a natural transformation,

$$
\eta_{F}: F \rightarrow \bar{F}
$$

corresponding, via 3.2 (i), to the identity coherent transformation on $F$. Dually if $\mathbf{B}$ is cocomplete, there is a natural transformation

$$
\eta^{F}: \underline{F} \rightarrow F
$$

Proposition 3.4. (i) If $\mathbf{B}$ is complete, then $\eta_{F}: F \rightarrow \bar{F}$ is a levelwise homotopy equivalence, i.e. for each $A$ in $\mathbf{A}, \eta_{F}(A)$ is a homotopy equivalence.

(ii) If $\mathbf{B}$ is cocomplete then $\eta^{F}: \underline{F} \rightarrow F$ is a levelwise homotopy equivalence.

Before we prove this, we extend and adapt some ideas and results from CordierPorter, [21].

Suppose $F, F^{\prime}: \mathbf{A} \rightarrow \mathbf{S}, G: \mathbf{A} \rightarrow \mathbf{B}$ are $S$-functors, $f_{0}, f_{1}: F \rightarrow F^{\prime}$ are two natural transformations and $h: F \times \Delta[1] \rightarrow F^{\prime}$ is a natural homotopy between them. Suppose that $\mathbf{B}$ is cotensored; then we can form $\int_{A} \overline{\mathbf{B}}(F A, G A)$ and $\int_{A} \overline{\mathbf{B}}\left(F^{\prime} A, G A\right)$ and $f_{0}, f_{1}$ induce natural transformations

$$
f_{0}^{\#}, f_{1}^{\#}: \int_{A} \overline{\mathbf{B}}\left(F^{\prime} A, G A\right) \rightarrow \int_{A} \overline{\mathbf{B}}(F A, G A) .
$$

Proposition 3.5. The natural homotopy $h: f_{0} \simeq f_{1}$ induces a natural homotopy $h^{\#}: f_{0}^{\#} \simeq f_{1}^{\#}$.

Proof. The natural transformation $h$ induces a map

$$
\int_{A} \overline{\mathbf{B}}(h A, G A): \int_{A} \overline{\mathbf{B}}\left(F^{\prime} A, G A\right) \rightarrow \int_{A} \overline{\mathbf{B}}(F A \times \Delta[1], G A)
$$

(which we will normally shorten to $\int_{A} h$ ), but

$$
\begin{aligned}
\int_{A} \overline{\mathbf{B}}(F A \times \Delta[1], G A) & \cong \int_{A} \overline{\mathbf{B}}(\Delta[1], \overline{\mathbf{B}}(F A, G A)) \\
& \cong \overline{\mathbf{B}}\left(\Delta[1], \int_{A} \overline{\mathbf{B}}(F A, G A)\right)
\end{aligned}
$$

and it is easily checked that $h^{\#}=\int_{A} h$ gives the required homotopy. 
Corollary 3.6. Suppose $F, F^{\prime}: \mathbf{A} \rightarrow \mathbf{S}$. If $f: F \rightarrow F^{\prime}$ and $g: F^{\prime} \rightarrow F$ are natural homotopy inverses (i.e. there are natural homotopies $h: f g \simeq I d, k: g f \simeq I d$ ) then for any $G: \mathbf{A} \rightarrow \mathbf{B}$ with $\mathbf{B}$ cocomplete, $\int_{A} f$ and $\int_{A} g$ form a homotopy equivalence between $\int_{A} \overline{\mathbf{B}}(F A, G A)$ and $\int_{A} \overline{\mathbf{B}}\left(F^{\prime} A, G A\right)$.

The proof is a simple application of 3.4 .

There are, of course, dual versions of 3.5 and 3.6 whose formulation and proof we leave to the reader.

Proof of 3.4 (i). Since by the Yoneda lemma

$$
F A=\int_{A^{\prime}} \overline{\mathbf{B}}\left(\mathbf{A}\left(A, A^{\prime}\right), F A^{\prime}\right)
$$

whilst

$$
\bar{F} A \cong \int_{A^{\prime}} \overline{\mathbf{B}}\left(\hat{\mathbf{A}}\left(A, A^{\prime}\right), F A^{\prime}\right),
$$

the natural homotopy inverses $d_{0}: \hat{\mathbf{A}}\left(A,_{-}\right) \rightarrow \mathbf{A}\left(A,_{-}\right)$and $s_{-1}: \mathbf{A}\left(A,_{-}\right) \rightarrow \hat{\mathbf{A}}\left(A,_{-}\right)$ provide the solution to constructing a homotopy equivalence from $F A$ to $\bar{F} A$. It remains to check that this is $\eta_{F}$, but this is routine, using the usual techniques of the proof of the Yoneda lemma.

The proof of 3.4 (ii) is dual, using the dual forms of 3.5 and 3.6. It is important to note that although $\eta_{F}$ is a natural (levelwise) homotopy equivalence, its homotopy inverse $\int_{A} s_{-1}$ is not natural.

Corollary 3.7. Suppose that $\mathbf{B}$ is a complete cotensored locally Kan S-category and $F, G: \mathbf{A} \rightarrow \mathbf{B}$ two $S$-functors. Then the augmentation induces a homotopy equivalence

$$
\operatorname{Coh}(\mathbf{A}, \mathbf{B})(G, F) \rightarrow \operatorname{Coh}(\mathbf{A}, \mathbf{B})(G, \bar{F})
$$

Remark. Noting that $\operatorname{Coh}(\mathbf{A}, \mathbf{B})(G, F) \cong \mathbf{B}^{A}(G, \bar{F})$ by $3.2(\mathrm{i})$, this homotopy equivalence is the natural inclusion (up to identification via this isomorphism) of $\mathbf{B}^{A}(G, \bar{F})$ into $\operatorname{Coh}(\mathbf{A}, \mathbf{B})(G, \bar{F})$.

Proof of 3.7. First we write $\bar{F} A \cong \int_{A^{\prime}} \overline{\mathbf{B}}\left(\hat{\mathbf{A}}\left(A, A^{\prime}\right), F A^{\prime}\right)$ by the isomorphism used previously; then

$$
\begin{aligned}
C o h(\mathbf{A}, \mathbf{B})(G, \bar{F}) & \cong \int_{A, A^{\prime}} \mathbf{S}\left(\hat{\mathbf{A}}(A, A), \mathbf{B}\left(G A, \int_{A^{\prime \prime}} \overline{\mathbf{B}}\left(\hat{\mathbf{A}}\left(A^{\prime}, A^{\prime \prime}\right), F A^{\prime \prime}\right)\right)\right) \\
& \cong \int_{A, A^{\prime}} \mathbf{S}\left(\hat{\mathbf{A}}\left(A, A^{\prime}\right), \int_{A^{\prime \prime}} \mathbf{S}\left(\hat{\mathbf{A}}\left(A^{\prime}, A^{\prime \prime}\right), \mathbf{B}\left(G A, F A^{\prime \prime}\right)\right)\right) .
\end{aligned}
$$

Similarly

$$
C o h(\mathbf{A}, \mathbf{B})(G, F) \cong \int_{A, A^{\prime}} \mathbf{S}\left(\hat{\mathbf{A}}\left(A, A^{\prime}\right), \int_{A^{\prime \prime}} \mathbf{S}\left(\mathbf{A}\left(A^{\prime}, A^{\prime \prime}\right), \mathbf{B}\left(G A, F A^{\prime \prime}\right)\right)\right) .
$$

Writing

$$
H\left(A, A^{\prime}\right)=\int_{A^{\prime \prime}} \mathbf{S}\left(\hat{\mathbf{A}}\left(A^{\prime}, A^{\prime \prime}\right), \mathbf{B}\left(G A, F A^{\prime \prime}\right)\right)
$$

and

$$
K(A, A)=\int_{A^{\prime \prime}} \mathbf{S}\left(\mathbf{A}\left(A^{\prime}, A^{\prime \prime}\right), \mathbf{B}\left(G A, F A^{\prime \prime}\right)\right),
$$


then

a. $H, K: \mathbf{A}^{o p} \times \mathbf{A} \rightarrow \mathbf{S}$ take Kan values since $\mathbf{B}$ is locally Kan,

b. the augmentation $d_{0}: \hat{\mathbf{A}}\left(A^{\prime}, A^{\prime \prime}\right) \rightarrow \mathbf{A}\left(A^{\prime}, A^{\prime \prime}\right)$ induces, by 3.6 , a homotopy equivalence

$$
K\left(A, A^{\prime}\right) \rightarrow H\left(A, A^{\prime}\right)
$$

natural in $A$ and $A^{\prime}$,

c. $\operatorname{Coh}(\mathbf{A}, \mathbf{B})(G, F) \cong \oint_{A} K(A, A)$ and $\operatorname{Coh}(\mathbf{A}, \mathbf{B})(G, \bar{F}) \cong \oint_{A} H(A, A)$, so the result follows from 2.2.

Remark. Proposition 3.4(i) is a strong form of what might be called the homotopy coherent Yoneda lemma. If $\mathbf{B}=\mathbf{S}$, this then reads as: for each $F: \mathbf{A} \rightarrow \mathbf{S}$, there is a homotopy equivalence

$$
\eta_{F}(A): F(A) \rightarrow \operatorname{Coh}(\mathbf{A}, \mathbf{S})\left(\mathbf{A}\left(A,{ }_{-}\right), F\right) .
$$

In the next section we will need to use the description of $\bar{F} A$ as a total object of a cosimplicial object and to this we turn next. If $F: \mathbf{A} \rightarrow \mathbf{B}$ and $A$ is in $\mathbf{A}$, then

$$
\begin{aligned}
\bar{F} A & =\int_{A^{\prime}, A^{\prime \prime}} \overline{\mathbf{B}}\left(\hat{\mathbf{A}}\left(A^{\prime}, A^{\prime \prime}\right), \overline{\mathbf{B}}\left(\mathbf{A}\left(A, A^{\prime}\right), F A^{\prime \prime}\right)\right) \\
& \cong \int_{A^{\prime}, A^{\prime \prime}} \overline{\mathbf{B}}\left(\mathbf{A}\left(A, A^{\prime}\right) \times \hat{\mathbf{A}}\left(A^{\prime}, A^{\prime \prime}\right), F A^{\prime \prime}\right) \\
& \cong \int_{A^{\prime \prime}} \overline{\mathbf{B}}\left(\hat{\mathbf{A}}\left(A, A^{\prime \prime}\right), F A^{\prime \prime}\right) \\
& =\int_{[n]} \int_{A^{\prime \prime}} \overline{\mathbf{B}}\left(X\left(A, A^{\prime \prime}\right)_{n} \times \Delta[n], F A^{\prime \prime}\right) \\
& \cong \int_{[n]} \overline{\mathbf{B}}\left(\Delta[n], \int_{A^{\prime \prime}} \overline{\mathbf{B}}\left(X\left(A, A^{\prime \prime}\right)_{n}, F A^{\prime \prime}\right)\right),
\end{aligned}
$$

so we take $Y(F, A)_{n}=\int_{A^{\prime \prime}} \overline{\mathbf{B}}\left(X\left(A, A^{\prime \prime}\right), F A^{\prime \prime}\right)$ to get a cosimplicial object in $\mathbf{B}$, whose total object, $\operatorname{hom}(\Delta, Y(F, A))$, is $\bar{F} A$. We leave to the reader the task of writing this in its product form.

Given this description, we note that there is a natural map from $\Delta$ to $*$, the constant cosimplicial simplicial set with value $\Delta[0]$. This induces a map with codomain $\bar{F} A$ from the object defined by the end

$$
\int_{n} \overline{\mathbf{B}}\left(*, \int_{A^{\prime \prime}} \overline{\mathbf{B}}\left(X\left(A, A^{\prime \prime}\right)_{n}, F A^{\prime \prime}\right)\right)
$$

i.e. $\operatorname{hom}(*, Y(F, A))$. The following proposition is sometimes of use.

Proposition 3.8. There is a natural isomorphism

$$
F(A) \cong \operatorname{hom}(*, Y(F, A))
$$

such that, modulo identifying these two objects, the natural map

$$
\operatorname{hom}(*, Y(F, A)) \rightarrow \bar{F}(A)
$$

induced by $\Delta \rightarrow *$ is the map $\eta_{F}(A)$. 
Proof. The proof is simply to note that as $*$ is constant in $n$,

$$
\begin{aligned}
\int_{n} \overline{\mathbf{B}}\left(*, \int_{A^{\prime \prime}} \overline{\mathbf{B}}\left(X\left(A, A^{\prime \prime}\right)_{n}, F A^{\prime \prime}\right)\right) & \cong \overline{\mathbf{B}}\left(*, \int_{A^{\prime \prime}} \overline{\mathbf{B}}\left(\operatorname{colim}_{n} X\left(A, A^{\prime \prime}\right)_{n}, F A^{\prime \prime}\right)\right) \\
& \cong \overline{\mathbf{B}}\left(*, \int_{A^{\prime \prime}} \overline{\mathbf{B}}\left(\mathbf{A}\left(A, A^{\prime \prime}\right), F\left(A^{\prime \prime}\right)\right)\right. \\
& \cong F A
\end{aligned}
$$

by the Yoneda lemma. The identification of the induced map as $\eta_{F}(A)$ is now easy.

Corollary 3.9. Suppose $F, G: \mathbf{A} \rightarrow \mathbf{B}$ are $S$-functors. The natural map from $\Delta$ to $*$ induces a map

$$
\operatorname{hom}(*, Y(F, G)) \rightarrow \operatorname{hom}(\Delta, Y(F, G))
$$

which is isomorphic to the natural inclusion,

$$
\mathbf{B}^{A}(F, G) \rightarrow \operatorname{Coh}(\mathbf{A}, \mathbf{B})(F, G) .
$$

The proof of this using $\eta_{G}: G \rightarrow \bar{G}$ and the previous result is clear. There is also a proof establishing the natural isomorphism

$$
\operatorname{hom}(*, Y(F, G)) \cong \mathbf{B}^{A}(F, G)
$$

directly.

We next turn to the problem of 'interpreting' the simplices in the simplicial set,

$$
\operatorname{Coh}(\mathbf{A}, \mathbf{B})(F, G)
$$

(which we will sometimes abbreviate to $\operatorname{Coh}(F, G)$ if the context is clear). One expects 0-simplices of $\operatorname{Coh}(F, G)$ to correspond to families of maps,

$$
\left\{f_{A}: F(A) \rightarrow G(A)\right\}
$$

indexed by the objects of $\mathbf{A}$, together with higher homotopy information on the "homotopy commutativity" of various "diagrams". We have already briefly seen this earlier when we looked at the geometric example of $\mathbf{B}=T o p$ and $A$, an ordinary category with $F, G$ ordinary functors. Our aim is thus to make precise what it means to say that $\left\{f_{A}\right\}$, as above, is "coherent in $A$ " as one might say " $\left\{f_{A}\right\}$ is natural in $A$ ".

Suppose $f \in \operatorname{Coh}(F, G)_{0}$. Using our interpretation of $\operatorname{Coh}(F, G)$ as the total object of $Y(F, G)$, we have that $f: \Delta \rightarrow Y(F, G)$ and hence $f=\left(f^{n}\right)$ where $f^{n}: \Delta[n] \rightarrow Y(F, G)^{n}$ and $d^{i} f^{n+1}=f^{n} d^{i}$.

We start by examining $n=0$. Thus, referring back to our interpretation of the elements of $\oint_{A} T(A, A)_{0}$ in section $1, f(A)=p(A) f^{0} \in \mathbf{S}(F A, G A)_{0}$, so is a map from $F A$ to $G A$ in $\mathbf{B}$, whilst

$$
p\left(A_{0}, A_{1}\right) f^{1}: \mathbf{A}\left(A_{0}, A_{1}\right) \times \Delta[1] \rightarrow \mathbf{B}\left(F A_{0}, G A_{1}\right) .
$$

For simplicity of interpretation we assume that $\mathbf{B}$ is tensored and hence we can rewrite $p\left(A_{0}, A_{1}\right) f^{1}$ as a homotopy from $\mathbf{A}\left(A_{0}, A_{1}\right) \bar{\otimes} F A_{0}$ to $G A_{1}$. The two ends of this homotopy then correspond to the two composites around the square

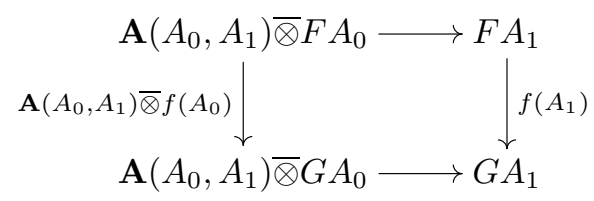


where the horizontal arrows come from the actions of $\mathbf{A}$ on $F$, and $G$, respectively, adjoint to the structure maps, $\mathbf{A}\left(A_{0}, A_{1}\right) \rightarrow \mathbf{B}\left(F A_{0}, F A_{1}\right)$, etc. Similarly in the next dimension, the actions

$$
\begin{aligned}
& \left(\mathbf{A}\left(A_{0}, A_{1}\right) \times \mathbf{A}\left(A_{1}, A_{2}\right)\right) \bar{\otimes} F A_{0} \quad \rightarrow \quad F A_{2}, \\
& \left(\mathbf{A}\left(A_{0}, A_{1}\right) \times \mathbf{A}\left(A_{1}, A_{2}\right)\right) \bar{\otimes} F A_{0} \rightarrow \mathbf{A}\left(A_{1}, A_{2}\right) \bar{\otimes} F A_{1}
\end{aligned}
$$

and

$$
\mathbf{A}\left(A_{1}, A_{2}\right) \bar{\otimes} F A_{1} \rightarrow F A_{2},
$$

and similarly for $G$, are linked via the various $f(A) s$ and the higher homotopy

$$
p\left(A_{0}, A_{1}, A_{2}\right) f^{2}:\left(\mathbf{A}\left(A_{0}, A_{1}\right) \times \mathbf{A}\left(A_{1}, A_{2}\right) \times \Delta[2]\right) \bar{\otimes} F A_{0} \rightarrow G A_{2}
$$

provides a homotopy linking them. More accurately the faces of this are:

$$
\begin{array}{lll}
d_{0} \text { is } & \left(\mathbf{A}\left(A_{1}, A_{2}\right) \times \Delta[1]\right) \bar{\otimes} F A_{1} \stackrel{p\left(A_{1}, A_{2}\right) f^{1}}{\longrightarrow} G A_{2}, \\
d_{1} \text { is } \quad\left(\mathbf{A}\left(A_{0}, A_{2}\right) \times \Delta[1]\right) \bar{\otimes} F A_{0} \stackrel{p\left(A_{0}, A_{2}\right) f^{1}}{\longrightarrow} G A_{2}, \\
d_{2} \text { is } \quad\left(\mathbf{A}\left(A_{0}, A_{1}\right) \times \Delta[1]\right) \bar{\otimes} F A_{0} \stackrel{p\left(A_{0}, A_{1}\right) f^{1}}{\longrightarrow} G A_{1} .
\end{array}
$$

Each of these correspond to a square as above and these squares fit to form a homotopy coherent prism. It is however clearest to think of the higher homotopy $f^{2}$ in the original form as

$$
\left(\mathbf{A}\left(A_{0}, A_{1}\right) \times \mathbf{A}\left(A_{1}, A_{2}\right) \times \Delta[2]\right) \bar{\otimes} F A_{0} \rightarrow G A_{2}
$$

(or

$$
\left.\mathbf{A}\left(A_{0}, A_{1}\right) \times \mathbf{A}\left(A_{1}, A_{2}\right) \times \Delta[2] \rightarrow \mathbf{B}\left(F A_{0}, G A_{2}\right)\right),
$$

together with the face information. We leave the reader the joy of writing down the information corresponding to $p\left(A_{0}, A_{1}, A_{2}, A_{3}\right) f^{3}$ etc.

It should now be clear how to specify what it means for a family $\{f(A): F A \rightarrow$ $G A\}$ to be coherent in $A$, namely the existence of homotopies, higher homotopies, etc. linking the $f(A)$ with the iterated actions of $\mathbf{A}$.

\section{ORDINAL SUM AND COMPOSITION OF COHERENT TRANSFORMATIONS}

The description of $\operatorname{Coh}(\mathbf{A}, \mathbf{B})(F, G)$ as $N a t(F, \bar{G})$ is reminiscent of the construction of a Kleisli category (cf. Mac Lane, [38]) where, given a monad $(T, \eta, \mu)$ on a category, $\mathbf{C}, \mathbf{K l}_{T}$ is the category having the same objects as $\mathbf{C}$, but with $\mathbf{K l}_{T}\left(C_{1}, C_{2}\right)=\mathbf{C}\left(C_{1}, T C_{2}\right)$. Composition of these morphisms is given by

$$
\mathbf{C}\left(C_{1}, T C_{2}\right) \times \mathbf{C}\left(C_{2}, T C_{3}\right) \rightarrow \mathbf{C}\left(C_{1}, T C_{2}\right) \times \mathbf{C}\left(T C_{2}, T^{2} C_{3}\right),
$$

followed by composition in $\mathbf{C}$ to get to $\mathbf{C}\left(C_{1}, T^{2} C_{3}\right)$; finally to arrive back where we want to (i.e. in $\mathbf{C}\left(C_{1}, T C_{3}\right)$ ), we use $\mu: T^{2} \rightarrow T$, the multiplication of the monad. By analogy if we have $\mathbf{S}$-functors $F, G, H$ from $\mathbf{A}$ to $\mathbf{B}$, we can define

$$
\operatorname{Coh}(F, G) \times \operatorname{Coh}(G, H) \rightarrow \operatorname{Nat}(F, \bar{G}) \times \operatorname{Nat}(\bar{G}, \overline{\bar{H}}),
$$

and then compose to get to $N a t(F, \overline{\bar{H}})$. The analogy with the Kleisli construction then suggests that we look for a natural map from $\overline{\bar{H}}$ to $\bar{H}$ satisfying properties analogous to the multiplication of a monad, $\mu: T^{2} \rightarrow T$. The first author found such a map for the case where $B$ is Top (cf. [18]). The construction given there involved a subdivision of the topological $n$-simplex for each $n$. This allowed compositions to be made consistently and naturally. This subdivision had previously been used by 
Lisica and Mardešić in their theory of coherent prohomotopy [37] and the connection with monad structures has recently been noted independently by Batanin, [7]. In general $\mu$ does not exist in such an elegant form. The best one can do is to define a $\mu$ up to homotopy. This again involves subdivision in a natural way and links up this area with various important classical results of algebraic topology.

We thus need to look first at $\overline{\bar{H}}$. Suppose $A$ is an object of $\mathbf{A}$; then

$$
\begin{aligned}
\overline{\bar{H}}(A) & =\oint_{A^{\prime}} \overline{\mathbf{B}}\left(\mathbf{A}\left(A, A^{\prime}\right), \oint_{A^{\prime \prime}} \overline{\mathbf{B}}\left(\mathbf{A}\left(A^{\prime}, A^{\prime \prime}\right), H A^{\prime \prime}\right)\right) \\
& \cong \int_{A_{0}, A_{1}, A_{2}} \overline{\mathbf{B}}\left(\hat{\mathbf{A}}\left(A_{0}, A_{1}\right), \overline{\mathbf{B}}\left(\mathbf{A}\left(A, A_{0}\right), \overline{\mathbf{B}}\left(\hat{\mathbf{A}}\left(A_{2}, A_{3}\right), \overline{\mathbf{B}}\left(\mathbf{A}\left(A_{1}, A_{2}\right), H A_{3}\right)\right)\right)\right) \\
& \cong \int_{A_{0}, A_{1}, A_{2}, A_{3}} \overline{\mathbf{B}}\left(\mathbf{A}\left(A, A_{0}\right) \times \hat{\mathbf{A}}\left(A_{0}, A_{1}\right) \times \mathbf{A}\left(A_{1}, A_{2}\right) \times \hat{\mathbf{A}}\left(A_{2}, A_{3}\right), H A_{3}\right) \\
& \cong \int_{[p],[q]} \int_{A_{0}, A_{1}, A_{2}, A_{3}} \overline{\mathbf{B}}\left(\mathbf{A}\left(A, A_{0}\right) \times X\left(A_{0}, A_{1}\right)_{p}\right. \\
& \cong \int_{A_{0}, A_{3}} \overline{\mathbf{B}}\left(\mathbf{A}\left(A, A_{1}, A_{2}\right) \times X\left(A_{2}, A_{3}\right)_{q} \times \Delta[p] \times \Delta[q], H A_{3}\right) \\
& \cong \int_{[n]} \overline{\mathbf{B}}\left(S d \Delta[n], \int_{A_{0}, A_{3}} \overline{\mathbf{B}}\left(X\left(A_{0}\right)_{p+q+1} \times \Delta[p] \times \Delta[q], H A_{3}\right)\right.
\end{aligned}
$$

by using the reverse process and the analogue of the argument in Proposition 1.2 (Cosimplicial Replacement). Here we have used $S d \Delta[n]$ as a shorthand for

$$
\int_{[p],[q]} \Delta([p]+[q],[n]) \times \Delta[p] \times \Delta[q] .
$$

It is this cosimplicial simplicial set that is the "subdivision" of $\Delta$ mentioned above. The ordinal sum, $[p]+[q]$, of $[p]$ and $[q]$ will be looked at in detail later.

We thus have a natural map from $\overline{\bar{H}}(A)$ to $\operatorname{hom}(S d \Delta, Y(H, A))$, where $Y(H, A)$ satisfies $\bar{H}(A)=\operatorname{hom}(\Delta, Y(H, A))$. The argument if $\mathbf{B}$ is Top (given by the first author in [18]) is essentially to replace $S d \Delta$ by $|S d \Delta|$ and to note that each $|S d \Delta[n]|$ is homeomorphic to the corresponding $|\Delta[n]|$, i.e. to the corresponding $\Delta^{n}$, thus showing that $\bar{H}(A) \cong \operatorname{hom}(S d \Delta, Y(H, A))$. If $\mathbf{B}$ is the $S$-category of simplicial abelian groups, again

$$
\bar{H}(A) \cong \operatorname{hom}(S d \Delta, Y(H, A)) .
$$

This follows from classical ideas related to the Eilenberg-Zilber theorem or alternatively from the discussion that follows here. In general we will be able to prove that $\bar{H}(A)$ is homotopically equivalent to $\operatorname{hom}(S d \Delta, Y(H, A))$ provided that $\mathbf{B}$ is locally weakly Kan. (Recall that a simplicial set, $K$, is weakly Kan if any map $\Lambda^{i}[n] \rightarrow K$ for $0<i<n$ extends to one defined on $\Delta[n]$, where $\Lambda^{i}[n]$ is the $(n, i)$-horn that is the "union of the $n-1$ dimensional faces of $\Delta[n]$, except the $i^{\text {th }}$ face".) We must next turn our attention to a more detailed study of $S d \Delta$ and ordinal sum.

Given $p, q \in \mathbb{N}$, and the corresponding ordinals $[p],[q]$ in $\Delta$, we form their ordinal sum by concatenation

$$
[p]+[q]=\left\{0<1 \ldots<p<0^{\prime}<1^{\prime} \ldots<q^{\prime}\right\}
$$


where for convenience, we have put primes, " 1 ", on those elements corresponding to elements of $[q]$. We thus have, for instance, $[2]+[0] \cong[3]$; however when using ordinal sum within a simplicial context, functorial variation within $[p]+[q]$ can occur in $[p]$ or in $[q]$, but not at the divide so even if $[2]+[0] \cong[3]$, the result does not have a $d_{3}$-face, since there is no $[-1]$ to which we can map [0]. To help visualise $S d \Delta$, it pays to calculate at least $S d \Delta[1]$ and $S d \Delta[2]$ in detail.

Lemma 4.1. (i) The simplicial set $S d \Delta[n]$ can be decomposed into a collection of generalised prisms, $P(p)=\Delta[p] \times \Delta[n-p]$ where

$$
\left(\Delta[p] \times d_{0}\right) P(p)=\left(d_{p+1} \times \Delta[n-p-1]\right) P(p+1) .
$$

(ii) There is a natural embedding

$$
\operatorname{Sd} \Delta \rightarrow \operatorname{Subdiag}(\Delta \times \Delta),
$$

where in dimension $n$, Subdiag $(\Delta[n] \times \Delta[n])$ is the subsimplicial set of $\Delta[n] \times \Delta[n]$ under the diagonal, i.e. determined by those vertices $(i, j)$ with $i \leq j$.

Remarks. The two results making up this lemma indicate three important connections between $S d \Delta$ and other parts of Algebraic Topology.

(i) should be compared with the formula for the simplicial codiagonal functor often called $\nabla$ or $\bar{W}$, developed by Artin and Mazur, [5]. That functor from bisimplicial sets (groups, etc.) to simplicial sets (groups, etc.) is left adjoint to the functor induced by composition with ordinal sum. It is thus a Kan extension, given for a bisimplicial object $\left\{X_{p, q}\right\}$ by the coend

$$
(\nabla X)_{n}=\int^{[p],[q]} \Delta([p]+[q],[n]) \times X_{p, q},
$$

and usually interpreted in low dimensions via a diagram

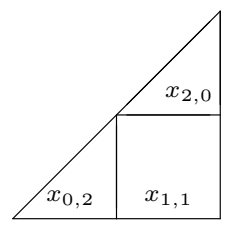

This same type of diagram occurs in numerous other contexts, for instance in Brown-Gilbert, [15].

(ii) is related to the constructions of "cellular approximations to the diagonal", (cf. Baues [8] p.16). The subdivision in the topological case is also used by Segal, [46].

Proof of 4.1. It suffices to note that the only maps in $\Delta([p]+[q],[n])$ that do not correspond to degenerate blocks or faces of higher dimensional blocks must have $p+q=n$ and be of the form

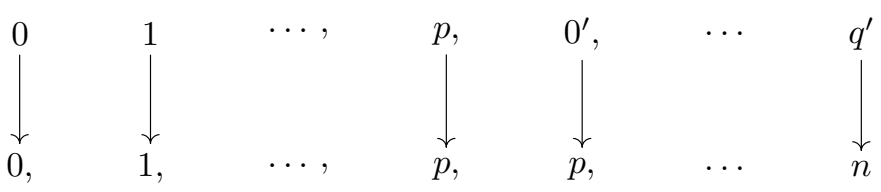

This gives normal forms for a sufficient set of non-degenerate prismal blocks glued together as claimed. These blocks can now be embedded into $\Delta[n] \times \Delta[n]$ as follows: If $\sigma:[p]+[q] \rightarrow[n]$, write $\sigma_{p}=\sigma \mid[p]$ and $\sigma_{q}=\sigma \mid[q]$; thus for instance in the 
above $\sigma_{p}(i)=i$ and $\sigma_{q}\left(i^{\prime}\right)=p+i$ (remember the primes " ' " are only there to distinguish the first and second parts of the decomposition).

This means that $\sigma$ induces a simplicial map

$$
\sigma: \Delta[p] \times \Delta[q] \rightarrow \Delta[n] \times \Delta[n]
$$

(in fact into $\operatorname{Subdiag}(\Delta[n] \times \Delta[n])$ ). It is routine to check that on the generating prisms of part (i) of this lemma, these various simplicial maps are compatible with the gluing face maps, thus giving the result.

Proposition 4.2 (Existence of Filling Schemes). Given any cosimplicial simplicial set, $K^{n}$, such that each $K^{n}$ is a weak Kan complex, then any map

$$
f: S d \Delta \rightarrow K
$$

extends to one defined on $\operatorname{Subdiag}(\Delta \times \Delta)$ and any two such extensions are homotopic.

Proof. As $S d \Delta[0] \cong \Delta[0]$, there is no difficulty in starting an induction on $n$. Assuming that such a map has been constructed on $\operatorname{Subdiag}(\Delta[n] \times \Delta[n])$, we can use cofaces to extend it over part of the next level up, namely that part corresponding to $\operatorname{Subdiag}\left(s k_{n} \Delta[n+1] \times s k_{n} \Delta[n+1]\right)$ where $s k_{n}$ is the $n$-skeleton functor. Within $\operatorname{Subdiag}(\Delta[n+1] \times \Delta[n+1])$, there are $C(n+1)$ simplices of dimension $2(n+1)$ where $C(k)$ is the $k^{\text {th }}$ Catalan number. These simplices, of course, correspond to paths of maximal length in the subdiagonal of the ordered set $[n+1] \times[n+1]$. Listing these paths and the face relations between them gives a graph on which is defined a natural depth function: For an illustrative example we take $n=3$; the subdiagonal is

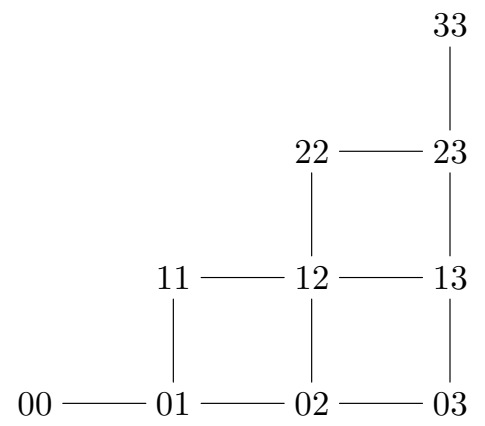

The 6 -simplex $(00,01,02,03,13,23,33)$ will be the starting point, whilst the 6 simplex with the diagonal as face is $(00,01,11,12,22,23,33)$. Each square represents a common face between two simplices, but this correspondence is not unique. Thus the square

$$
\begin{array}{ll}
11 & 12 \\
01 & 02
\end{array}
$$

links

$$
(00,01,02,12,13,23,33)
$$

with

$$
(00,01,11,12,13,23,33)
$$


as it provides a common $d_{2}$, namely

$$
(00,01,12,13,23,33)
$$

however this square also gives the link between

$$
(00,01,11,12,22,23,33)
$$

and

$$
(00,01,11,12,22,23,33) \text {. }
$$

The various maximal paths and their linking squares can be represented by the graph

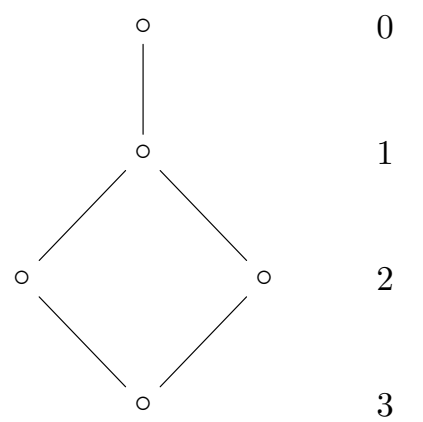

where the start vertex is at the top. The depth function measures the number of moves needed to get to that chain from the start 6 -simplex. List the simplices so that ones of depth $i$ are listed before those of depth $i+1$, for each $i$.

As we are assuming that the extension has already been made up to the $n^{\text {th }}$ level of $\Delta[n+1] \times \Delta[n+1]$, we are assuming known the value of the maps on all the simplices of $\operatorname{Subdiag}(\Delta[n+1] \times \Delta[n+1])$ that correspond to the inclusions of $\operatorname{Subdiag}(\Delta[n] \times \Delta[n])$ via coface maps. This knowledge interprets as knowledge of the extension on any subdiagram of the subdiagonal obtained by deleting an $i^{\text {th }}$ row and an $i^{\text {th }}$ column.

We start with a key observation, namely that to construct the overall extension it suffices to be able to extend $f$ over subdiagrams of the form

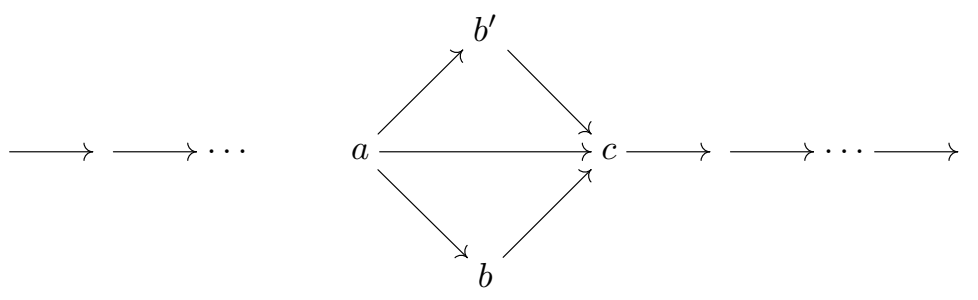

as each of the transitions is of this form. Thus we assume $f$ is already defined on the lower chain that goes via $a b c$ and try to extend it to the whole diagram thus obtaining it on the upper chain via $a b^{\prime} c$, which will be used to feed into a later step in the construction. This key step can be further broken down into checking that 
$f$ extends over all subdiagrams of form

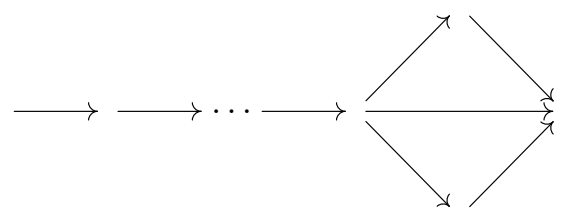

or

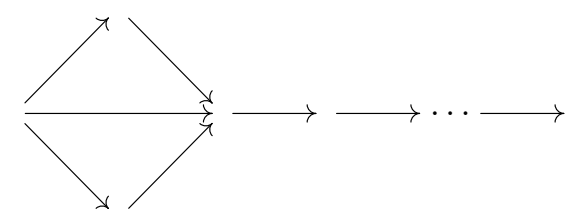

and then that it extends over all subdiagrams of a similar form but with the two adjacent 2-simplices replaced by $r$-simplices with their $d_{r-1}$ (or their $d_{1}$ ) faces combined. (Recall that these diagrams show partially ordered sets and we are working with an $f$ that to start with is known only on certain $(n+1)$-simplices in the nerve.) To illustrate the process, and before passing to the general case, we look at the start position,

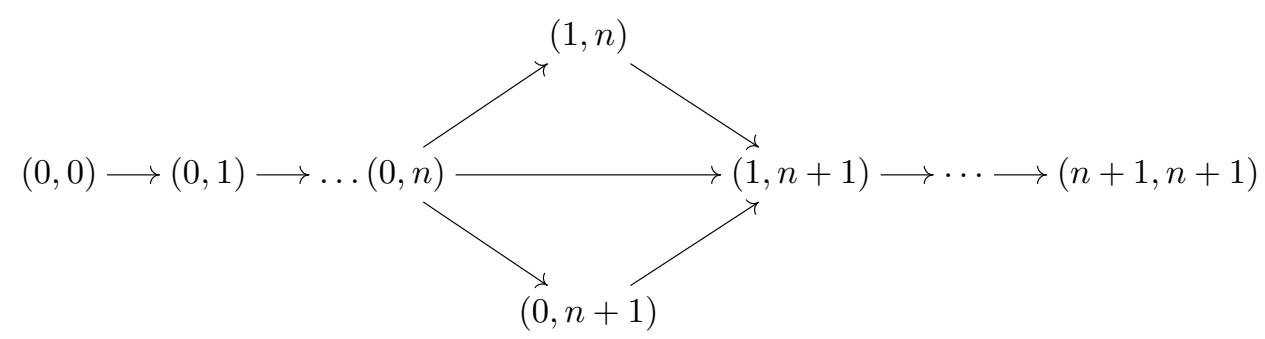

To start with $f$ is given on the $(n+1)$-simplices

$$
\begin{array}{ccccc}
(0,0), & \ldots, & (0, n+1) & & \\
(0,1), & \ldots, & (0, n+1), & (1, n+1) & \\
\cdots & & & \\
& & (0, n+1) & \ldots & (n+1, n+1)
\end{array}
$$

also on those simplices with $(1, n)$ replacing $(0, n+1)$ and, by inductive hypothesis, on any subdiagram that can be obtained by deleting an $i^{\text {th }}$ row and an $i^{\text {th }}$ column in the original diagram. We first attack the left-hand "half" of the diagram ending with $(1, n+1)$, to try to extend $f$ over the resulting $(n+2)$-simplices which share a common face.

We assume as an induction hypothesis that provided the length of the lead-in string is less than $n$, then an extension exists. We find that in the $(n+2)$-simplex

$$
(0,0), \ldots,(0, n),(0, n+1),(1, n+1),
$$

we know already the value of $f$ on all faces except $d_{1}$ and $d_{n+1}$. We note $d_{1}$ is the face

$$
(0,0),(0,2), \ldots(0, n),(0, n+1),(1, n+1)
$$

and by inductive hypothesis this causes no difficulties. 
This leaves a $\Lambda^{n+1}[n+2]$ over which it is easy to extend $f$. Now in the simplex

$$
(0,0), \ldots,(0, n),(1, n),(1, n+1),
$$

we know $f$ on $d_{n+1}$ as that face is shared with the simplex we have just handled. We know $f$ on $d_{i}, 2 \leq i<n$, as before, since we know $f$ on $S d\left(s k_{n} \Delta\right)$. We know $f$ on $d_{0}$, since this forms part of the prism, $\Delta[1] \times \Delta[n]$, within $S d \Delta[n+1]$ and $d_{1}$ can be handled as before by our inductive hypothesis. This leaves $f$ known on a $\Lambda^{n}[n+2]$, which can be filled using the fact that $K^{n}$ is weakly Kan. (The case $n=0$ does not cause any difficulty as is easily checked.)

Building on from this to extend $f$ over the two $(2 n+2)$ simplices is now easy. The neatest way to do this is via an inductive argument up the "tail" between $(1, n+1)$ and say $(i, n+1)$.

The plan sketched earlier now suggests extending $f$ over

$$
(0,0), \ldots,(0, n-1),(1, n-1),(1, n),(1, n+1) .
$$

Our previous calculation gives us $d_{n}$. For $i$ in the range $2 \leq i<n-1$, the boundary of $S d \Delta[n+1]$ gives us an extension. As before $d_{1}$ can be handled by inductive hypothesis and $d_{0}$ is given. This leaves a $\Lambda^{n-1}[n+2]$ on which $f$ is known and the extension proceeds as planned. Extending $f$ over the $(2 n+2)$-simplex obtained by adding the tail causes no problems.

Returning to the other simplex

$$
(0,0), \ldots(0, n),(1, n),(2, n),(2, n+1), \ldots,(n+1, n+1),
$$

it is simplest to view this in the other direction first extending $f$ over

$$
(0, n),(1, n),(2, n),(2, n+1) \ldots(n+1, n+1),
$$

then adding a "head" string from $(0,0)$ to $(0, n)$. We omit the details.

The extension of $f$ over the $2 n+2$ simplices of $\operatorname{Subdiag}(\Delta[n+1] \times \Delta[n+1])$ continues in this way. If the path giving the simplex has a "new part" at vertex $(i, j)$ in its $k^{\text {th }}$ position

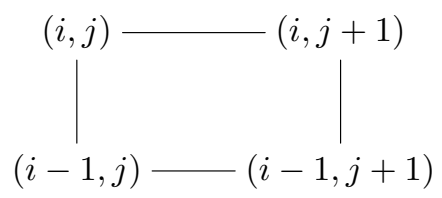

then provided $k \leq n+1$, we can extend $f$ using a $\Lambda^{k-1}[n+2]$ over which $f$ is either given $a b$ initio, given by the extension already made to level $n$ in the cosimplicial dimension, or can be extended by application of the inductive hypothesis. (If $k \geq n+1$, then operation using the "tail" rather than the start is used.)

The filling scheme extends over all simplices at a given depth $k$ before passing to the next. This ensures that enough faces have already been constructed for the extension to work and makes certain that no conflict occurs with a face being needed for filling whilst it has already been used by another part of the process.

This completes the extension process. Any two such extensions will be homotopic as the extension schema can also be used to extend any map defined from $S d \Delta$ to $\mathbf{S}(\Delta[1], K)$ to one defined on $\operatorname{Subdiag}(\Delta \times \Delta)$. 
The final points to make are that we have, as we progressed, effectively constructed a proof scheme that allows the proof of the inductive hypothesis concerning the lengths of "head" or "tail" over which extensions can be made. Moreover, nowhere in the above did it prove necessary to fill a $\Lambda^{0}[k]$ or a $\Lambda^{k}[k]$ for any $k$.

Corollary 4.3. Given a cosimplicial simplicial set $Y$ such that each $Y^{n}$ is a weak Kan complex, there is a map

$$
\operatorname{hom}(\operatorname{Sd} \Delta, Y) \rightarrow \operatorname{hom}(\operatorname{Subdiag}(\Delta \times \Delta), Y),
$$

which is left inverse to the restriction map, i.e. composition with the restriction map gives the identity on hom $(S d \Delta, Y)$. Moreover any two such maps are homotopic.

This corollary applied to $Y(H, A)$ gives a map

$$
\operatorname{Coh}(F, G) \times \operatorname{Coh}(G, H) \rightarrow \operatorname{hom}(\operatorname{Subdiag}(\Delta \times \Delta), Y(F, H)),
$$

and by composition with the restriction to the diagonal a choice of "composition" map:

$$
c_{F, H}^{G}: \operatorname{Coh}(F, G) \times \operatorname{Coh}(G, H) \rightarrow \operatorname{Coh}(F, H) .
$$

Unfortunately, with all the choices we have made, it is extremely unlikely that this "composition" will be associative, and in general it will not be. On the other hand it $i s$ as coherently associative as we might want; e.g. for any $H$, the square

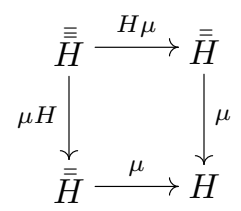

will be homotopy coherent and so for $F, G, H, K$ being $S$-functors from $\mathbf{A}$ to $\mathbf{B}$, the two composites

$$
\operatorname{Coh}(F, G) \times \operatorname{Coh}(G, H) \times \operatorname{Coh}(H, K) \longrightarrow \operatorname{Coh}(F, K)
$$

will be homotopic. The proof of this involves the triple ordinal sum $[p]+[q]+[r]$, a triple subdivision of $\Delta$ and a modification of the filling scheme of 4.2 to allow for the third direction in $\Delta \times \Delta \times \Delta$.

This means that $\operatorname{Coh}(\mathbf{A}, \mathbf{B})$, for $\mathbf{A}$ small and $\mathbf{B}$ locally weakly Kan, has the structure of what one might call an $H$-category, i.e. a homotopy coherent model of the theory of categories. As a result, on taking $\pi_{0} \operatorname{Coh}(F, G)=\operatorname{coh}(F, G)$, we get an actual category $\operatorname{coh}(\mathbf{A}, \mathbf{B})$ (which is essentially that introduced by Batanin, [7] for the case that $\mathbf{B}$ is locally Kan).

We sum this up in the following:

Theorem 4.4. If $\boldsymbol{A}$ is a small $S$-category and $\mathbf{B}$ is a locally weakly Kan $S$ category, then there are simplicial composition maps

$$
c_{F, H}^{G}: \operatorname{Coh}(\mathbf{A}, \mathbf{B})(F, G) \times \operatorname{Coh}(\mathbf{A}, \mathbf{B})(G, H) \rightarrow \operatorname{Coh}(\mathbf{A}, \mathbf{B})(F, H),
$$

which are well defined up to homotopy and are coherently associative. 
Remark. The passage via $\overline{\bar{H}}$ and a "multiplication map" to $\bar{H}$ may not seem to be "geometric" enough for some readers; however it is easy to obtain the composite in an alternative way.

$$
\begin{aligned}
C o h(\mathbf{A}, \mathbf{B})(F, G) & =\int_{A, A^{\prime}} \mathbf{S}\left(\hat{\mathbf{A}}\left(A, A^{\prime}\right), \mathbf{B}\left(F A, G A^{\prime}\right)\right) \\
& \cong \int_{A, A^{\prime}} \mathbf{S}\left(\int^{[m]} \Delta[m] \times X\left(A, A^{\prime}\right)_{m}, \mathbf{B}\left(F A, G A^{\prime}\right)\right)
\end{aligned}
$$

and

$$
\operatorname{Coh}(\mathbf{A}, \mathbf{B})(G, H) \cong \int_{A^{\prime \prime}, A^{\prime \prime \prime}} \mathbf{S}\left(\int^{[n]} \Delta[n] \times X\left(A^{\prime \prime}, A^{\prime \prime \prime}\right)_{n}, \mathbf{B}\left(G A^{\prime \prime}, H A^{\prime \prime \prime}\right)\right)
$$

Restricting to composable pairs gives a map from

$$
\operatorname{Coh}(\mathbf{A}, \mathbf{B})(F, G) \times \operatorname{Coh}(\mathbf{A}, \mathbf{B})(G, H)
$$

to

$$
\int_{A, A^{\prime}, A^{\prime \prime}} \mathbf{S}\left(\int^{[m],[n]} X\left(A, A^{\prime \prime}\right)_{m+n+1} \times \Delta[m] \times \Delta[n], \mathbf{B}\left(F A, G A^{\prime}\right) \times \mathbf{B}\left(G A^{\prime}, H A^{\prime \prime}\right)\right)
$$

and thus by composition to

$$
\int_{A, A^{\prime \prime}} \mathbf{S}\left(\int^{[m],[n]} X\left(A, A^{\prime \prime}\right)_{m+n+1} \times \Delta[m] \times \Delta[n], \mathbf{B}\left(F A, H A^{\prime \prime}\right)\right),
$$

but this is $\operatorname{hom}(S d \Delta, Y(F, H))$ as before and the map is the same.

We finish this section by examining the relation of the coherent "composition" that has just been studied and the natural composition

$$
\mathbf{B}^{\mathbf{A}}(F, G) \times \mathbf{B}^{\mathbf{A}}(G, H) \rightarrow \mathbf{B}^{\mathbf{A}}(F, H) .
$$

We noted in section 3 that the augmentation of the $\hat{\mathbf{A}}\left(A, A^{\prime}\right)$ defined the natural map from $G$ to $\bar{G}$ and hence the natural map from $\mathbf{B}^{\mathbf{A}}(F, G)$ to $C o h(\mathbf{A}, \mathbf{B})(F, G)$. We thus have a diagram

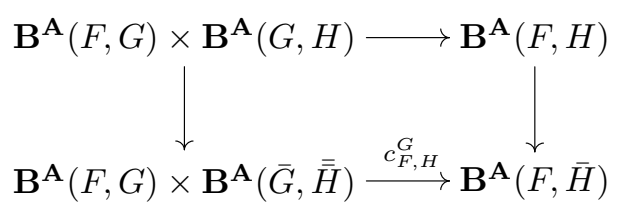

which it might be hoped would be commutative, at least up to homotopy. We noted that the homotopy class of $c_{F, H}^{G}$ was well defined, so to verify the commutativity of this diagram up to homotopy, it suffices to produce a choice of $c_{F, H}^{G}$ that extends the natural composition at the top of the square. The earlier results on the passage from $\hat{\mathbf{A}}\left(A, A^{\prime}\right)$ to $\mathbf{A}\left(A, A^{\prime}\right)$ allow one to identify the natural maps within $\operatorname{Coh}(\mathbf{A}, \mathbf{B})(F, G)$ and within $\operatorname{Coh}(\mathbf{A}, \mathbf{B})(G, H)$ (cf. 3.9). Feeding this information into the filling scheme, one finds that canonical extensions exist for these as they are constant on simplices of $\Delta$.

Thus when constructing the map from

$$
\operatorname{hom}(S d \Delta, Y(F, H))
$$


to

$$
\operatorname{hom}(\operatorname{Subdiag}(\Delta \times \Delta), Y(F, H)) \text {, }
$$

the mapping of simplices corresponding to pairs of natural transformations causes no difficulty. Agreeing that this is done first, one can construct the rest of the mapping using the filling scheme as given. A slight modification of this argument shows that one can choose the restriction of $c_{F, H}^{G}$ to the subsimplicial sets, $\mathbf{B}^{\mathbf{A}}(F, G) \times \operatorname{Coh}(\mathbf{A}, \mathbf{B})(G, H)$ and $\operatorname{Coh}(\mathbf{A}, \mathbf{B})(F, G) \times \mathbf{B}^{\mathbf{A}}(G, H)$, so as to coincide with the obvious compositions.

$$
\mathbf{B}^{\mathbf{A}}(F, G) \times \mathbf{B}^{\mathbf{A}}(G, \bar{H}) \rightarrow \mathbf{B}^{\mathbf{A}}(F, \bar{H})
$$

and

$$
\mathbf{B}^{\mathbf{A}}(F, \bar{G}) \times \mathbf{B}^{\mathbf{A}}(\bar{G}, \bar{H}) \rightarrow \mathbf{B}^{\mathbf{A}}(F, \bar{H}),
$$

as in each case, on one of the two directions, the resulting simplices correspond to constant simplicial maps.

We summarise this in the following.

Proposition 4.5. The coherent composition $c_{F, H}^{G}$ of 4.4 can be chosen to extend the natural composition defined on

$$
\mathbf{B}^{\mathbf{A}}(F, G) \times \operatorname{Coh}(\mathbf{A}, \mathbf{B})(G, H) \cup \operatorname{Coh}(\mathbf{A}, \mathbf{B})(F, G) \times \mathbf{B}^{\mathbf{A}}(G, H) .
$$

The above argument can be seen to depend implicitly on the identification of $\mathbf{B}^{\mathbf{A}}(F, G)$ with $\operatorname{hom}(*, Y(F, G))$, where $*$ is the constant cosimplicial simplicial set with value $\Delta[0]$. In the case of a locally Kan $S$-category $\mathbf{B}$, rather than a locally weakly Kan one, this allows a simpler proof of the above result using certain

diagrams in the category of cosimplicial simplicial sets and their realisations in the category of cosimplicial spaces.

\section{Coherent extensions}

The standard treatment of (right) Kan extensions takes functors $K: \mathbf{A} \rightarrow \mathbf{B}, F$ : $\mathbf{A} \rightarrow \mathbf{C}$ and asks for a universal solution $R_{K} F: \mathbf{B} \rightarrow \mathbf{C}$ to extending $F$ along $K$; i.e. if $G: \mathbf{B} \rightarrow \mathbf{C}$ is any functor, one wants

$$
\operatorname{Nat}(G K, F) \cong \operatorname{Nat}\left(G, R_{K} F\right) .
$$

This then leads to an end formula for $R_{K} F$

$$
R_{K} F(B)=\int_{A} \overline{\mathbf{C}}(\mathbf{B}(B, K A), F A)
$$

(see Mac Lane, [38]).

Attempts to generalise this to the simplicial coherence context are complicated by the choices available. Clearly one can replace "Nat" by "Coh", but should isomorphism be replaced by homotopy equivalence, should this be natural, in which direction should it go, etc., so to start our investigation of "coherent Kan extensions", we will study the "obvious" construction of a right coherent extension

$$
R_{K} F(B)=\oint_{A} \overline{\mathbf{C}}(\mathbf{B}(B, K A), F A)
$$

using a coherent end where $\mathbf{A}, \mathbf{B}, \mathbf{C}$, and $F$ and $K$ are all $S$-enriched. This gives constructions related to the bar and cobar resolutions (Meyer, [41], [42]) and to 
ideas exploited by Dwyer and Kan, [25], Heller, [33] and others. There is a similar formula for a left extension

$$
L_{K} F(B)=\oint^{A} \mathbf{B}(K A, B) \bar{\otimes} F A .
$$

Our aim is to study the properties of these to show that it is justifiable to claim that, as these satisfy suitable coherent versions of standard theorems, they can safely be considered to be coherent Kan extensions.

We thus fix the above data $: K: \mathbf{A} \rightarrow \mathbf{B}, \mathbf{F}: \mathbf{A} \rightarrow \mathbf{C}$ with $\mathbf{C}$ complete or cocomplete as necessary and $R_{K} F$ and $L_{K} F$ defined as in (1) and (2) above.

Proposition 5.1. Let $G: \mathbf{B} \rightarrow \mathbf{C}$ be any $S$-functor. Then there is a natural isomorphism

$$
\operatorname{Coh}(\mathbf{A}, \mathbf{C})(G K, F) \cong \mathbf{C}^{\mathbf{B}}\left(G, R_{K} F\right)
$$

(dually:

$$
\left.\operatorname{Coh}(\mathbf{A}, \mathbf{C})(F, G K) \cong \mathbf{C}^{\mathbf{B}}\left(L_{K} F, G\right)\right) .
$$

Proof. (This is similar to that of 3.3.)

$$
\begin{aligned}
\mathbf{C}^{\mathbf{B}}\left(G, R_{K} F\right) & =\int_{B} \mathbf{C}\left(G B, R_{K} F B\right) \\
& \cong \int_{B} \mathbf{C}\left(G B, \int_{A^{\prime}, A^{\prime \prime}} \overline{\mathbf{C}}\left(\hat{\mathbf{A}}\left(A^{\prime}, A^{\prime \prime}\right), \overline{\mathbf{C}}\left(\mathbf{B}\left(B, K A^{\prime}\right), F A^{\prime \prime}\right)\right)\right) \\
& \cong \int_{A^{\prime}, A^{\prime \prime}} \mathbf{S}\left(\hat{\mathbf{A}}\left(A^{\prime}, A^{\prime \prime}\right), \int_{B} \mathbf{S}\left(\mathbf{B}\left(B, K A^{\prime}\right), \mathbf{C}\left(G B, F A^{\prime \prime}\right)\right)\right) \\
& \cong \int_{A^{\prime}, A^{\prime \prime}} \mathbf{S}\left(\hat{\mathbf{A}}\left(A^{\prime}, A^{\prime \prime}\right), \mathbf{C}\left(G K A^{\prime}, F A^{\prime \prime}\right)\right) \quad \text { by Yoneda } \\
& \cong \operatorname{Coh}(\mathbf{A}, \mathbf{C})(G K, F) .
\end{aligned}
$$

The dual proof proves the dual statement.

\section{EXAMPLES AND REMARKS}

1. The case where $K$ is the identity functor reduces to the definition of $\bar{F}$ (resp: $\underline{F})$ in section 4 . The properties of the construction, $\underline{F}$, for the case $\mathbf{C}=$ Top are considered in detail in [23].

2. If one uses $\hat{\mathbf{A}}^{*}\left({ }_{-},-\right)$instead of $\hat{\mathbf{A}}\left({ }_{-},-\right)$as the indexation, the construction of $L_{K} F$ is related to that of the $\pi^{*} F$ of Segal, [46]. Replacing $\mathbf{A}$ and $\mathbf{B}$ by the corresponding Top-enriched categories via the geometric realisation of their homobjects, $\pi^{*} F$ corresponds to $L_{K} F$ in the case $K^{o p}=\pi^{o p}: \mathbf{A}^{o p} \rightarrow \mathbf{B}^{o p}$.

3. Recall from [10] that the Bousfield-Kan homotopy limit can be extended to one defined at the simplicially enriched level, thus covering the case of homotopy coherent diagrams. Explicitly let $F: \mathbf{A} \rightarrow \mathbf{C}$ be given and define $H_{A}: \mathbf{A} \rightarrow \mathbf{S}$ by $H_{A}(A)=\operatorname{Diag} Y(A)$, where

$$
Y(A)_{n,-}=\coprod_{A_{0}, \ldots, A_{n}} \mathbf{A}\left(A_{0}, A_{1}\right) \times \ldots \times \mathbf{A}\left(A_{n-1}, A_{n}\right) \times \mathbf{A}\left(A_{n}, A\right) ;
$$

then define

$$
\operatorname{holim} F=\int_{A} \overline{\mathbf{C}}\left(H_{A}(A), F A\right)
$$


and dually

$$
\text { hocolim } F=\int^{A} H_{A^{o p}}(A) \bar{\otimes} F A .
$$

If $\mathbf{A}$ is an ordinary small category considered with the trivial $S$-category structure, then these are precisely the Bousfield-Kan homotopy limit and colimit since in that case $H_{A}(A)=N e r(\mathbf{A} / A)$.

Proposition 5.2. Let $F: \mathbf{A} \rightarrow \mathbf{C}$ be an $S$-functor where $\mathbf{C}$ is a complete (resp. cocomplete) $S$-category. Then taking $K: \mathbf{A} \rightarrow \mathbf{I}$ to be the unique $\mathbf{S}$-functor to the $S$ category with one object, $*$, and one non-degenerate morphism, the Bourn-Cordier homotopy limit, holimF, (resp. the homotopy colimit, hocolimF), is isomorphic to $R_{K} F$ (resp. $L_{K} R$ ).

Proof. We have

$$
\begin{aligned}
R_{K} F(*) & =\oint_{A} \overline{\mathbf{C}}(\Delta[0], F A) \\
& =\int_{A, A^{\prime}} \overline{\mathbf{C}}\left(\hat{\mathbf{A}}\left(A, A^{\prime}\right), \overline{\mathbf{C}}\left(\Delta[0], F A^{\prime}\right)\right) \\
& =\int_{A^{\prime}} \overline{\mathbf{C}}\left(\int^{A} \hat{\mathbf{A}}\left(A, A^{\prime}\right), F A^{\prime}\right)
\end{aligned}
$$

but

$$
\hat{\mathbf{A}}\left(A, A^{\prime}\right)_{n}=\coprod_{A_{0}, \ldots, A_{n}} \mathbf{A}\left(A_{0}, A_{1}\right)_{n} \times \ldots \times \mathbf{A}\left(A_{n-1}, A_{n}\right)_{n} \times \mathbf{A}\left(A_{n}, A^{\prime}\right)_{n}
$$

so

$$
\int^{A} \hat{\mathbf{A}}\left(A, A^{\prime}\right)=\operatorname{colim}_{A} \hat{\mathbf{A}}\left(A, A^{\prime}\right) \cong H_{A}\left(A^{\prime}\right) .
$$

The result follows.

4. If $\mathbf{A}$ is any small category, then homotopy coherent diagrams of type $\mathbf{A}$ in the $S$-category $\mathbf{C}$ correspond, by [16], to $S$-functors from $S(\mathbf{A})$ to $\mathbf{C}$, where $S(\mathbf{A})$ is the $S$-category constructed using the "free-forget" resolution as in [16]. There is an augmentation $S$-functor

$$
K: S(\mathbf{A}) \rightarrow \mathbf{A}
$$

where $\mathbf{A}$ is given the trivial $S$-category structure. If $\mathbf{C}$ is a complete (resp. cocomplete) $S$-category and $F: S(\mathbf{A}) \rightarrow \mathbf{C}$ is a homotopy coherent diagram of type $\mathbf{A}$, then $R_{K} F: \mathbf{A} \rightarrow \mathbf{C}$ (resp. $L_{K} F: \mathbf{A} \rightarrow \mathbf{C}$ ) is an actual functor. In [23], we used the term "rectification" for this functor although our definition there is apparently different.

It is interesting to note that in general it would seem difficult, if not impossible, to express a coherent extension as a homotopy limit (or colimit) of a functor with domain some "comma $S$-category". The problem is to know how such a "comma $S$-category" should be formed. In this last case however such a reduction is possible.

Proposition 5.3. Let $\mathbf{A}$ be a small category, $K: S(\mathbf{A}) \rightarrow \mathbf{A}$, the augmentation $S$-functor and $F: S(\mathbf{A}) \rightarrow \mathbf{C}$ a homotopy coherent diagram in a complete (resp. cocomplete) $S$-category, C. For $B$ an object of $\mathbf{A}$, one has $R_{K} F(B)=\operatorname{holim} F S\left(\delta_{B}\right)$ (resp. $L_{K} F(B)=$ hocolim $F S\left(\delta_{B}\right)$ ) where $\delta_{B}$ is the canonical functor from the comma category $B \downarrow \mathbf{A}$ to $\mathbf{A}$ (resp. from the comma category $\mathbf{A} \downarrow B$ to $\mathbf{A}$ ). 
Proof. Let $S\left(\delta_{B}\right): S(B \downarrow \mathbf{A}) \rightarrow S(\mathbf{A})$; then for $F: S(\mathbf{A}) \rightarrow \mathbf{C}$, one has

$$
\begin{array}{r}
\operatorname{holimFS}\left(\delta_{B}\right)=\int_{(A, f)} \mathbf{C}\left(\int^{[n]} \Delta[n] \times \coprod S(B \downarrow \mathbf{A})\left(\left(A_{0}, f_{0}\right),\left(A_{1}, f_{1}\right)\right)\right. \\
\left.\times \ldots \times S(B \downarrow \mathbf{A})\left(\left(A_{n}, f_{n}\right),(A, f)\right), F A\right),
\end{array}
$$

where the indexation of the coproduct is over all $\left(A_{0}, f_{0}\right), \ldots,\left(A_{n}, f_{n}\right)$, since of course $F S\left(\delta_{B}\right)(A, f)=F A$. The end formula for $R_{K} F(B)$ involves a similar term but with

$$
\coprod_{A_{0}, \ldots, A_{n}} \mathbf{A}\left(B, A_{0}\right) \times S(\mathbf{A})\left(A_{0}, A_{1}\right) \times \ldots \times S(\mathbf{A})\left(A_{n-1}, A_{n}\right)
$$

and the each term splits up as a disjoint union of terms of the form

$$
S(B \downarrow \mathbf{A})\left(\left(A_{0}, f_{0}\right),\left(A_{1}, f_{1}\right)\right) \times \ldots \times S(B \downarrow \mathbf{A})\left(\left(A_{n}, f_{n}\right)(A, f)\right) .
$$

It is now easily checked that the result holds.

\section{Coherent extensions With locally Kan codomain}

In section 2, we showed that the theory of coherent ends and coends was especially nice when the codomain category was locally Kan. This, of course, has consequences for the theory of coherent extensions.

Proposition 6.1. If $\mathbf{C}$ is a complete cotensored locally Kan $S$-category, $K: \mathbf{A} \rightarrow$ $\mathbf{B}$ and $F: \mathbf{A} \rightarrow \mathbf{C}$ are $S$-functors and $R_{K} F$ is defined as above then there is a homotopy equivalence

$$
\operatorname{Coh}(\mathbf{A}, \mathbf{C})(G K, F) \simeq \operatorname{Coh}(\mathbf{B}, \mathbf{C})\left(G, R_{K} F\right) .
$$

The proof of this is a combination of 5.1 and the following lemma, which should be compared with Corollary 3.7.

Lemma 6.2. With the same data as 6.1, the natural map

$$
\mathbf{C}^{B}\left(G, R_{K} F\right) \rightarrow \operatorname{Coh}(\mathbf{B}, \mathbf{C})\left(G, R_{K} F\right)
$$

is a homotopy equivalence.

Proof. We know

$$
\begin{aligned}
\operatorname{Coh}(\mathbf{B}, \mathbf{C})\left(G, R_{K} F\right) & =\oint_{B} \mathbf{C}\left(G B, \oint_{A} \mathbf{C}(\mathbf{B}(B, K A), F A)\right) \\
& \cong \oint_{A} \oint_{B} \mathbf{S}(\mathbf{B}(B, K A), \mathbf{C}(G B, F A))
\end{aligned}
$$

and

$$
\mathbf{C}^{B}\left(G, R_{K} F\right)=\oint_{A} \int_{B} \mathbf{S}\left(\mathbf{B}(B, K A), \mathbf{C}\left(G B, F A^{\prime}\right)\right) .
$$

We set

$$
\begin{aligned}
T_{0}\left(A, A^{\prime}\right) & =\int_{B} \mathbf{S}\left(\mathbf{B}(B, K A), \mathbf{C}\left(G B, F A^{\prime}\right)\right) \\
& \cong \mathbf{C}\left(G K A, F A^{\prime}\right)
\end{aligned}
$$


and

$$
\begin{aligned}
T_{1}\left(A, A^{\prime}\right) & =\oint_{B} \mathbf{S}\left(\mathbf{B}(B, K A), \mathbf{C}\left(G B, F A^{\prime}\right)\right) \\
& \cong \int_{B^{\prime}, B^{\prime \prime}} \mathbf{S}\left(\hat{\mathbf{B}}\left(B^{\prime}, B^{\prime \prime}\right) \times \mathbf{B}\left(B^{\prime \prime}, K A\right), \mathbf{C}\left(G B^{\prime}, F A^{\prime \prime}\right)\right) \\
& \cong \int_{B^{\prime}} \mathbf{S}\left(\hat{\mathbf{B}}\left(B^{\prime}, K A\right), \mathbf{C}\left(G B^{\prime}, F A^{\prime}\right)\right) .
\end{aligned}
$$

There is a natural homotopy equivalence, induced by the augmentation $d_{0}: \hat{\mathbf{B}}\left(B^{\prime},{ }_{-}\right)$ $\rightarrow \mathbf{B}\left(B^{\prime},{ }_{-}\right)$and its homotopy inverse $s_{-1}$, which gives a homotopy equivalence from $T_{0}\left(A, A^{\prime}\right)$ to $T_{1}\left(A, A^{\prime}\right)$. If $\mathbf{C}$ is locally Kan, then both $T_{0}$ and $T_{1}$ take Kan values and 2.4 finishes the proof.

Thus if $\mathbf{C}$ is locally Kan, the coherent extension construction performs exactly as one would hope a homotopy coherent Kan extension would perform. There is a dual form of 6.1 involving $L_{K} F$ which we will leave the reader to state and prove.

We pointed out in section 4 that if we defined

$$
\operatorname{coh}(\mathbf{A}, \mathbf{C})(F, G)=\pi_{0} \operatorname{Coh}(\mathbf{A}, \mathbf{C})(F, G),
$$

we obtained a category.

Corollary 6.3. If $C$ is a complete cotensored locally Kan $\mathbf{S}$-category, $K: \mathbf{A} \rightarrow \mathbf{B}$ and $F: \mathbf{A} \rightarrow \mathbf{C}$ are $S$-functors and $R_{K} F$ is as above then

$$
\operatorname{coh}(\mathbf{A}, \mathbf{C})(G K, F) \cong \operatorname{coh}(\mathbf{B}, \mathbf{C})\left(G, R_{K} F\right) .
$$

(There is a dual form involving $L_{K} F$.)

This result, or rather a special case of it has been noted several times by other authors. If $\mathbf{A}=S(\mathbf{I}), \mathbf{B}=S(\mathbf{J})$, where $S$ is the construction studied by the first author in [16], then $\operatorname{coh}(\mathbf{A}, \mathbf{C}) \cong H o\left(\mathbf{C}^{\mathbf{I}}\right)$ by the extension of Vogt's theorem, [21]. Thus a functor $K: \mathbf{I} \rightarrow \mathbf{J}$ induces $S(K): S(\mathbf{I}) \rightarrow S(\mathbf{J})$ and if $\mathbf{C}$ is as above, we have a functor,

$$
H o\left(\mathbf{C}^{\mathbf{K}}\right): H o\left(\mathbf{C}^{\mathbf{J}}\right) \rightarrow H o\left(\mathbf{C}^{\mathbf{I}}\right),
$$

and $R_{K}$ induces a right adjoint to this. In other words the $R_{K}$ construction yields a "homotopy Kan extension" as introduced by Anderson, [2] and studied by Heller [33], [34]. Dwyer and Kan, [25], have also given such a construction. Heller [34] has argued convincingly that the "hyper-functor", which to a small category I assigns $H o\left(\mathbf{C}^{\mathbf{I}}\right)$ and to a functor $K$ assigns the induced functor together with homotopy Kan extensions, makes up the essential structure of a homotopy theory. It is of interest to note that Grothendieck in [31] argues in a parallel way for studying the categories $D\left(\mathbf{A}^{\mathbf{I}}\right)$ and the induced functors with the corresponding extensions to give a more complete version of Verdier's theory of derived categories. We feel that the categories $H o\left(\mathbf{C}^{\mathbf{I}}\right)$ and $D\left(\mathbf{A}^{\mathbf{I}}\right)$, as they are, roughly speaking, the $\pi_{0}$ level of a much richer structure, only reflect a small amount of the structure that is there and that, if a theory can be developed that can handle the corresponding structures $\operatorname{Coh}(\mathbf{A}, \mathbf{C})$ etc., then this may provide a very significant tool for the future development of this area of abstract homotopy theory.

Another application of these coherent Kan extensions is in the theory of generalised derived functors. This aspect is already implicit in the brief discussion 
above of Grothendieck's views on the development of the theory of derived categories. Here we will mention another link, briefly sketching the way that a trivial case of the $L_{K}$-construction yields a theory of derived functors that generalises the comonad derived functors considered by Quillen, [45], Tierney and Vogel, [49], André, [3] and Ulmer, [50]. They thus, among other things, generalise classical derived functors. The basic idea for the construction used is taken from the unpublished thesis of P. Gardener [29], which generalised results of Alan Robinson, [44].

We take as given a category $\mathbf{B}$ with an initial object $I$ and a subcategory $\mathbf{A}$ of $\mathbf{B}$. (The objects of $\mathbf{A}$ will be called the models and play here the rôle of the projective objects in the classical theory of derived functors.) This category $\mathbf{A}$ will be the domain of a functor $F$ with codomain a category of "based sets with structure", which we will tacitly assume most of the time is just the category of pointed sets. Finally we assume $I$ is a model. Given this data and an object $B$ of $\mathbf{B}$, form up a category $\tau(B, F)$ having as objects pairs $(f, x)$ where $f: M \rightarrow B$ is a morphism of $\mathbf{B}$ with domain in $\mathbf{A}$ and $x \in F M$. The morphisms of $\tau(B, F)$ from $\left(f_{1}, x_{1}\right)$ to $\left(f_{2}, x_{2}\right)$ correspond to A-morphisms $\xi: M_{1} \rightarrow M_{2}$ so that

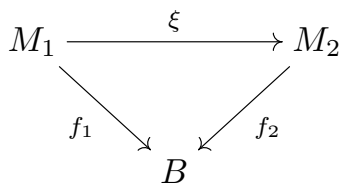

commutes and $F \xi\left(x_{1}\right)=x_{2}$. Denoting the nerve of $\tau(B, F)$ by $N \operatorname{er}(\tau(B, F))$, the candidates for the derived functors of $F$ evaluated at $B$ are the homotopy groups, $\pi_{n}(N \operatorname{er}(\tau(B, F)))$.

These functors behave well; for instance the functor $\pi_{0}(\operatorname{Ner}(\tau(-, F)))$ is just the Kan extension of $F$ along the inclusion of $\mathbf{A}$ into $\mathbf{B}$.

If it is assumed that $\mathbf{B}$ is an abelian category with enough projectives and $\mathbf{A}$ is the full subcategory of projectives, then if $F$ has codomain an abelian category, these "derived functors" are isomorphic to the classical derived functors of Cartan and Eilenberg. Other less classical situations can also be brought into this description. In particular, if $\mathbf{B}$ is a category on which we are given a comonad, $G$, and $\mathbf{A}$ is the full subcategory of the $G$-free objects then for $F$ having abelian domain, these derived functors coincide with those of Barr and Beck, [6]. Finally we note that Gardener, [29], proves that taking $\mathbf{B}$ to be the category of based topological spaces and $\mathbf{A}$, the full subcategory of spaces homotopy equivalent to finite discrete spaces, then $\pi_{n}\left(\operatorname{Ner}\left(\tau\left(B, \pi_{0}\right)\right)\right) \cong \pi_{n} B$, so the homotopy groups are also derived functors in this sense.

Given this situation with data $(\mathbf{B}, \mathbf{A}, F)$, we replace $F$ by the functor $K(F, O)$ : $\mathbf{A} \rightarrow \mathbf{S}_{*}$ where $K(F M, O)$ will be the constant pointed simplicial set on the pointed set $F M$. Abusing notation, we will write $F$ for this functor as well, and similarly consider $\mathbf{A}$ and $\mathbf{B}$ as having trivial $S$-enrichment.

Proposition 6.4. There is a natural isomorphism

$$
L_{K} F(B) \cong N e r(\tau(B, F))
$$

where $K: \mathbf{A} \rightarrow \mathbf{B}$ is the inclusion. 
Proof. We note

$$
\begin{aligned}
L_{K} F(C) & =\oint^{M} \mathbf{B}(K M, B) \bar{\otimes} F M \\
\cong & \int^{M, M^{\prime}} \hat{\mathbf{A}}\left(M^{\prime}, M\right) \times \mathbf{B}(K M, B) \times F M^{\prime} \\
\cong & \int^{[n]} \Delta[n] \times \coprod \mathbf{A}\left(M_{0}, M_{1}\right) \times \ldots \times \mathbf{A}\left(M_{n-1}, M_{n}\right) \\
& \left.=\mathbf{B} M_{n}, B\right) \times F M_{0},
\end{aligned}
$$

where the coproduct is taken over all ordered lists, $M_{0}, \ldots, M_{n}$. Thus each $n$ simplex of $L_{K} F(C)$ consists of a chain of maps $f_{1}, \ldots, f_{n}$ in $\mathbf{A}$, a map $f: \operatorname{codomain}\left(f_{n}\right)$ $\rightarrow B$ in $\mathbf{B}$ and an element of $F M_{0}$. The isomorphism is thus obvious.

This proposition allows an easy proof to be given of the fact that the set of connected components, $\pi_{0}(\operatorname{Ner}(\tau(B, F)))$, is the Kan extension.

Proposition 6.5. There is a natural isomorphism

$$
\pi_{0} L_{K} F \cong \operatorname{Lan}_{K} F \text {. }
$$

Proof. Recall that we have abused notation in writing $F: \mathbf{A} \rightarrow \mathbf{S}_{*}$ for what should have been $K(F, O)$; this will be crucial in what follows.

Suppose $G: \mathbf{B} \rightarrow$ Set $_{*}$; then

$$
\begin{aligned}
\operatorname{Sets}_{*}^{\mathbf{B}}\left(\pi_{0} L_{K} F, G\right) & \cong S_{*}^{\mathbf{B}}(L F, K(G, O)) \\
& \cong S_{*}^{\mathbf{A}}(K(F, O), K(G K, O)) \\
& \cong \operatorname{Set}_{*}^{\mathbf{A}}(F, G K) \\
& \cong \operatorname{Sets}_{*}^{\mathbf{B}}\left(\operatorname{Lan}_{K} F, G\right),
\end{aligned}
$$

as expected.

This proposition not only suggests a potential link in general between coherent extensions and derived functors, but helps the interpretation of the coherent extensions in a special case.

The usefulness of the general formulation of coherent extension as an extension of the derived functor formulation and also as a potential foundation for abstract homotopy theories, as suggested by Heller and Grothendieck, will depend on our ability to mimic for the coherent situation the principal results and methods of ordinary category theory. One such, the Yoneda lemma formulation, has already been studied; another, the Godement interchange law, will be looked at in the next section.

\section{The coherent Godement LaW}

Given functors $M, N: \mathbf{A} \rightarrow \mathbf{B}, F, G: \mathbf{B} \rightarrow \mathbf{C}$, there are two obvious ways to define a "composition"

$$
\operatorname{Nat}(M, N) \times \operatorname{Nat}(F, G) \rightarrow \operatorname{Nat}(F M, G N) .
$$

The two composites send $(\alpha, \beta)$ to $\beta N \circ F \alpha$ and to $G \alpha \circ \beta M$ respectively and it is an elementary exercise to prove these are equal.

Although elementary, this interchange law,

$$
\beta N \circ F \alpha=G \alpha \circ \beta M,
$$


is fundamental to the development of the calculus of natural transformations and thus to category theory. The corresponding law for coherent transformations is equally important, but is not elementary to prove.

Suppose that $\mathbf{A}, \mathbf{B}, \mathbf{C}$ are $S$-categories, with $\mathbf{C}$ locally weakly Kan, and that $M, N: \mathbf{A} \rightarrow \mathbf{B}, F, G: \mathbf{B} \rightarrow \mathbf{C}$ are $S$-functors. Then we have two simplicial maps

$$
\operatorname{Coh}(M, N) \times \operatorname{Coh}(F, G) \longrightarrow \operatorname{Coh}(F M, G N)
$$

defined up to homotopy. These use the composition studied in section 4 , hence their definition only up to homotopy.

The general "yoga" of homotopy coherence should suggest that these two composites,

$$
\operatorname{Coh}(M, N) \times \operatorname{Coh}(F, G) \rightarrow \operatorname{Coh}(F M, G N),
$$

should be homotopic by a constructible homotopy. Again the experience of handling composition in section 4 suggests that there might hopefully be some "subdivision" of $\Delta \times \Delta[1]$ with a fairly naturally defined map from $\operatorname{Coh}(M, N) \times \operatorname{Coh}(F, G)$ to

$$
\operatorname{hom}(\operatorname{Subdivision,~} Y(F M, G N)) \text {, }
$$

and then a filling argument to get from there to

$$
\operatorname{hom}(\Delta, Y(F M, G N))=\operatorname{Coh}(F M, G N) .
$$

This is the plan we follow, but first we note:

Lemma 7.1. There is a natural isomorphism

$$
\Delta[n] \times \Delta[1]=\int^{[p],[q]} \Delta([p]+[q],[n]) \times \Delta([p]+[q]) .
$$

Proof. (Although this is fairly obvious, the insight gained here will be used later on and so we provide a proof.)

We first note that

$$
(\Delta[n] \times \Delta[1])_{r}=\Delta([r],[n]) \times \Delta([r],[1]) .
$$

Any surjective map, $\sigma$, from $[r]$ to $[1]$ partitions $[r]$ as $[p]+[q]$ where $\sigma([p])=\{0\}$, $\sigma([q])=\{1\}$. The top dimensional simplices of $\Delta[n] \times \Delta[1]$ are of dimension $n+1$ and correspond exactly to those $p, q$ with $p+q=n$.

(One can interpret $\Delta([p]+[q])$ as $\Delta[p] * \Delta[q]$, that is, the join of the two simplices. In this way the lemma reduces to an interpretation of well known combinatorial features within the formation of a coend.)

We will write $D(p, q ; n)$ for the set of pairs $(f, g)$ where $f \in \Delta([p]+[q],[n])$, $g \in \Delta([p]+[q],[1])$ such that $g(i)=0$ if $i \leq p, g(i)=1$ if $i>p$. In this notation we have

$$
\Delta[n] \times \Delta[1]=\int^{[p],[q]} D(p, q ; n) \times \Delta([p]+[q]) .
$$

We return next to the given data $M, N: \mathbf{A} \rightarrow \mathbf{B}, F, G: \mathbf{B} \rightarrow \mathbf{C}$, and assume we are given $A_{0}, \ldots, A_{n}$. With this we can pick $p, q, r$ such that $p+q+r=n$ and then try to make the transition between the two hopefully homotopic maps bit by bit. 
We suppose that we have all the coherence data encoded in the cosimplicial simplicial sets $Y(M, N)$ and $Y(F, G)$. From $A_{p}, \ldots, A_{p+q}$, we obtain (from $Y(M, N)$ ) a map:

$$
\mathbf{A}\left(A_{p}, A_{p+1}\right) \times \ldots \times \mathbf{A}\left(A_{p+q-1}, A_{p+q}\right) \times \Delta[q] \rightarrow \mathbf{B}\left(M A_{p}, N A_{p+q}\right) .
$$

From Lemma 7.1, we have maps

$$
\mathbf{A}\left(A_{0}, A_{1}\right) \times \ldots \times \mathbf{A}\left(A_{p-1}, A_{p}\right) \rightarrow \mathbf{B}\left(M A_{0}, M A_{1}\right) \times \ldots \times \mathbf{B}\left(M A_{p-1}, M A_{p}\right),
$$

and (for $p+q+r=n$ ),

$$
\begin{aligned}
\mathbf{A}\left(A_{p+q}, A_{p+q+1}\right) \times \ldots \times \mathbf{A} & \left(A_{n-1}, A_{n}\right) \\
& \rightarrow \mathbf{B}\left(N A_{p+q}, N A_{p+q+1}\right) \times \ldots \times \mathbf{B}\left(N A_{n-1}, N A_{n}\right),
\end{aligned}
$$

which combine to give a map

$$
\mathbf{A}\left(A_{0}, A_{1}\right) \times \ldots \times \mathbf{A}\left(A_{n-1}, A_{n}\right) \times \Delta[q] \rightarrow \mathbf{B}\left(M A_{0},{ }_{-}\right) \times \ldots \times \mathbf{B}\left({ }_{-}, N A_{n}\right) .
$$

(Note this construction may jump to $N$ immediately or at the last moment or somewhere in between; however it will always end up at an NA and start at an MA.) Thus the codomain of this map has $p+r+1$ terms in it and within $Y(F, G)$, we have the data indexed by $M A_{0}, \ldots, M A_{p}, N A_{p+q}, \ldots, N A_{n}$. Combining this gives us elements of the form

$$
\mathbf{A}\left(A_{0}, A_{1}\right) \times \ldots \times \mathbf{A}\left(A_{n-1}, A_{n}\right) \times \Delta[q] \times \Delta([p]+[r]) \rightarrow \mathbf{C}\left(F M A_{0}, G N A_{n}\right),
$$

which in the two cases $p=0$ and $r=0$ correspond to the two maps defined earlier.

We are now, as hoped, in a similar position to our earlier one involving the $S d \Delta[n]$ and $\operatorname{hom}(S d \Delta, Y(F, H))$. We denote by $D(n)$ the simplicial set

$$
\int^{[p],[q],[r]} D(p, q, r ; n) \times \Delta([p]+[r]) \times \Delta[q]
$$

where $D(p, q, r ; n)=\{(f, g): f \in \Delta([p]+[q]+[r],[n]), g \in \Delta([p]+[r],[1])$ such that $g(i)=0$ if $i \leq p, g(i)=1$ if $i>p\}$. The above discussion can be summarised as follows:

Lemma 7.2. There is a natural map

$$
\operatorname{Coh}(M, N) \times \operatorname{Coh}(F, G) \rightarrow \operatorname{hom}(D, Y(F M, G N)) .
$$

The points we have not proved explicitly are the variance of $D(n)$ with $n$, the way in which the $d_{p}$ on the first $p$ are compatible with the $d_{0}$ on the $q$-terms, etc. Each of these is routine and so is left out.

We are now in a position to state the main coherent interchange theorem

Theorem 7.3. Let $\mathbf{C}$ be a locally weakly Kan category and let $M, N: \mathbf{A} \rightarrow \mathbf{B}$, $F, G: \mathbf{B} \rightarrow \mathbf{C}$ be $S$-functors. Given a choice of filling scheme for $\operatorname{Subdiag}(\Delta \times \Delta)$ (as outlined in section 4), there is a homotopy

$$
\operatorname{Coh}(M, N) \times \operatorname{Coh}(F, G) \rightarrow \operatorname{Coh}(F M, G N)^{\Delta[1]},
$$

between the two evident composites from

$$
\operatorname{Coh}(M, N) \times \operatorname{Coh}(F, G)
$$

to

$$
\operatorname{Coh}(F M, G N) .
$$


The proof can be reduced, using 7.2 , to proving that there is a map from

$$
\operatorname{hom}(D, Y(F M, G N))
$$

to

$$
\operatorname{hom}(\Delta \times \Delta[1], Y(F M, G N))
$$

that restricts to the filling scheme map on the two ends of the cylinder. (Recall we are thinking of $D(n)$ as being a "subdivision" of $\Delta[n] \times \Delta[1]$, the two ends corresponding to $p=0$ and $r=0$ respectively.)

Lemma 7.4. There is an embedding

$$
D \rightarrow \operatorname{Subdiag}(\Delta \times \Delta) \times \Delta[1]
$$

extending the embedding of $S d \Delta$ on the two ends.

Proof. Recall

$$
D(n)=\int^{[p],[q],[r]} D(p, q, r ; n) \times \Delta([p]+[r]) \times \Delta[q] .
$$

where $(f, g) \in D(p, q, r ; n)$ if $f:[p]+[q]+[r] \rightarrow[n]$ and $g:[p]+[r] \rightarrow[1]$ is such that $g(i)=0$ if $i \leq p, g(i)=1$ if $i>p$; i.e. $g$ is a map from $[p+r+1]$ onto [1] corresponding to the division of $[p+r+1]$ as $[p]+[r]$.

This pair $(f, g)$ defines a map

$$
(f, g): \Delta([p]+[r]) \rightarrow \Delta[n] \times \Delta[1]
$$

by

$$
(f, g)(i)= \begin{cases}(f(i), 0) & \text { if } i \leq p \\ (f(q+i), 1) & \text { if } i>p\end{cases}
$$

whilst $f$ restricted to $[q]$ defines a map from $\Delta[q]$ into $\Delta[n]$. The checking that this defines an embedding as claimed resembles that giving the embedding of $S d \Delta$ into $\operatorname{Subdiag}(\Delta \times \Delta)$ and will be omitted.

We had a decomposition of $\Delta[n] \times \Delta[1]$ into its constituent $(n+1)$-simplices, labelled by the surjections from $[n+1]$ to [1] and hence by decompositions of $[n+1]$ as $[p]+[r]$. For each triple $p, q, r$ as in the above, we have a certain class of decompositions $[s]+[t]$ of $p+q+r$ where $s \geq p$ and $t \geq r$; i.e. the concatenation point of $[s]+[t]$ occurs within the $[q]$ of $[p]+[q]+[r]$. For each such, we obtain a subdivision of $[q]$ as $\left[s_{1}\right]+\left[t_{1}\right]$ and $\left([p]+\left[s_{1}\right]\right)+\left(\left[t_{1}\right]+[r]\right)=[p]+[q]+[r]$.

Lemma 7.5. There is a decomposition of $D(n)$, analogous to that of $\Delta[n] \times \Delta[1]$ in terms of $(n+1)$-simplices, such that corresponding to $[s]+[t]$ we have $S d \Delta[s] *$ $S d \Delta[t]$, the join of the two subdivided complexes.

The proof is merely the verification that for given $[s]$, $[t]$, the set of $[p]+[q]+[r]$ with $p \leq s$ and $r \leq t$ defines the join; however this is essentially what we have checked above.

Corollary 7.6. The geometric realisation $|D(n)|$ is naturally homeomorphic to $|\Delta[n] \times \Delta[1]|$.

Proof. It suffices to point out that $|S d \Delta[s] * S d \Delta[t]| \cong|\Delta[s] * \Delta[t]|$.

Corollary 7.7. The coherent interchange law holds when $\mathbf{C}$ is a locally Kan category. 
Proof. As in the case that $Y(F M, G N)$ is a fibrant cosimplicial simplicial set, we can replace each level by Sing $|Y(F M, G N)|$ and obtain a homotopy equivalence

$$
\operatorname{hom}(D, Y(F M, G N)) \rightarrow \operatorname{hom}(D, \text { Sing } \mid Y(F M, G N \mid),
$$

but the codomain here is canonically isomorphic to

$$
\operatorname{hom}(|D|, \mid Y(F M, G N \mid),
$$

which by Corollary 7.6 is

$$
\operatorname{hom}(|\Delta \times \Delta[1]|,|Y(F M, G N)|) .
$$

Reversing the argument yields a map,

$$
\operatorname{hom}(D, Y(F M, G N)) \rightarrow \operatorname{hom}(\Delta, Y(F M, G N)),
$$

with the desired properties.

The version of the interchange law with a locally weakly Kan S-category $\mathbf{C}$ requires more care. We first note that a filling scheme for $S d \Delta$ gives fillers for $S d \Delta[s] * S d \Delta[t]$ within $\operatorname{Subdiag}(\Delta[s] \times \Delta[s]) * \operatorname{Subdiag}(\Delta[t] \times \Delta[t])$ for each $s, t$. The final stage is then to note the decomposition (7.1) of $\Delta[n] \times \Delta[1]$ :

$$
\Delta[n] \times \Delta[1]=\int^{[s],[t]} D(s, t ; n) \times \Delta([s]+[t]) .
$$

Using the "gluing instructions" for the $\Delta([s]+[t])$, we can extend the filling scheme to extend maps with weak Kan codomain from $D$ to a cosimplicial simplicial set $E$ where

$$
E(n)=\int^{[s],[t]} D(s, t ; n) \times \operatorname{Subdiag}(\Delta[s] \times \Delta[s]) * \operatorname{Subdiag}(\Delta[t] \times \Delta[t]) .
$$

(Note this will not be the subdiagonal of $\Delta[n] \times \Delta[n] \times \Delta[1] \times \Delta[1]$.) As the subdiagonals contain the diagonals, there is a map

$$
\Delta[n] \times \Delta[1] \rightarrow E(n)
$$

natural in $[n]$. We thus obtain

$$
\operatorname{hom}(D, Y) \stackrel{\text { filling }}{\rightarrow} \operatorname{hom}(E, Y) \stackrel{\text { rest }}{\rightarrow} \operatorname{hom}(\Delta \times \Delta[1], Y),
$$

where $Y$ is $Y(F M, G N)$. As the filling map is defined using the filling scheme for $S d \Delta$, the extensions can be chosen compatibly with those assumed to be chosen for the compositions. This completes the proof of the coherent Godement interchange law (7.3).

\section{UNIVERSALity RESUlts In A COHERENT SETTING}

It is natural to use the term 'natural'. We have established, for instance, an isomorphism

$$
\operatorname{Coh}(\mathbf{A}, \mathbf{C})(G K, F) \cong \mathbf{C}^{\mathbf{B}}\left(G, R_{K} F\right)
$$

which is natural in $G$. Here however it would be better to cite the homotopy equivalence (cf. 6.1),

$$
\operatorname{Coh}(\mathbf{A}, \mathbf{C})(G K, F) \simeq \operatorname{Coh}(\mathbf{B}, \mathbf{C})\left(G, R_{K} F\right),
$$

which is again natural in $G$. It does not seem to be easy to prove that this homotopy equivalence determines $R_{K} F$ "up to coherent homotopy equivalence" just by using its naturality with respect to $G$. To get such a desirable result, we need to ask what 
happens when $G$ varies coherently. We introduce this idea only in a simple case but feel that, for a thorough development of coherent category theory, an extension of the concept will be necessary.

We say a family of homotopy equivalences

$$
\operatorname{Coh}(G, F) \underset{g_{G}}{\stackrel{f_{G}}{\rightleftarrows}} \operatorname{Coh}\left(G, F^{\prime}\right)
$$

with homotopies $H_{G}: g_{G} f_{G} \simeq I d, K_{G}: f_{G} g_{G} \simeq I d$, is 1-coherent if for any $G_{0}, G_{1}$, the following diagram in which the horizontal maps are compositions,

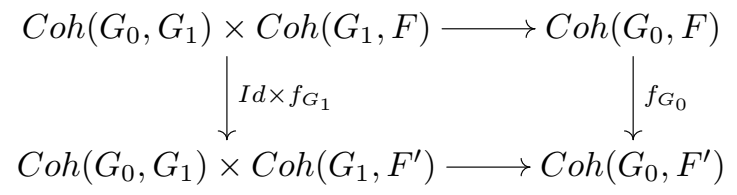

commutes up to a (specified) homotopy.

Remarks. (i) For most of our examples, 1-coherence suffices; however it is the lowest case of a family of " $n$-coherence" conditions. We say a family of homotopy equivalences as above is $n$-coherent if for any $G_{0}, G_{1}, \ldots, G_{n}$, the evident $(n+1)$ cube given by composition made up of a base

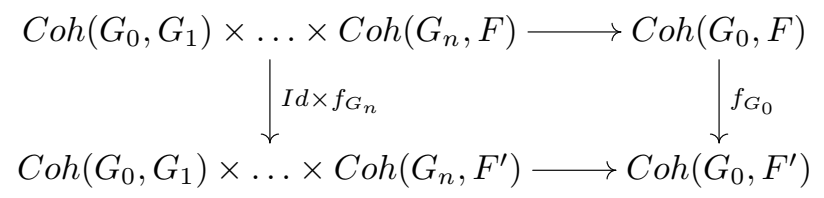

together with the squares coming from partial compositions is a homotopy commutative cube. It is clear (but not proved explicitly) that composition is "coherently as associative as we need", i.e. it is coherently $n$-associative for any $n$, so the above makes sense. We say it is coherent in $G$ if it is $n$-coherent in $G$ for all n.

(ii) The adaption needed to say that the homotopy equivalences

$$
\operatorname{Coh}(G K, F) \simeq \operatorname{Coh}\left(G, R_{K} F\right)
$$

are $n$-coherent in $G$ is minor and will be omitted.

We next point out the 'obvious' meaning of saying that $F$ and $F^{\prime}$ are (coherently) homotopy equivalent. This clearly should mean that there are vertices $f \in$ $\operatorname{Coh}\left(F, F^{\prime}\right)_{0}, g \in \operatorname{Coh}\left(F^{\prime}, F\right)_{0}$ and 1-simplices $H \in \operatorname{Coh}(F, F), K \in \operatorname{Coh}\left(F^{\prime}, F^{\prime}\right)$, so that writing $g f$ for $c_{F, F}^{F^{\prime}}(f, g)$, etc., we have

$$
\begin{aligned}
& d_{0} H=g f, \quad d_{1} H=I d_{F}, \\
& d_{0} K=f g, \quad d_{1} K=I d_{F^{\prime}} .
\end{aligned}
$$

Provided the ambient category codomain of all these functors is locally weakly Kan, we can build up such an $H$ or $K$ by a composite of 1 -simplices, as we shall need to do shortly.

The best example of a family of homotopy equivalences

$$
\operatorname{Coh}(G, F) \rightarrow \operatorname{Coh}\left(G, F^{\prime}\right)
$$

which is coherent in $G$, as above, comes from composition along a coherent homotopy equivalence. Before stating and proving a converse, we note the following: 
Proposition 8.1. If $f_{G}: \operatorname{Coh}(G, F) \rightarrow \operatorname{Coh}\left(G, F^{\prime}\right)$ is a family of homotopy equivalences satisfying $n$-coherence in $G$, then provided the ambient category is locally Kan, for any choices $g_{G}: \operatorname{Coh}\left(G, F^{\prime}\right) \rightarrow \operatorname{Coh}(G, F)$ of homotopy inverses for $f_{G}$, the family $\left\{g_{G}\right\}$ also satisfies n-coherence in $G$.

Proof. This is a fairly routine application of Theorem 1.1 of our paper [21] applied to the $n$-cube of partial compositions following from that result by considering $f$ as the homotopy coherent mapping from the $n$-cube corresponding to $F$ to that corresponding to $F^{\prime}$.

This proposition thus points out that the lack of symmetry in the definition of "1-coherent in $G$ " is not really there.

Theorem 8.2 (Universality up to coherent homotopy equivalences). Let $\mathbf{A}, \mathbf{B}$ be $S$-categories with $\mathbf{B}$ locally Kan and let $F, F^{\prime}, \mathbf{A} \rightarrow \mathbf{B}$ be $S$-functors. Suppose there is a family of homotopy equivalences

$$
\left\{f_{G}: \operatorname{Coh}(G, F) \rightarrow \operatorname{Coh}\left(G, F^{\prime}\right)\right\}
$$

with supplementary data $\left\{g_{G}, H_{G}, K_{G}\right\}$, that is 1-coherent in $G$; then there is a coherent homotopy equivalence

$$
f: F \rightarrow F^{\prime}
$$

and $f_{G}$ is homotopic to the family induced by $f$.

Proof. Taking $G=F$ gives $f_{F}$ and we set $f=f_{F}\left(I d_{F}\right)$. Similarly we have $g_{F^{\prime}}\left(I d_{F^{\prime}}\right)$ and set it equal to $g$. Thus $f: F \rightarrow F^{\prime}, g: F^{\prime} \rightarrow F$.

Since $f$ is 1-coherent we have a diagram

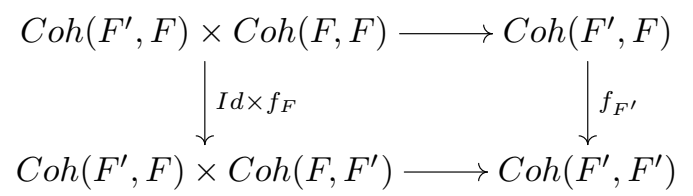

and a homotopy between the composites.

We follow around the pair of 0 -simplices corresponding to $\left(g, I d_{F}\right)$. Since $I d_{F}$ is natural, the composite of these two will be $g$ itself, so around the square clockwise gives us

$$
f_{F^{\prime}}(g)=f_{F^{\prime}} g_{F^{\prime}}\left(I d_{F^{\prime}}\right) \simeq I d_{F^{\prime}}
$$

by the homotopy $K_{F^{\prime}}$. Going around counterclockwise gives the (chosen) composite $f g$. The homotopy in the square restricted to $\left\{\left(g, I d_{F}\right)\right\}$ gives a 1-simplex joining $f g$ and $f_{F^{\prime}}(g)$ and as $\operatorname{Coh}\left(F, F^{\prime}\right)$ is a Kan complex, we obtain a 1-simplex joining $f g$ to the identity on $F^{\prime}$.

Using 8.1 we next reverse the rôles of $f$ and $g$ to obtain the first part of the result.

For the second part we use 1-coherence in $G$ in the general case giving

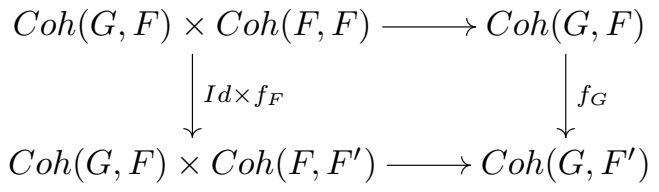

Now restrict to $\operatorname{Coh}(G, F) \times\left\{I d_{F}\right\}$ in the top left corner. 
The top horizontal map is, by assumption (see Proposition 4.5), the identity whilst the counterclockwise path yields post-composition with $f$. Thus $f_{G}$ is homotopic to the map induced by postcomposition with $f$ as claimed.

This result allows us to "tidy up" the ideas introduced in sections 5 and 6 about coherent extensions. The "obvious" coherent version of the definition of a (right) coherent Kan extension would now be:

Let $K: \mathbf{A} \rightarrow \mathbf{B}, F: \mathbf{A} \rightarrow \mathbf{C}$ be $S$-functors; then a functor $R: \mathbf{B} \rightarrow \mathbf{C}$ is a coherent (right) Kan extension of $F$ along $K$ if there is a family of homotopy equivalences

$$
\operatorname{Coh}(G K, F) \rightarrow \operatorname{Coh}(G, R)
$$

that are coherent in $G: \mathbf{B} \rightarrow \mathbf{C}$.

We can draw several immediate consequences of this, given the results of section 6.

Corollary 8.3. (i) If $\mathbf{C}$ is a locally Kan $S$-category in the above definition, then $R$ is determined up to coherent homotopy equivalence.

(ii) If $\mathbf{C}$ is a locally Kan and complete, cotensored $S$-category, then any coherent right Kan extension $R$ (as above) is coherently homotopy equivalence to $R_{K} F$.

Proof. (i) is immediate; (ii) needs a verification that the homotopy equivalences of 6.1 are (at least) 1-coherent, but that is clear.

Remarks. (i) It would be pleasing and useful to be able to weaken 'locally Kan' in the above to 'locally weakly Kan' as this would allow an interpretation in terms of $\infty$-categories.

(ii) There is an obvious similarity between the above definition of "coherent in $G$ " and our earlier discussion of morphisms $\{f(A)\}$ that vary coherently in $A$ to give a coherent map $f \in \operatorname{Coh}(F, G)$. The main difference is that for the version needed here, the specification of a single higher homotopy will not suffice. Even for 2 -coherence, the result is a homotopy coherent cube or prism rather than a single 2simplex. This complication occurs because the composition and higher associativity of the information in $\mathbf{A}$ is exact, whilst in the " $H$-category" $\operatorname{Coh}(\mathbf{A}, \mathbf{B})$, these are coherent only. We still can do much but it takes more care! This suggests that a theory of $H$-simplicial categories might be developed in which one had $H$ simplicial functors etc., in such a way that the two concepts were special cases of a wider concept. The 'pay-off' of such a conjectured theory is not yet clear and the technical difficulties that might be encountered have not been analysed.

\section{EVALUATION OF COHERENT MORPHISMS}

Given two bifunctors, $S, T: \mathbf{A}^{o p} \times \mathbf{A} \rightarrow \mathbf{S}$ there is an "evaluation map":

$$
\operatorname{Nat}(S, T) \times \int_{A} S(A, A) \rightarrow \int_{A} T(A, A)
$$

by the universal property of ends. Here we use Nat as a shorthand for the simplicial set of S-natural transformations.

There is a coherent version of this, but as one might expect, the map is only defined up to homotopy as it is given by a choice of filler and, of course, $T$ must take 'Kan' values. 
Proposition 9.1. Let $S, T: \mathbf{A}^{o p} \times \mathbf{A} \rightarrow \mathbf{S}$ be $S$-functors so that $T\left(A, A^{\prime}\right)$ is always a Kan complex. Then there is a map

$$
\operatorname{Coh}(S, T) \times \oint_{A} S(A, A) \rightarrow \oint_{A} T(A, A),
$$

defined uniquely up to homotopy, induced by evaluation of coherent transformations at the various indices.

Proof. Collecting up the various defining formulae:

(i) $\operatorname{Coh}(S, T)=\int \mathbf{S}\left(\left(\widehat{\mathbf{A}^{o p} \times \mathbf{A}}\right)\left(\left(A, A^{\prime}\right),\left(B, B^{\prime}\right)\right), \mathbf{S}\left(S\left(A, A^{\prime}\right), T\left(B, B^{\prime}\right)\right)\right)$ where the end is taken over all pairs $\left(A, A^{\prime}\right)$ and $\left(B, B^{\prime}\right)$ in $\mathbf{A}^{o p} \times \mathbf{A}$ and

(ii) $\oint_{A} S(A, A)=\int_{C, C^{\prime}} \mathbf{S}\left(\hat{\mathbf{A}}\left(C, C^{\prime}\right), S\left(C, C^{\prime}\right)\right)$.

Together these give us a map from $\operatorname{Coh}(S, T) \times \oint_{A} S(A, A)$ to

$$
\int \mathbf{S}\left(\left(\widehat{\mathbf{A}^{o p} \times \mathbf{A}}\right)\left(\left(A, A^{\prime}\right),\left(B, B^{\prime}\right)\right) \times \hat{\mathbf{A}}\left(C, C^{\prime}\right), \mathbf{S}\left(S\left(A, A^{\prime}\right), T\left(B, B^{\prime}\right)\right) \times S\left(C, C^{\prime}\right)\right)
$$

where the end is taken over all triples, $\left(A, A^{\prime}\right),\left(B, B^{\prime}\right),\left(C, C^{\prime}\right)$ in $\mathbf{A}^{o p} \times \mathbf{A}$. Hence by restriction of the indexing category for the end together with use of the natural maps:

$$
\mathbf{S}\left(S\left(A, A^{\prime}\right), T\left(B, B^{\prime}\right)\right) \times S\left(A, A^{\prime}\right) \rightarrow T\left(B, B^{\prime}\right),
$$

we get a map from $\operatorname{Coh}(S, T) \times \oint_{A} S(A, A)$ to

$$
\int_{\left(A, A^{\prime}\right),\left(B, B^{\prime}\right)} \mathbf{S}\left(\left(\widehat{\mathbf{A}^{o p} \times \mathbf{A}}\right)\left(\left(A, A^{\prime}\right),\left(B, B^{\prime}\right)\right) \times \hat{\mathbf{A}}\left(A, A^{\prime}\right), T\left(B, B^{\prime}\right)\right),
$$

which is isomorphic to

$$
\int_{B, B^{\prime}} \mathbf{S}\left(\int^{\left(A, A^{\prime}\right)}\left(\widehat{\mathbf{A}^{o p} \times \mathbf{A}}\right)\left(\left(A, A^{\prime}\right),\left(B, B^{\prime}\right)\right) \times \hat{\mathbf{A}}\left(A, A^{\prime}\right), T\left(B, B^{\prime}\right)\right) .
$$

Our aim is to get to $\int_{B, B^{\prime}} \mathbf{S}\left(\hat{\mathbf{A}}\left(B, B^{\prime}\right), T\left(B, B^{\prime}\right)\right)$; hence we extract the term in the coend part of the expression:

(*)

$$
\begin{aligned}
\int^{\left(A, A^{\prime}\right)} & \left(\widehat{\mathbf{A}^{o p} \times \mathbf{A}}\right)\left(\left(A, A^{\prime}\right),\left(B, B^{\prime}\right)\right) \times \hat{\mathbf{A}}\left(A, A^{\prime}\right) \\
= & \int^{\left(A, A^{\prime}\right)} \int^{[n],[m]} X_{\mathbf{A}^{o p} \times \mathbf{A}}\left(\left(A, A^{\prime}\right),\left(B, B^{\prime}\right)\right)_{n,-} \times \Delta[n] \times A\left(A, A^{\prime}\right)_{m,-} \times \Delta[m] .
\end{aligned}
$$

This gives as 'integrand'

$$
\begin{aligned}
\coprod_{\mathcal{I}_{n, m}}\left(\mathbf{A}^{o p} \times \mathbf{A}\right)\left(\left(A, A^{\prime}\right),\left(A_{0}, A_{0}^{\prime}\right)\right) & \times \ldots \times\left(\mathbf{A}^{o p} \times \mathbf{A}\right)\left(\left(A_{n}, A_{n}^{\prime}\right),\left(B, B^{\prime}\right)\right) \\
& \times \Delta[n] \times \mathbf{A}\left(A, C_{0}\right) \times \ldots \times \mathbf{A}\left(C_{m}, A^{\prime}\right) \times \Delta[m]
\end{aligned}
$$

where $\mathcal{I}_{n, m}=\left\{A_{0}, \ldots, A_{n}, A_{0}^{\prime}, \ldots, A_{n}^{\prime}, C_{0}, \ldots C_{m}\right\}$ and since

$$
\left(\mathbf{A}^{o p} \times \mathbf{A}\right)((W, X),(Y, Z))=\mathbf{A}(Y, W) \times \mathbf{A}(X, Z),
$$


this can be rewritten as

$$
\begin{aligned}
\coprod_{\mathcal{I}_{n, m}} \mathbf{A}\left(B, A_{n}\right) \times \ldots \times \mathbf{A}\left(A_{0}, A\right) & \times \mathbf{A}\left(A, C_{0}\right) \times \ldots \times \mathbf{A}\left(C_{m}, A^{\prime}\right) \\
& \times \mathbf{A}\left(A^{\prime}, A_{0}^{\prime}\right) \times \ldots \times \mathbf{A}\left(A_{n}^{\prime}, B^{\prime}\right) \times \Delta[n] \times \Delta[m] .
\end{aligned}
$$

Now "integrating" over A and $A^{\prime}$, we get that the coend (*) above reduces by 'Fubini' to

$$
\int^{[m],[n]} \Delta\left([n]^{o p}+[m]+[n],[s]\right) \times \Delta[n] \times \Delta[m] \times X\left(B, B^{\prime}\right)_{s},
$$

somewhat as in our discussion of composition in section 4 . Thus we have shown that the evaluation maps induce a natural map

$$
\operatorname{Coh}(S, T) \times \oint S(A, A) \rightarrow \operatorname{hom}(E, Y(T))
$$

where $E$ is the cosimplicial simplicial set given by

$$
E(s)=\int^{[m],[n]} \Delta\left([n]^{o p}+[m]+[n],[s]\right) \times \Delta[n] \times \Delta[m] .
$$

Each "block" of maximal dimension, $s$, in this, i.e. each $\Delta[n] \times \Delta[m]$ with $m+n=s$,

\begin{tabular}{|c|c|c|c|c|c|c|c|c|c|c|c|c|c|}
\hline domain & 2 & $<$ & 1 & $<$ & 0 & $<$ & $0^{\prime}$ & $<$ & $0^{\prime \prime}$ & $<$ & $1^{\prime \prime}$ & $<$ & $2^{\prime \prime}$ \\
\hline \multirow{4}{*}{ image } & 0 & $<$ & 1 & $=$ & 1 & $=$ & 1 & $=$ & 1 & $<$ & 2 & $=$ & 2 \\
\hline & 0 & $=$ & 0 & $<$ & 1 & $=$ & 1 & $=$ & 1 & $=$ & 1 & $<$ & 2 \\
\hline & 0 & $<$ & 1 & $<$ & 2 & $=$ & 2 & $=$ & 2 & $=$ & 2 & $=$ & 2 \\
\hline & 0 & $=$ & 0 & $=$ & 0 & $=$ & 0 & $=$ & 0 & $<$ & 1 & $<$ & 2 \\
\hline
\end{tabular}
can be assigned a labelling map with domain $[n]^{o p}+[m]+[n]$ with the characteristics: restricted to the central $[m]$, it is injective, whilst on the side it repeats values at different positions in the two copies of $[n]$. For example, for $s=2$ with $m=0$, $n=2$, we have labels

similarly for $m=1, n=1$ and $m=2, n=0$, again for $s=2$. Any other labelling map can be reduced to a face or degeneracy of one of these. The combinatorial structure of these simplicial sets is similar to that of the triple subdivision of $\Delta$ discussed briefly when we discussed the coherent associativity of compositions. The only difference is the reverse direction on the first sector. For fixed $s$ and $m$, take $n_{1}, n_{2}$ such that $n_{1}+n_{2}=n=s-m$; then $\Delta\left[n_{1}\right] \times \Delta\left[n_{2}\right]$ is given by all shuffles of $n_{1}$ through $n_{2}$, hence by the distinct positions at which repeats can occur in the two copies of $n$ in the expression for $E(s)$, so apart from the reversal of the order, $E(s)$ is "essentially" the same as

$$
\int^{[m],\left[n_{1}\right],\left[n_{2}\right]} \Delta\left(\left[n_{1}\right]+[m]+\left[n_{2}\right]\right) \times \Delta\left[n_{1}\right] \times \Delta[m] \times \Delta\left[n_{2}\right],
$$

i.e. the triple subdivision of $\Delta[s]$.

By "essentially the same", we mean that

$$
|E(s)| \cong|\Delta[s]| \cong \Delta^{s}
$$

so provided $T$ takes Kan values, we can construct a map

$$
\operatorname{hom}(E, Y(T)) \rightarrow \operatorname{hom}(\Delta, Y(T))
$$


by choosing an inverse for the natural homotopy equivalence between $Y(T)$ and $\operatorname{Sing}(|Y(T)|)$, then using adjunctions to give

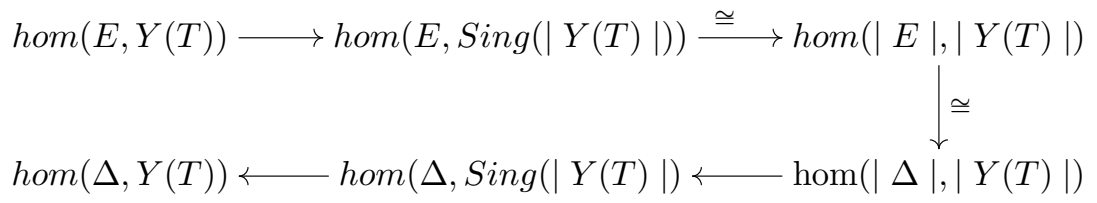

This completes the proof.

Corollary 9.2. Let $S: \mathbf{A}^{o p} \times \mathbf{A} \rightarrow \mathbf{S}, T: \mathbf{A}^{o p} \times \mathbf{A} \rightarrow \mathbf{B}$ be $\mathbf{S}$-bifunctors such that $T\left(A, A^{\prime}\right)$ is always fibrant and suppose $\mathbf{B}$ is cotensored. Then evaluation induces a map

$$
\bar{B}(S, T) \rightarrow \bar{B}(\oint S, \oint T)
$$

Proof. First note that, when $\mathbf{B}=\mathbf{S}$, this is a restatement of 9.1. To reduce it to 9.1 in general, suppose $B$ is an object of $\mathbf{B}$ and apply 9.1 to the functor $\mathbf{B}\left(B, T\left(_{-},{ }_{-}\right)\right)$ in place of $T$. This, after manipulation using the various adjunctions, gives a map

$$
\mathbf{B}(B, \bar{B}(S, T)) \rightarrow \mathbf{B}(B, \bar{B}(\oint S, \oint T))
$$

induced by evaluation. This map is natural in $B$.

Finally put $B=\bar{B}(S, T)$ and look at the image of the identity map.

Proposition 9.3 (Evaluation is compatible with composition). If $S, T_{0}, T_{1}$ : $\mathbf{A}^{o p} \times \mathbf{A} \rightarrow \mathbf{S}$ are $S$-bifunctors so that each $T_{i}$ takes Kan values, then there is a homotopy making the square

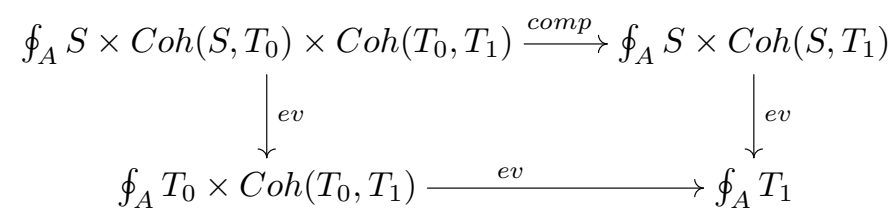

homotopy coherent.

Proof. (This is very similar to the higher associativity results mentioned in section 4.) Evaluating the ends in the top left-hand corner leads once again to a map to an object of the form $\operatorname{hom}\left(X, Y\left(T_{1}\right)\right)$ where $X$ is a cosimplicial simplicial set with realisation $\Delta^{n}$ in codimension $n$. (We leave the precise description of $X$ to the reader.) This as before defines a diagonal for the square. The uniqueness, up to homotopy, of this map then implies that the two composites around the sides of the square are homotopic to it, giving the result.

Remarks. (i) This result, which expresses the 1-coherence of evaluation, has extensions to $n$-coherence and to sequences of bifunctors $T_{i}$ with fibrant values in a cotensored S-category B.

(ii) It seems certain that, like many of our results, a finer proof of this result should exist, i.e. one that provides a sequence of fillers for extending maps defined on $X$ over some subdiagonal and hence the possibility of an extension to the weak Kan situation. Such a proof would seem necessary if one is to escape from the locally Kan case. This case corresponds intuitively to a laxification of a $\infty$-groupoid enriched category whilst the weakly Kan context includes $\infty$-categories. 


\section{Coherent adjunctions}

Following the logic of the previous sections, we next look at the coherent analogue of adjunction. For technical reasons we restrict to locally Kan categories $\mathbf{A}$ and $\mathbf{B}$, and suppose we are given $S$-functors $F: \mathbf{A} \rightarrow \mathbf{B}$ and $G: \mathbf{B} \rightarrow \mathbf{A}$. We will say the functors, $F$ and $G$ are coherent adjoints if there is a coherent homotopy equivalence between $\mathbf{B}\left(F_{-}, *\right)$ and $\mathbf{A}\left({ }_{-}, G *\right)$, where - and $*$ are here used as 'placemarkers', from $\mathbf{A}$ and $*$ from $\mathbf{B}$. Thus we suppose given

$$
\begin{gathered}
f \in \operatorname{Coh}\left(\mathbf{B}\left(F_{-}, *\right), \mathbf{A}\left({ }_{-}, G *\right)\right)_{0}, \\
g \in \operatorname{Coh}\left(\mathbf{A}\left({ }_{-}, G *\right), \mathbf{B}\left(F_{-}, *\right)\right)_{0}, \\
H \in \operatorname{Coh}\left(\mathbf{B}\left(F_{-}, *\right), \mathbf{B}\left(F_{-}, *\right)\right)_{1}, \\
K \in \operatorname{Coh}\left(\mathbf{A}\left({ }_{-}, G *\right), \mathbf{A}\left({ }_{-}, G *\right)\right)_{1}
\end{gathered}
$$

such that $H: g f \simeq I d, K: f g \simeq I d$, assuming a choice of composition has been made. Restricting $f$ along $F$, we get

$$
f_{-, F} \in \operatorname{Coh}\left(\mathbf{B}\left(F_{-}, F_{-}\right), \mathbf{A}\left({ }_{-}, G F_{-}\right)\right)_{0}
$$

and hence by 9.1 , a coherent map

$$
\eta=f_{-, F_{-}}\left(I d_{F}\right) \in \operatorname{Coh}\left(I d_{A}, G F\right)_{0} .
$$

Similarly one gets

$$
\varepsilon=g_{G_{-,-}}\left(I d_{G}\right) \in \operatorname{Coh}\left(F G, I d_{B}\right)_{0}
$$

Proposition 10.1. Suppose $\mathbf{A}, \mathbf{B}$ locally Kan, and $F: \mathbf{A} \rightarrow \mathbf{B}, G: \mathbf{B} \rightarrow \mathbf{A}$ are given $S$-functors. Then for any $S$-functors $H_{0}, H_{1}: \mathbf{B} \rightarrow \mathbf{A}$, the two composite maps:

$$
\begin{aligned}
\operatorname{Coh}\left(H_{1}, H_{0}\right) \times \operatorname{Coh}\left(\mathbf{B}\left(F_{-}, I d_{B}\right), \mathbf{A}\left(I d_{A}, G\right)\right) \times \operatorname{Coh}\left(F H_{0}, I d_{B}\right) \\
\rightarrow \operatorname{Coh}\left(\mathbf{B}\left(F H_{0}, I d_{B}\right), \mathbf{B}\left(F H_{1}, I d_{B}\right)\right) \\
\left.\quad \times \operatorname{Coh}\left(\mathbf{B}\left(F H, I d_{B}\right), \mathbf{A}\left(H_{1}, G\right)\right) \times \operatorname{Coh}\left(F H_{0}, I d_{B}\right)\right) \\
\stackrel{\operatorname{comp}}{\rightarrow} \operatorname{Coh}\left(\mathbf{B}\left(F H_{0}, I d_{B}\right), \mathbf{A}\left(H_{1}, G\right)\right) \times \operatorname{Coh}\left(F H_{0}, I d_{B}\right) \\
\stackrel{\text { eval }}{\rightarrow} \operatorname{Coh}\left(H_{1}, G\right)
\end{aligned}
$$

and

$$
\begin{aligned}
& \operatorname{Coh}\left(H_{1}, H_{0}\right) \times \operatorname{Coh}\left(\mathbf{B}\left(F_{-}, I d_{B}\right), \mathbf{A}\left(I d_{A}, G\right)\right) \times \operatorname{Coh}\left(F H_{0}, I d_{B}\right) \\
& \rightarrow \operatorname{Coh}\left(\mathbf{B}\left(F H_{0}, I d_{B}\right), \mathbf{A}\left(H_{0}, G\right)\right) \times \operatorname{Coh}\left(\mathbf{A}\left(H_{0}, G\right), \mathbf{A}\left(H_{1}, G\right)\right) \\
& \times \operatorname{Coh}\left(F H_{0}, I d_{B}\right) \\
& \stackrel{\text { eval }}{\longrightarrow} \operatorname{Coh}\left(H_{0}, G\right) \times \operatorname{Coh}\left(\mathbf{A}\left(H_{0}, G\right), \mathbf{A}\left(H_{1}, G\right)\right) \\
& \stackrel{\text { eval }}{\longrightarrow} \operatorname{Coh}\left(H_{1}, G\right)
\end{aligned}
$$

are homotopic.

Proof. Ignoring for the moment the factor $\operatorname{Coh}\left(F H_{0}, I d_{B}\right)$, we note that by the coherent form of the Godement interchange law, the composite:

$$
\begin{aligned}
\operatorname{Coh}\left(H_{1}, H_{0}\right) \times \operatorname{Coh}\left(\mathbf{B}\left(F_{-}, I d_{B}\right), \mathbf{A}\left(I d_{A}, G\right)\right) \\
\quad \rightarrow \operatorname{Coh}\left(\mathbf{B}\left(F H_{0}, I d_{B}\right), \mathbf{B}\left(F H_{1}, I d_{B}\right) \times \operatorname{Coh}\left(\mathbf{B}\left(F H_{1}, I d_{B}\right), \mathbf{A}\left(H_{1}, G\right)\right)\right. \\
\quad \stackrel{\operatorname{comp}}{\rightarrow} \operatorname{Coh}\left(\mathbf{B}\left(F H_{0}, I d_{B}\right), \mathbf{A}\left(H_{1}, G\right)\right)
\end{aligned}
$$


is homotopic to the composite

$$
\begin{aligned}
\operatorname{Coh} & \left(H_{1}, H_{0}\right) \times \operatorname{Coh}\left(\mathbf{B}\left(F_{-}, I d_{B}\right), \mathbf{A}\left(I d_{A}, G\right)\right) \\
& \rightarrow \operatorname{Coh}\left(\mathbf{B}\left(F H_{0}, I d_{B}\right), \mathbf{A}\left(H_{0}, G\right)\right) \times \operatorname{Coh}\left(\mathbf{A}\left(H_{0}, G\right), \mathbf{A}\left(H_{1}, G\right)\right) \\
& \rightarrow \operatorname{Coh}\left(\mathbf{B}\left(F H_{0}, I d_{B}\right), \mathbf{A}\left(H_{1}, G\right)\right) .
\end{aligned}
$$

After forming its product with $\operatorname{Coh}\left(F H_{0}, I d_{B}\right)$ and composing with the evaluation map, this latter composite gives a map which is homotopic to the second composite of the statement of the proposition.

Corollary 10.2. If $F$ and $G$ are coherent adjoints with data as given above then for any $H: \mathbf{B} \rightarrow \mathbf{A}$, the family of mappings

$$
f_{* H,_{-}}: \operatorname{Coh}\left(F H, I d_{B}\right) \rightarrow \operatorname{Coh}(H, G)
$$

is 1-coherent in $H$.

Proof. We need to prove there is a homotopy between the two composites along the sides of the square

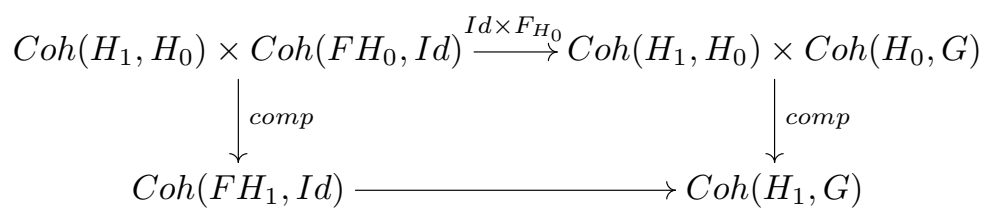

but these two composites are exactly the composite maps of 10.1 restricted, in the second factor, to the 0-simplex $f$.

We note that the method of proof of 10.1 does not depend on the particular forms $\mathbf{B}(F, I d)$ and $\mathbf{A}(I d, G)$; thus we also have variants of $10.1,10.2$ valid for $g$ and the homotopies $H$ and $K$. As a result we obtain:

Corollary 10.3. If $L: \mathbf{B} \rightarrow \mathbf{A}$ is an $S$-functor, then the data

$f_{L}: \operatorname{Coh}\left(F L, I d_{B}\right) \rightarrow \operatorname{Coh}(L, G)$,

$g_{L}: \operatorname{Coh}(L, G) \rightarrow \operatorname{Coh}\left(F L, I d_{B}\right)$,

$H_{L}: \operatorname{Coh}\left(F L, I d_{B}\right) \times \Delta[1] \rightarrow \operatorname{Coh}\left(F L, I d_{B}\right)$,

$K_{L}: \operatorname{Coh}(L, G) \times \Delta[1] \rightarrow \operatorname{Coh}(L, G)$,

(i) vary 1-coherently in $L$,

(ii) satisfy

$$
\begin{aligned}
& H_{L}: g_{L} f_{L} \simeq I d, \\
& K_{L}: f_{L} g_{L} \simeq I d .
\end{aligned}
$$

Thus $f_{L}$ is a 1-coherent homotopy equivalence.

It seems almost certain that, in fact, these data vary coherently in $L$. The proof of this would require certain refinements of earlier results and as only 1-coherence will be needed later, we limit ourselves to this for the moment.

The results we have been using have analogues which prove 1-coherence in the covariant variable. 
Proposition 10.4. Suppose $\mathbf{A}, \mathbf{B}$ locally Kan, and $F: \mathbf{A} \rightarrow \mathbf{B}, G: \mathbf{B} \rightarrow \mathbf{A}$ are given $S$-functors. Then for any $S$-functors $L_{0}, L_{1}: \mathbf{A} \rightarrow \mathbf{B}$, the two composite maps,

$$
\begin{aligned}
\operatorname{Coh} & \left(L_{0}, L_{1}\right) \times \operatorname{Coh}\left(\mathbf{B}\left(F, I d_{B}\right),\left(\mathbf{A}\left(I d_{A}, G\right)\right) \times \operatorname{Coh}\left(F, L_{0}\right)\right. \\
& \rightarrow \operatorname{Coh}\left(\mathbf{B}\left(F, L_{0}\right), \mathbf{B}\left(F, L_{1}\right)\right) \times \operatorname{Coh}\left(\mathbf{B}\left(F, L_{1}\right), \mathbf{A}\left(I d_{A}, G L_{1}\right) \times \operatorname{Coh}\left(F, L_{0}\right)\right. \\
& \stackrel{\text { eval }}{\longrightarrow} \operatorname{Coh}\left(\mathbf{B}\left(F, L_{0}\right), \mathbf{A}\left(I d, G L_{1}\right)\right) \times \operatorname{Coh}\left(F, L_{0}\right) \\
& \stackrel{\text { eval }}{\longrightarrow} \operatorname{Coh}\left(I d_{A}, G L_{1}\right),
\end{aligned}
$$

and

$$
\begin{aligned}
\operatorname{Coh}\left(L_{0}, L_{1}\right) \times \operatorname{Coh}\left(\mathbf{B}\left(F, I d_{B}\right), \mathbf{A}\left(I d_{A}, G\right)\right) \times \operatorname{Coh}\left(F, L_{0}\right) \\
\quad \rightarrow \operatorname{Coh}\left(\mathbf{B}\left(F, L_{0}\right), \mathbf{A}\left(I d_{A}, G L_{0}\right)\right) \\
\quad \times \operatorname{Coh}\left(\mathbf{A}\left(I d_{A}, G L_{1}\right), \mathbf{A}\left(I d_{A}, G L_{1}\right)\right) \times \operatorname{Coh}\left(F, L_{0}\right) \\
\stackrel{\text { eval }}{\longrightarrow} \operatorname{Coh}\left(I d_{A}, G L_{0}\right) \times \operatorname{Coh}\left(\mathbf{A}\left(I d_{A}, G L_{0}\right), \mathbf{A}\left(I d_{A}, G L_{1}\right)\right) \\
\quad \stackrel{\text { eval }}{\longrightarrow} \operatorname{Coh}\left(I d_{A}, G L_{1}\right),
\end{aligned}
$$

are homotopic.

The proof is more or less identical to that of 10.1, so will be omitted.

Corollary 10.5. If $F$ and $G$ are coherent adjoints with data as above then (i) the family of mappings

$$
f_{-, L}: \operatorname{Coh}(F, L) \rightarrow \operatorname{Coh}\left(I d_{A}, G L\right)
$$

is 1-coherent, and

(ii) each $f_{-, L}$ is a 1-coherent homotopy equivalence (as in 10.3).

Of course the two forms, co- and contravariant, of coherence do not interfere with each other, and there is a corresponding bicoherent form of 10.1 and 10.4 unifying the two results. The proof is less neat and as any use of such a result could be reduced to successive uses of 10.1 and 10.3, this result will not be stated here.

Proposition 10.6. Given that $F, G$ are coherent adjoints, there is a homotopy between the two composites of the square

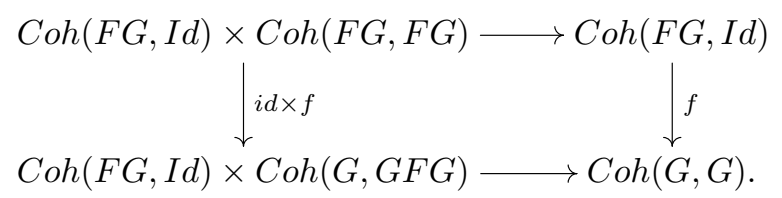

Proof. In 10.4 , replace $\mathbf{B}\left(F, I d_{B}\right)$ by $\mathbf{B}\left(F G, I d_{B}\right)$ and $\mathbf{A}\left(I d_{A}, G\right)$ by $\mathbf{A}(G, G)$. Then taking $L_{0}, L_{1}: \mathbf{B} \rightarrow \mathbf{B}$, the same proof works, giving a homotopy between the two composites from $\operatorname{Coh}\left(L_{0}, L_{1}\right) \times \operatorname{Coh}\left(\mathbf{B}\left(F G, I d_{B}\right), \mathbf{A}(G, G)\right) \times \operatorname{Coh}\left(F G, L_{0}\right)$ to $\operatorname{Coh}\left(G, G L_{1}\right)$. The proof (e.g. in 10.1) of the 1-coherence of $f$ now gives, after slight modification, the homotopy coherence of the square,

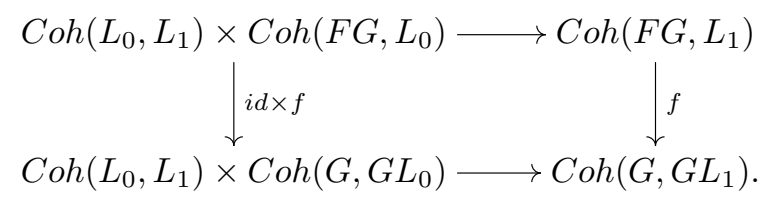


Finally take $L_{0}=F G, L_{1}=I d_{B}$ to get the required result.

Corollary 10.7 (Triangle rule). Given coherent adjoints $F, G$, there is a 1simplex in $\operatorname{Coh}(G, G)$ linking $I d_{G}$ with $G(\varepsilon) \cdot \eta_{G}$.

Proof. (see below in 10.9 and dualise)

We state, without explicit proof, the dual of 10.6.

Proposition 10.8. Given coherent adjoints $F, G$, there is a homotopy between the two composites of the square

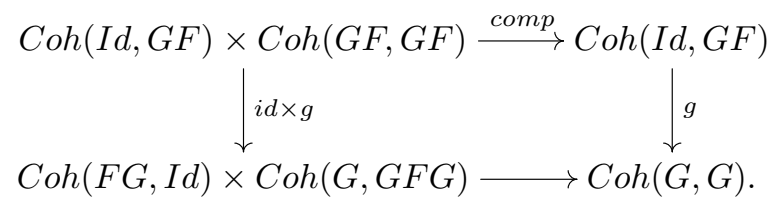

Corollary 10.9 (2nd Triangle rule). Given coherent adjoints $F, G$, there is a 1simplex in $\operatorname{Coh}(F, F)$ linking $I d_{F}$ with $\varepsilon_{F} . F(\eta)$.

Proof. Evaluate the homotopy from 10.8 at the 0 -simplex, $\left\{\eta, I d_{G F}\right\}$. The resulting 1-simplex links $\varepsilon_{F} \cdot F(\eta)$ with $g f\left(I d_{F}\right)$ but $K: g f \simeq I d$, so using the Kan condition to 'compose' 1 -simplices, we get the required 1 -simplex joining $I d_{F}$ and $\varepsilon_{F} \cdot F(\eta)$.

The interpretation of adjoint functors, and even their construction, are aided greatly by the 'universal arrow' type description given for instance in Mac Lane, [38]:

given $F: \mathbf{A} \rightarrow \mathbf{B}, G: \mathbf{B} \rightarrow \mathbf{A}$, ordinary functors between ordinary categories, to say $F$ is left adjoint to $G$ is to say that there is a map

$\eta_{A}: A \rightarrow G F A$ universal for maps into objects of the form $G B$.

This can be conveniently expressed in terms of comma categories or cones. There is an analogous coherent version of this property and as comma $\mathbf{S}$-categories do not seem to be available in the coherent context, we will use instead the notion of homotopy cone (and cocone) introduced by the first author with Bourn in [10].

Given an $S$-functor $F: \mathbf{A} \rightarrow \mathbf{B}$, the homotopy cone on $F$ is a functor

$$
\text { hocone }\left(_{-}, F\right): \mathbf{B}^{o p} \rightarrow \mathbf{S},
$$

given by hocone $(B, F)=\operatorname{Tot} \prod(B, F)$, where $\prod^{\cdot}(B, F)$ is the cosimplicial simplicial set given by

$$
\prod(B, F)^{n}=\prod_{A_{0}, \ldots, A_{n}} \mathbf{S}\left(\mathbf{A}\left(A_{0}, A_{1}\right) \times \ldots \times \mathbf{A}\left(A_{n-1}, A_{n}\right), \mathbf{B}\left(B, F A_{n}\right)\right) ;
$$

i.e. if $c_{B}: \mathbf{A} \rightarrow \mathbf{B}$ is the $S$-functor with constant value $B$, then $\prod^{\bullet}(B, F)=$ $Y\left(c_{B}, F\right)$ and so

$$
\operatorname{hocone}(B, F) \cong \operatorname{Coh}\left(c_{B}, F\right) .
$$

Remark. We point out (cf. [10]) that holimF, if it exists, is a representative of hocone $(,, F)$, i.e.

$$
\mathbf{B}(B, \operatorname{holim} F) \cong \text { hocone }(B, F) .
$$

If $f: B \rightarrow B^{\prime}$ is any morphism in $\mathbf{B}$, we will write $f^{*}:$ hocone $\left(B^{\prime}, F\right) \rightarrow$ hocone $(B, F)$ for the induced map, i.e. $f^{*}=\operatorname{hocone}(f, F)$. 
Dually we have homotopy cocones, hococone $(F, B)$, defined by $C o h\left(F, c_{B}\right)$ and a representative for hococone $\left(F,{ }_{-}\right)$will be a homotopy colimit for $F$.

We apply this terminology to the coherent adjoint situation in the following proposition.

Proposition 10.10. Suppose $F, G$ form a coherent adjoint pair with unit $\eta$ and data $H, K$, etc., as before. Then for any $A$, the identity map on hocone $(A, G)$ factorises up to homotopy as follows:

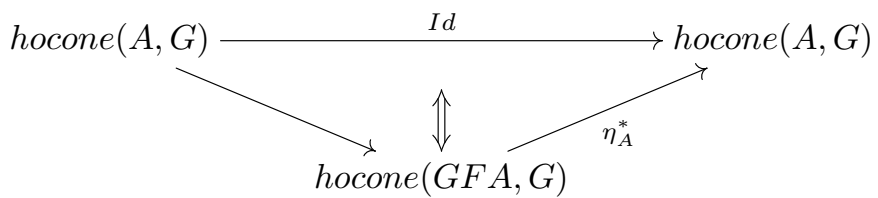

moreover the homotopy varies 1-coherently with A.

Proof. As always we will denote by $c_{A}: \mathbf{B} \rightarrow \mathbf{A}$, the constant $S$-functor with value A, thus $c_{F A}=F c_{A}$ etc. The identification of hocone $(A, G)$ as $C o h\left(c_{A}, G\right)$ means that $g$, the coherent transformation obtained from the initial data by evaluation, induces a map

$$
\operatorname{Coh}\left(c_{A}, G\right) \rightarrow \operatorname{Coh}\left(c_{F A}, I d\right),
$$

which we will denote $g_{A}$.

Proposition 10.4 gives us a homotopy between the two composites in the square (cf. proof of 10.6):

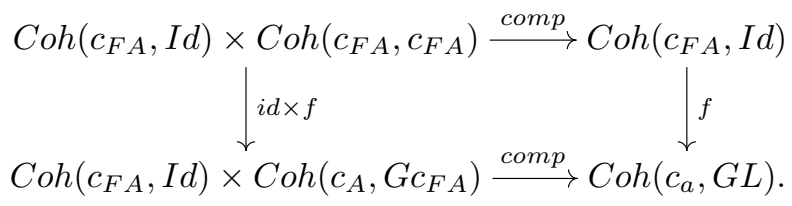

Since $f: \operatorname{Coh}(F, F) \rightarrow \operatorname{Coh}(I d, G F)$ gives $\eta=f\left(I d_{F}\right)$, composition with $c_{A}: \mathbf{B} \rightarrow$ A gives

$$
\eta_{A}=f\left(I d_{F A}\right): \operatorname{Coh}\left(c_{F A}, c_{F A}\right) \rightarrow \operatorname{Coh}\left(c_{A}, G F c_{A}\right),
$$

we can evaluate the above homotopy on the subsimplicial set $C o h\left(c_{F A}, I d\right) \times\left\{I d_{F A}\right\}$ to get a homotopy between $f$ and the composite

$$
\operatorname{Coh}\left(c_{F A}, I d\right) \stackrel{G}{\longrightarrow} \operatorname{Coh}\left(c_{G F A}, G\right) \stackrel{\eta_{A}^{*}}{\longrightarrow} \operatorname{Coh}\left(c_{A}, G\right),
$$

where $G$ denotes the usual mapping associated with composition with the functor $G$. Thus we get

$$
\operatorname{Coh}\left(c_{A}, G\right) \stackrel{G(g)}{\rightarrow} \operatorname{Coh}\left(c_{G F A}, G\right) \stackrel{\eta_{A}^{*}}{\rightarrow} \operatorname{Coh}\left(c_{A}, G\right)
$$

is homotopic to $f . g$ and hence to the identity on $\operatorname{Coh}\left(c_{A}, G\right)$ by evaluation of $K$. The result follows since each of the constructions given is 1-coherent in $A$. (As usual "1-coherent" can probably be replaced by "coherent" here.)

There is clearly a dual to this that we give without separate proof. 
Proposition 10.11. Suppose $F, G$ form a coherent adjoint pair with counit, $\varepsilon$ as above. Then for any $B$, the identity map on hococone $(F, B)$ factorises up to homotopy as follows

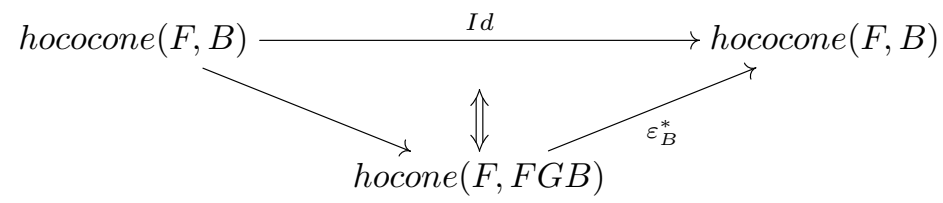

Moreover the homotopy varies 1-coherently in $B$.

Remark. The mapping from hococone $(F, B)$ to hococone $(F, F G B)$ in this diagram is given by the composite

$$
\operatorname{Coh}\left(F, c_{B}\right) \stackrel{f}{\rightarrow} \operatorname{Coh}\left(I d, G c_{B}\right) \stackrel{F}{\rightarrow} \operatorname{Coh}\left(F, F G c_{B}\right) .
$$

\section{REFERENCES}

[1] J. F. Adams, A variant of E.H. Brown's representability theorem, Topology, 10, (1971), 185198. MR 44:1018

[2] D. W. Anderson, Axiomatic Homotopy Theory, in Algebraic Topology, Waterloo 1978, Lecture Notes in Mathematics, No. 741, Springer-Verlag, Berlin, Heidelberg, New York, 1979. MR 81c:55031

[3] M. André, Méthode Simpliciale en Algèbre Homologique et Algèbre Commutative, Lecture Notes in Mathematics, No. 32, Springer-Verlag, Berlin, Heidelberg, New York, (1967). MR 35:5493

[4] M. Artin and B. Mazur, Étale homotopy, Lecture Notes in Mathematics, No. 100, SpringerVerlag, Berlin, Heidelberg, New York, (1969). MR 39:6883

[5] M. Artin and B. Mazur, On the van Kampen Theorem, Topology, 5, (1966), 179-189. MR 33:720

[6] M. Barr and J. Beck, Homology and Standard Constructions, in Seminar on Triples and Categorical Homology Theory, (B. Eckmann, ed.), Lecture Notes in Mathematics, No. 80, pp. 245-335, Springer-Verlag, Berlin, Heidelberg, New York, 1969. MR 41:3562

[7] M. A. Batanin, Coherent categories with respect to monads and coherent prohomotopy theory, Cahiers Top. Géom. Diff. Cat, 34, (1993), 279-304. MR 95b:18006

[8] H. J. Baues, Geometry of loop spaces and the cobar construction, Memoirs Amer. Math. Soc. 25, (1980), Number 230. MR 81 m:55010

[9] H. J. Baues, Algebraic Homotopy, Cambridge Studies in Advanced Mathematics 15, Cambridge Univ. Press, (1989). MR 90i:55016

[10] D. Bourn and J.-M. Cordier, A general formulation of homotopy limits, J. Pure Appl. Algebra, 29, (1983), 129-141. MR 85c:55013

[11] A. K. Bousfield and D. M. Kan, Homotopy Limits, Completions and Localizations, Lecture Notes in Math. No. 304, Springer-Verlag, Berlin, Heidelberg, New York, (1972). MR 51:1825

[12] S. Bozapalides, Théorie formelle des bicatégories, thèse, 3-ème cycle, (1976), Paris. MR 57:16380

[13] S. Bozapalides, Some remarks on Lax presheafs, Illinois J. Math., 24, (1980), 676-680. MR 81m:18006

[14] K. S. Brown, Abstract homotopy theory and generalised sheaf cohomology, Trans. Amer. Math. Soc., 186, (1973), 419-458. MR 49:6220

[15] R. Brown and N. D. Gilbert, Algebraic Models for 3-types and automorphism structures for crossed modules, Proc. London Math. Soc., 59, (1989), 51-73. MR 90e:18015

[16] J.-M. Cordier, Sur la notion de diagramme homotopiquement cohérent, Cahiers Top. et Géom. Diff., 23, (1982), 93-112, Proc., 3ème Coll. sur les Catégories, Amiens, (1980). MR 83g:18018

[17] J.-M. Cordier, Extensions de Kan simplicialement cohérentes, Prépublications, Amiens, (1985).

[18] J.-M. Cordier, Sur les limites homotopiques de diagrammes homotopiquement cohérents, Comp. Math., 62, (1987), 367-388. MR 88m:55029 
[19] J.-M. Cordier, Homologie de Steenrod-Sitnikov et limite homotopique algébrique, Manuscripta Math., 59, (1987), 35-52. MR 88m:55003

[20] J.-M. Cordier, Comparison de deux catégories d'homotopie de morphismes cohérents, Cahiers Top. Géom. Diff. Cat., 33, (1989), 257-275. MR 91d:55005

[21] J.-M. Cordier and T. Porter, Vogt's theorem on categories of homotopy coherent diagrams, Math. Proc. Camb. Phil. Soc., 100, (1986), 65-90. MR 87i:55027

[22] J.-M. Cordier and T. Porter, Coherent Kan Extensions, (i). Simplicially Enriched Ends and Coends, U.C.N.W. Pure Maths. Preprint 86.19, (1986).

[23] J.-M. Cordier and T. Porter, Fibrant diagrams, rectifications and a construction of Loday, J. Pure Appl. Algebra, 67, (1990), 111-124. MR 92a:55017

[24] J.-M. Cordier and T. Porter, Categorical Aspects of Equivariant Homotopy, Applied Categorical Structures (to appear).

[25] W. G. Dwyer and D. M. Kan, Realizing diagrams in the homotopy category by means of diagrams of simplicial sets, Proc. Amer. Math. Soc., 91, (1984), 456-460. MR 86c:55010b

[26] W. G. Dwyer and D. M. Kan, Function complexes for diagrams of simplicial sets, Proc. Kon. Ned. Akad. Wet., 86, (1983), 139-147. MR 85e:55038

[27] D. A. Edwards and H. M. Hastings, Čech and Steenrod Homotopy Theories with Applications to Geometric Topology, Lecture Notes in Mathematics No. 542, Springer-Verlag, Berlin, Heidelberg, New York, (1976). MR 55:1347

[28] A. Elmendorf, Systems of Fixed Point Sets, Trans. Amer. Math. Soc, 277, (1983), 275-284. MR 84f: 57029

[29] P. E. Gardener, Generalized Derived Functors, Thesis, University of Warwick, 1981.

[30] J. M. Gray, Closed categories, lax limits and homotopy limits, J. Pure Appl. Algebra, 19, (1980), 127-158. MR 82f:18007a

[31] A. Grothendieck, (1983), Pursuing Stacks, typed manuscript, (c.600 pages).

[32] B. Günther, The use of semisimplicial complexes in strong shape theory, Glasnik Mat., 27, (1992), 101-144. MR 94g:55014

[33] A. Heller, Homotopy in Functor Categories, Trans. Amer. Math. Soc., 272, (1982), 185-202. MR 84j:55009a

[34] A. Heller, Homotopy Theories, Memoirs Amer. Math. Soc. Vol. 71, No. 383, Amer. Math. Soc., Providence, R.I., (1988). MR 89b:55013

[35] K. H. Kamps and T. Porter, Abstract homotopy and simple homotopy theory, World Scientific, (1996?).

[36] G. M. Kelly, The Basic Concepts of Enriched Category Theory, London Mathematical Society Lecture Notes Series, 64, Cambridge University Press, Cambridge, (1983). MR 84e:18001

[37] J. T. Lisica and S. Mardešić, Coherent prohomotopy and strong shape theory, Glasnik Mat., 19, (1984), 335-399. MR 87h:55005

[38] S. Mac Lane, Categories for the working mathematician, Graduate Texts in Math., Vol. 5, Springer-Verlag, Berlin, Heidelberg, New York, (1971). MR 50:7275

[39] S. Mardešić and J.Segal, Shape Theory, the Inverse Systems Approach, North-Holland Mathematical Library vol 26, North-Holland, Amsterdam, (1982). MR 84b:55020

[40] J. P. May, Classifying spaces and fibrations, Memoirs Amer. Math. Soc., Vol. 155, (1975). MR 51:6806

[41] J.-P. Meyer, Bar and cobar constructions, J. Pure Applied Alg., 33, (1984), 163-207. MR 86g: 18010

[42] J.-P. Meyer, Mappings of bar constructions, Israel J. Math., 48, (1984), 331-339. MR 86a: 18011

[43] J. E. Roberts, Mathematical aspects of local cohomology, in Proceedings of the Colloquium on Operator Algebras and their Application to Mathematical Physics, Marseille, 1977. MR 81e: 18017

[44] C. A. Robinson, Torsion Products as Homotopy Groups, J. Pure Applied Algebra, 21, (1981), 167-182. MR 83f: 18015

[45] D. G. Quillen, Homotopical Algebra, Lecture Notes in Mathematics, vol. 43, Springer-Verlag, Berlin, Heidelberg, New York, (1967). MR 36:6480

[46] G. Segal, Categories and cohomology theories, Topology, 13, (1974), 293-312. MR 50:5782

[47] E. H. Spanier, Algebraic Topology, McGraw-Hill, New York, (1966). MR 35:1007 
[48] R.Street, The algebra of oriented simplexes, J. Pure Appl. Algebra 49 (1987) 283-335; also, Higher Dimensional Nerves, Notes, McGill 16 and 23 April 1985, and Weak n-categories, Notes of lectures at Bangor, June 1993. MR 89a:18019

[49] M. Tierney and W. Vogel, Simplicial Resolutions and Derived Functors, Math. Z. 111, (1969), 1-14. MR 40:2733

[50] F. Ulmer, Kan Extensions, Cotriples and André (Co)homology, in Category Theory, Homology Theory and their Applications, II, Lecture Notes in Mathematics, No. 92, (1969), 278-308. MR 41:1840b

[51] D. Verity, Nerves of n-categories, Notes of lectures at Bangor, 1993.

[52] R. M. Vogt, Homotopy Limits and Colimits, Math. Z., 134, (1973), 11-52. MR 48:9709

Faculté de Mathématiques et d’Informatique, Université de Picardie - Jules Verne, 33 Rue Saint Leu, 80039 Amiens Cédex 1, France

E-mail address: cordier@mathinfo.u-picardie.fr

School of Mathematics, University of Wales, Bangor, Dean Street, Bangor, GWynedd, LL57 1UT, Wales, United Kingdom

E-mail address: t.porter@bangor.ac.uk 